\title{
Behavior of 5-amino-3-methylisoxazole in multicomponent heterocyclizations with carbonyl compounds under thermal heating and non-classical conditions
}

\author{
Elena A. Muravyova, ${ }^{a}$ Volodymyr V. Tkachenko, ${ }^{\text {a,b }}$ Sergey M. Desenko, \\ Yulia V. Sen'ko, ${ }^{a}$ Thomas J. J. Müller, ${ }^{c}$ Elena V. Vashchenko, \\ and Valentin A. Chebanov ${ }^{\mathrm{a}, \mathrm{b}, \mathrm{c}_{*}}$ \\ "Division of Chemistry of Functional Materials, SSI "Institute for Single Crystals" \\ NAS of Ukraine, Lenin Ave. 60, 61001 Kharkiv, Ukraine \\ ${ }^{b}$ Faculty of Chemistry, V.N. Karazin Kharkiv National University, Svobody sq., 4, \\ 61077 Kharkiv, Ukraine \\ ${ }^{c}$ Institut für Organische Chemie und Makromolekulare Chemie, Heinrich-Heine-Universität \\ Düsseldorf, Universitätsstraße 1, D-40225, Düsseldorf, Germany \\ E-mail:chebanov@isc.kharkov.com
}

\begin{abstract}
Three-component heterocyclizations of 5-amino-3-methylisoxazole, cyclohexanedione derivatives, and aromatic aldehydes, including salicylic aldehydes, are studied under conventional thermal heating, microwave irradiation and ultrasonication. A dependence of the direction of the reaction on the structure of the aldehyde and the reaction conditions was found, which allowed selective synthesis of 6,7,8,9-tetrahydroisoxazolo[5,4- $b$ ]quinolin-5(4H)-ones and 2,3,4,9-tetrahydro-1H-xanthen-1-ones. Key stages of the reaction mechanisms are discussed.
\end{abstract}

Keywords: Multicomponent reaction, heterocycles, 5-amino-3-methylisoxazole, microwaveassisted synthesis, ultrasound-assisted synthesis

\section{Introduction}

One of the main challenges of topical chemistry is the efficient design and synthesis of biologically active molecules. The discovery of high-throughput screening has tremendously increased the demand for new testing compounds and, therefore, multicomponent reactions (MCRs) became increasingly useful tools for the synthesis of biologically active compounds. These reactions enable multi-step syntheses to be conducted in a one-pot fashion to obtain a variety of invaluable products. Moreover, MCRs can dramatically reduce the generation of chemical waste and reduce the cost of the starting materials. In the past several years various 
multicomponent reactions that can provide easy and rapid accesses to useful functionalized multiple ring structures have been developed.

Isoxazole is an important heterocyclic unit, which has been widely used as a key buildingblock. Its derivatives are endowed with many pharmacological properties, such as hypoglycemic, analgesic, anti-inflammatory, antibacterial, anti-HIV, and anticancer activity, ${ }^{1-3}$ as well as useful activities in conditions like schizophrenia, hypertension, and Alzheimer's disease. ${ }^{4-6}$ Among the isoxazole derivatives, isoxazolopyridines have evoked interest and concern because they showed muscle relaxant, anticonvulsant and CNS depressant activities. ${ }^{7}$ To the best of our knowledge, multicomponent reactions involving isoxazole core and carbonyl compounds have been insufficiently studied and there have been only a few publications on the subject. For instance, Shi et $a l^{8}{ }^{8}$ studied the reaction of the aldehydes, mercaptoacetic acid and 5-aminoisoxazole in order to develop diversity-oriented synthesis of novel 1,4-thiazepan-3-ones derivatives embedded with the isoxazole motif. In another publication ${ }^{9}$ some new 4-aza-2,3-didehydropodophyllotoxin congeners were synthesized by applying a multicomponent route involving the condensation of substituted aminoisoxazole, tetronic acid and aromatic aldehydes in refluxing ethanol.

$\mathrm{Tu}$ and co-authors ${ }^{10}$ studied similar microwave-assisted three-component reaction of 5-amino-3-methylisoxazole and aldehydes with several active methylene compounds, such as tetronic acid, Meldrum's acid, 1,3-indanedione and 1,3-cyclohexanediones. There was shown a superior advantage of water as reaction medium and microwave irradiation to promote these treatments. It is worthy to note that, for some reasons which remained obscure, the reaction involving dimedone gave isoxazolo[5,4-b]pyridine while in the case of 1,3-cyclohexanedione the final compound was its dihydro derivative (Scheme 1).

Condensation with Meldrum's acid via ring-opening and release of acetone and carbon dioxide led to the formation of 4.7-dihydroisoxazolo[5,4- $b]$ pyridine- $6(5 H)$-one. ${ }^{10}$ However, in another article $^{11}$ the same group reported that the reaction of Meldrum's acid with 5-amino-3methylisoxazole and aldehydes under analogous conditions yielded spiro[pyrazolo[1,3]dioxanopyridine]-4,6-diones instead of isoxazolopyridinones (Scheme 1).

In addition, the three-component reaction of 5-amino-3-methylisoxazole with 2-hydroxy-1,4naphthoquinone and aromatic aldehydes leading to benzo[ $h]$ isoxazolo[5,4- $b]$ quinoline-5,6diones was studied by Tu et al. in a subsequent article. ${ }^{12}$

Thus, it is obvious that published results provide insufficient and sometimes conflicting information on multicomponent heterocyclizations of 5-amino-3-methylisoxazole and, therefore, such processes are challenging objects of detailed study. In the present article we continue developing our concept of tuning the selectivity of MCRs ${ }^{13-22}$ (for reviews see refs 13-15). and disclose our recent results in the field of study of MCRs involving 5-amino-3-methylisoxazole, 1,3-cyclohexanediones, and aromatic aldehydes including salicylaldehydes using different reaction conditions (temperature regimes, solvents, catalysts and modes of activation). 


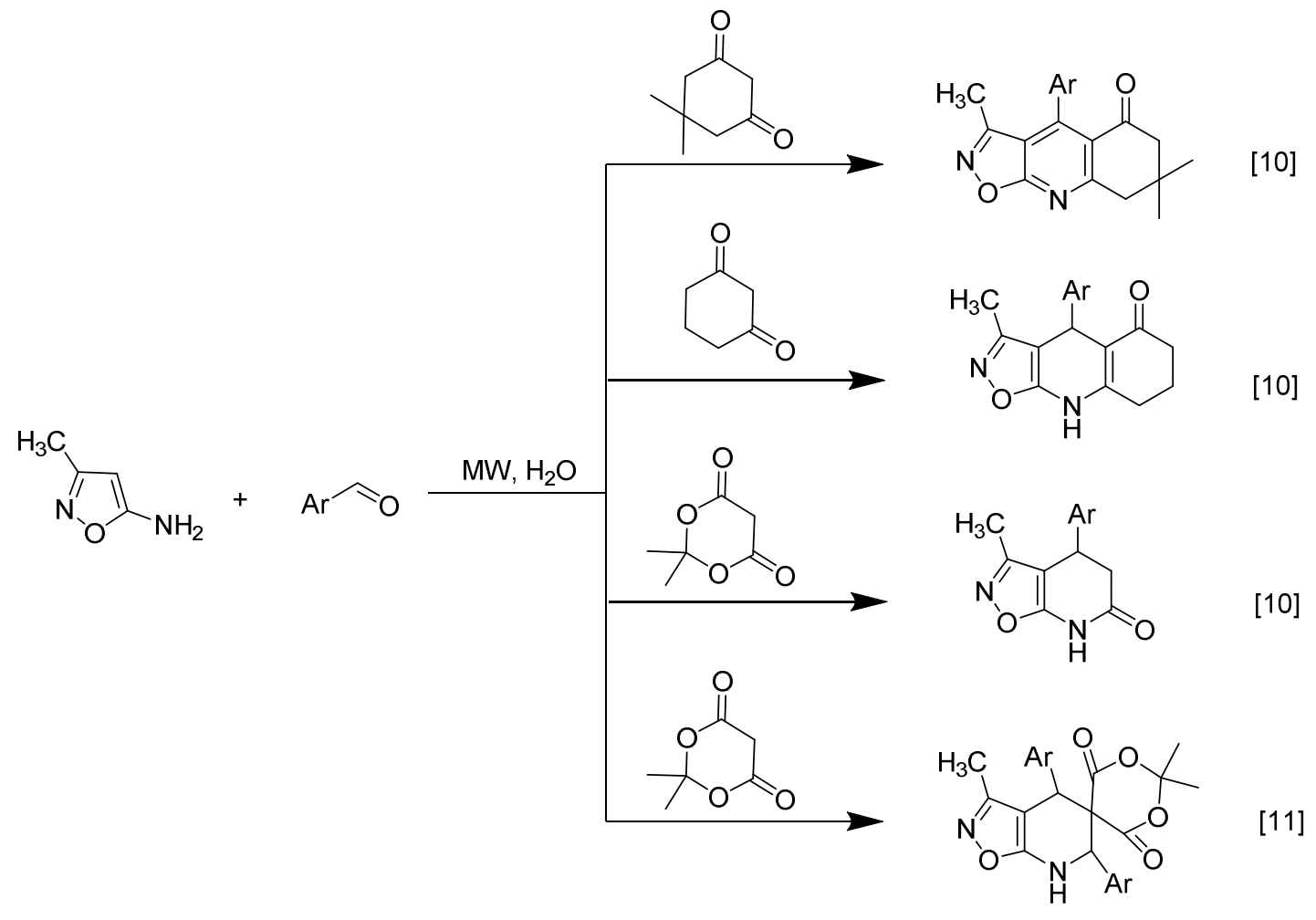

Scheme 1. Some known multicomponent reactions involving 5-amino-3-methylisoxazole

\section{Results and Discussion}

The MCR between 5-amino-3-methylisoxazole (1), 4-bromobenzaldehyde (2c), and dimedone (3b) was chosen in order to search for optimal conditions (solvent, activation method, reaction temperature and time). Among the solvents tested (water, different alcohols, DMF, HOAc) water, ethanol and DMF were selected for the model reaction due to better preliminary results.

Ultrasonic-promoted (US) procedures gave the worst results with respect to yields and purity of 4-(4-bromophenyl)-3,7,7-trimethyl-6,7,8,9-tetrahydroisoxazolo[5,4-b]quinolin-5(4H)-one (41) which was isolated as sole reaction product in the MCR studied (Table 1). The situation was better in the case of conventional heating $(\Delta)$ of the starting materials in these solvents - yields of the target heterocycle $4 \mathrm{l}$ lay in the range from $50 \%$ for EtOH to $75 \%$ for DMF while their purity was about 95\% (NMR control). However, irrespective of the solvent type, the best yields of the compound 4l ( $90 \%)$ with high purity were observed when the MCR was carried out under microwave irradiation (MW) at $120{ }^{\circ} \mathrm{C}$. At lower temperatures the yields of compound $4 \mathbf{l}$ decreased sufficiently while carrying out the reaction at temperatures higher than $140{ }^{\circ} \mathrm{C}$ gave the final heterocycles in unsatisfactory purity or even led to decomposition.

Thus, the above-mentioned results for the model three-component reaction allowed us to choose refluxing the starting materials in DMF or the microwave-assisted reaction in ethanol as procedures for the further study. 
It was found that MCRs between 5-amino-3-methylisoxazole (1), aldehydes 2a-i, and cyclic $\beta$-diketones 3a-c in boiling DMF led to the formation of 4-aryl-6,7,8,9-tetrahydroisoxazolo[5,4-b]quinolin-5(4H)-ones $\mathbf{4 a - z}$ in $55-91 \%$ yields (Scheme 2, Table 2). It should be noted that the microwave-assisted procedure in some cases gave the desired targets in higher yields than conventional heating as it was observed for model reactions (entries 3, 11, 12, 14. 19, 20 and 24), however sometimes the yields were practically the same under both activation methods (entries $1,2,4,6,7,13,15$ and 20).

Table 1. Optimization of the conditions of three-component reaction between 5-amino-3-methylisoxazole, 4-bromobenzaldehyde and 5,5-dimethylcyclohexane-1.3-dione

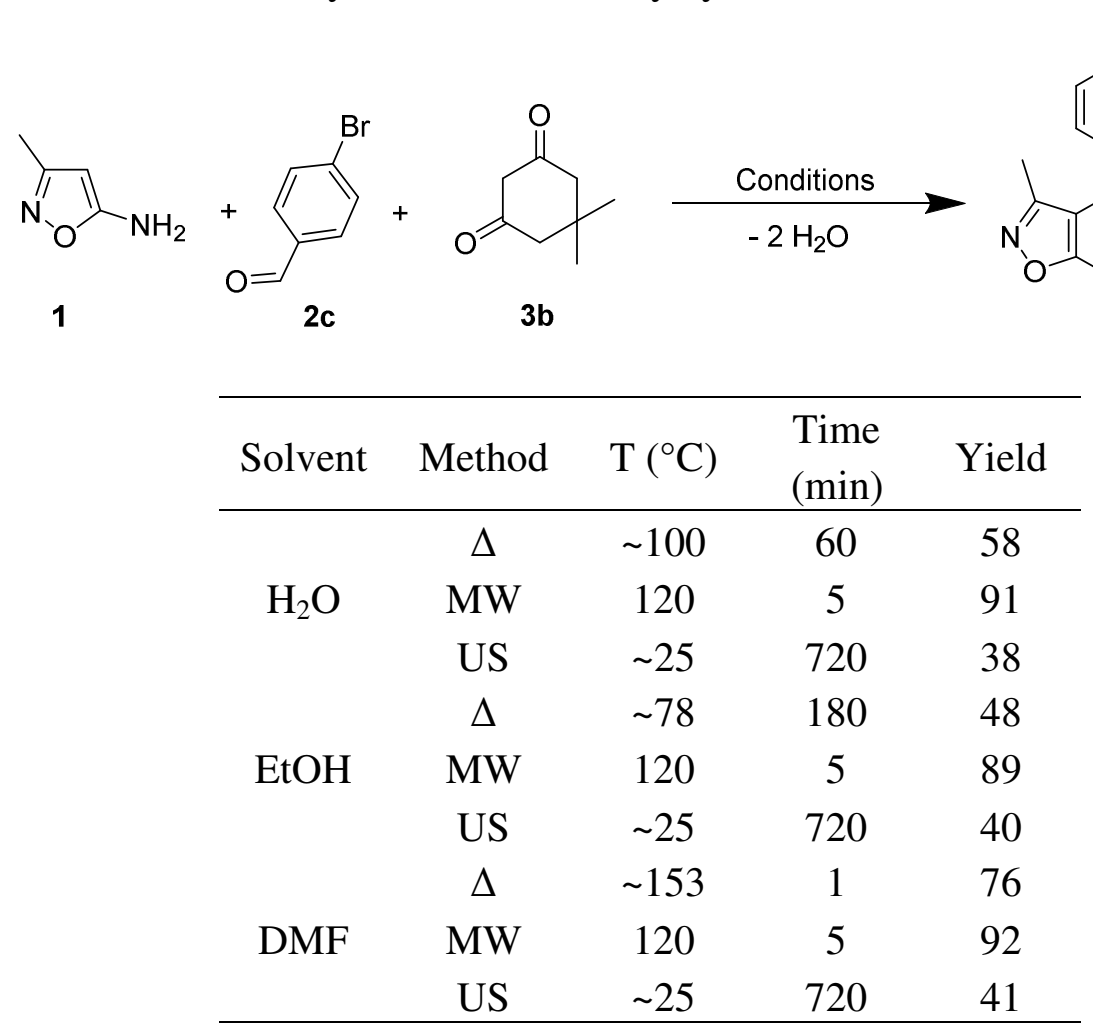

When 5-(4-chlorophenyl)cyclohexane-1,3-dione (3c) was used the final compounds contained two chiral centers and in several cases the formation of mixtures of diastereomers with different ratios were isolated (entries 19, 20, 22-25, Table 2). 

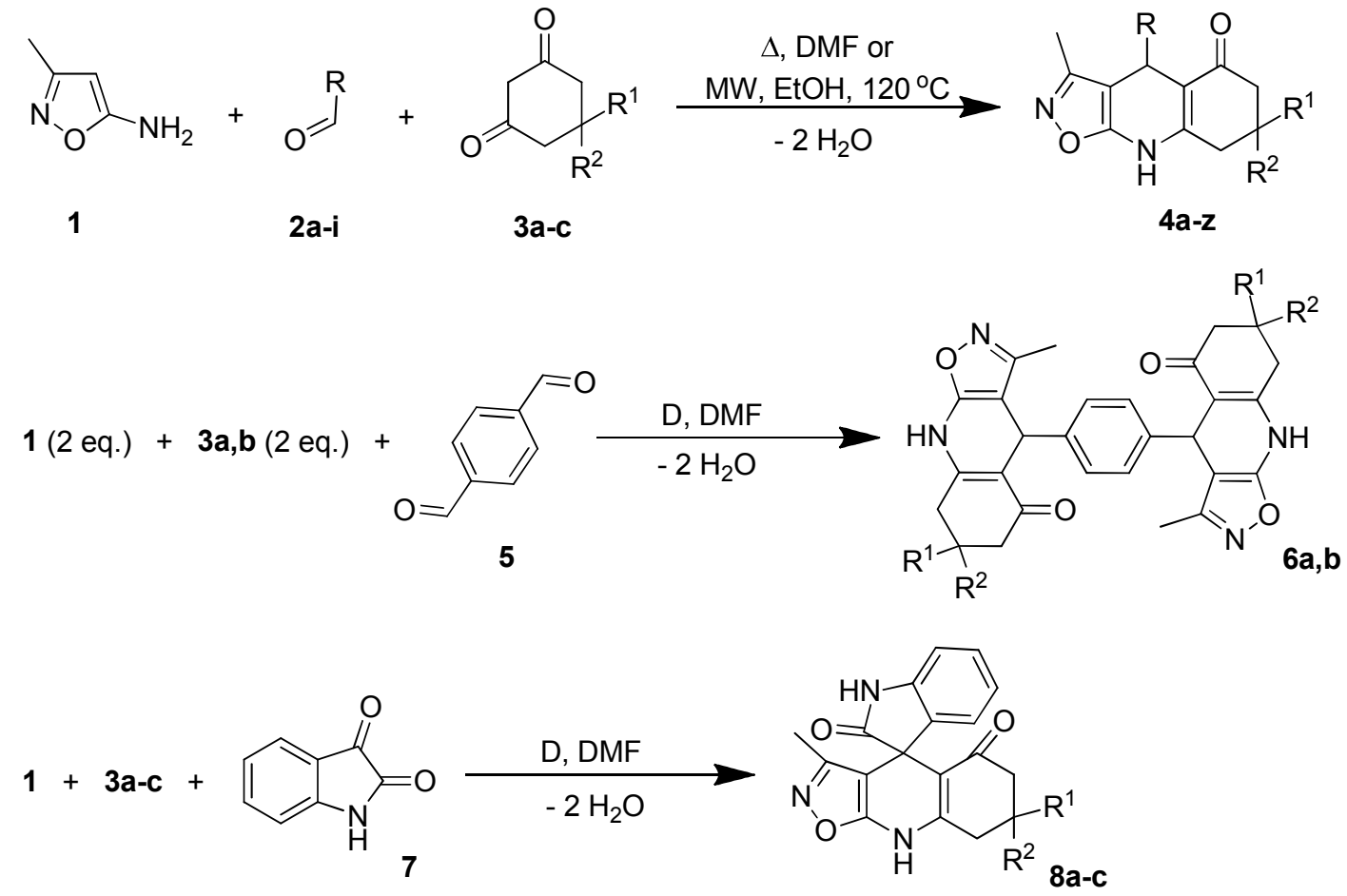

Scheme 2. Multicomponent microwave-assisted synthesis of products $\mathbf{4 a - z}, \mathbf{6 a}, \mathbf{b}$ and $\mathbf{8 a - c}$.

Table 2. Three-component synthesis of compounds $4 \mathbf{a}-\mathbf{z}, \mathbf{6 a - b}$ and $\mathbf{8 a - c}$

\begin{tabular}{|c|c|c|c|c|c|c|c|c|}
\hline \multirow{4}{*}{$\begin{array}{l}\text { En- } \\
\text { try }\end{array}$} & \multicolumn{5}{|c|}{ Building-blocks } & \multirow{2}{*}{\multicolumn{3}{|c|}{ Reaction product }} \\
\hline & \multicolumn{2}{|c|}{ Carbonyl compound } & \multicolumn{3}{|c|}{$\beta$-Diketone } & & & \\
\hline & \multirow{2}{*}{\multicolumn{2}{|c|}{$\mathrm{R}$}} & & \multirow{2}{*}{$\mathrm{R}^{1}$} & \multirow{2}{*}{$\mathrm{R}^{2}$} & & \multicolumn{2}{|c|}{ Yield, \% } \\
\hline & & & & & & & $\Delta$ & MW \\
\hline 1 & $2 \mathbf{a}$ & $\mathrm{C}_{6} \mathrm{H}_{5}$ & $\mathbf{3 a}$ & $\mathrm{H}$ & $\mathrm{H}$ & $4 \mathbf{a}$ & 65 & 68 \\
\hline 2 & $2 b$ & $4-\mathrm{ClC}_{6} \mathrm{H}_{4}$ & 3a & $\mathrm{H}$ & $\mathrm{H}$ & $4 b$ & 75 & 74 \\
\hline 3 & $2 c$ & $4-\mathrm{BrC}_{6} \mathrm{H}_{4}$ & 3a & $\mathrm{H}$ & $\mathrm{H}$ & $4 c$ & 75 & 90 \\
\hline 4 & 2d & $4-\mathrm{CH}_{3} \mathrm{C}_{6} \mathrm{H}_{4}$ & 3a & $\mathrm{H}$ & $\mathrm{H}$ & $4 d$ & 75 & 76 \\
\hline 5 & $2 e$ & $4-\mathrm{CH}_{3} \mathrm{OC}_{6} \mathrm{H}_{4}$ & 3a & $\mathrm{H}$ & $\mathrm{H}$ & $4 e$ & 85 & - \\
\hline 6 & $2 f$ & $4-\mathrm{O}_{2} \mathrm{NC}_{6} \mathrm{H}_{4}$ & 3a & $\mathrm{H}$ & $\mathrm{H}$ & $4 f$ & 60 & 62 \\
\hline 7 & $2 g$ & $4-\mathrm{CH}_{3} \mathrm{OCOC}_{6} \mathrm{H}_{4}$ & 3a & $\mathrm{H}$ & $\mathrm{H}$ & $4 g$ & 60 & 62 \\
\hline 8 & $2 h$ & $4-\left(\mathrm{CH}_{3}\right)_{2} \mathrm{NC}_{6} \mathrm{H}_{4}$ & 3a & $\mathrm{H}$ & $\mathrm{H}$ & $4 h$ & 65 & - \\
\hline 9 & $2 \mathbf{i}$ & $\left(\mathrm{CH}_{3}\right)_{2} \mathrm{CH}$ & 3a & $\mathrm{H}$ & $\mathrm{H}$ & $4 \mathbf{i}$ & 75 & - \\
\hline 10 & $\mathbf{2 a}$ & $\mathrm{C}_{6} \mathrm{H}_{5}$ & 3a & $\mathrm{CH}_{3}$ & $\mathrm{CH}_{3}$ & $4 \mathbf{j}$ & 75 & - \\
\hline 11 & $2 \mathbf{b}$ & $4-\mathrm{ClC}_{6} \mathrm{H}_{4}$ & $3 b$ & $\mathrm{CH}_{3}$ & $\mathrm{CH}_{3}$ & $4 k$ & 65 & 80 \\
\hline 12 & $2 \mathrm{c}$ & $4-\mathrm{BrC}_{6} \mathrm{H}_{4}$ & $3 b$ & $\mathrm{CH}_{3}$ & $\mathrm{CH}_{3}$ & 41 & 76 & 92 \\
\hline 13 & $2 d$ & $4-\mathrm{CH}_{3} \mathrm{C}_{6} \mathrm{H}_{4}$ & $3 b$ & $\mathrm{CH}_{3}$ & $\mathrm{CH}_{3}$ & $4 m$ & 60 & 62 \\
\hline 14 & $2 \mathbf{e}$ & $4-\mathrm{CH}_{3} \mathrm{OC}_{6} \mathrm{H}_{4}$ & $3 b$ & $\mathrm{CH}_{3}$ & $\mathrm{CH}_{3}$ & $4 n$ & 71 & 90 \\
\hline
\end{tabular}


Table 2. Continued

\begin{tabular}{|c|c|c|c|c|c|c|c|c|}
\hline \multirow{4}{*}{$\begin{array}{l}\text { En- } \\
\text { try }\end{array}$} & \multicolumn{5}{|c|}{ Building-blocks } & \multirow{2}{*}{\multicolumn{3}{|c|}{ Reaction product }} \\
\hline & \multicolumn{2}{|c|}{ Carbonyl compound } & \multicolumn{3}{|c|}{$\beta$-Diketone } & & & \\
\hline & \multirow{2}{*}{\multicolumn{2}{|c|}{$\mathrm{R}$}} & \multirow{2}{*}{\multicolumn{2}{|c|}{$\mathrm{R}^{1}$}} & \multirow{2}{*}{$\mathrm{R}^{2}$} & & \multicolumn{2}{|c|}{ Yield, \% } \\
\hline & & & & & & & $\Delta$ & MW \\
\hline 15 & $2 f$ & $4-\mathrm{NO}_{2} \mathrm{C}_{6} \mathrm{H}_{4}$ & $\mathbf{3 b}$ & $\mathrm{CH}_{3}$ & $\mathrm{CH}_{3}$ & 40 & 55 & 58 \\
\hline 16 & $2 \mathrm{~g}$ & $4-\mathrm{CH}_{3} \mathrm{OCOC}_{6} \mathrm{H}_{4}$ & $\mathbf{3 b}$ & $\mathrm{CH}_{3}$ & $\mathrm{CH}_{3}$ & $4 p$ & 90 & - \\
\hline 17 & $2 \mathbf{h}$ & 4- $\left(\mathrm{CH}_{3}\right)_{2} \mathrm{NC}_{6} \mathrm{H}_{4}$ & $\mathbf{3 b}$ & $\mathrm{CH}_{3}$ & $\mathrm{CH}_{3}$ & $4 q$ & 90 & - \\
\hline 18 & $2 \mathbf{i}$ & $\left(\mathrm{CH}_{3}\right)_{2} \mathrm{CH}$ & $\mathbf{3 b}$ & $\mathrm{CH}_{3}$ & $\mathrm{CH}_{3}$ & $4 r$ & 85 & - \\
\hline 19 & $\mathbf{2 a}$ & $\mathrm{C}_{6} \mathrm{H}_{5}$ & $3 c$ & $\mathrm{H}$ & $4-\mathrm{ClC}_{6} \mathrm{H}_{4}$ & $4 s$ & $55(50 / 50)^{\mathrm{a}}$ & $\begin{array}{c}80 \\
(50 / 50)^{\mathrm{a}}\end{array}$ \\
\hline 20 & $2 \mathbf{b}$ & $4-\mathrm{ClC}_{6} \mathrm{H}_{4}$ & $3 c$ & $\mathrm{H}$ & $4-\mathrm{ClC}_{6} \mathrm{H}_{4}$ & $4 \mathbf{t}$ & $65(50 / 50)^{\mathrm{a}}$ & $\begin{array}{c}67 \\
(50 / 50)^{\mathrm{a}}\end{array}$ \\
\hline 21 & $2 c$ & $4-\mathrm{BrC}_{6} \mathrm{H}_{4}$ & $3 c$ & $\mathrm{H}$ & $4-\mathrm{ClC}_{6} \mathrm{H}_{4}$ & $4 \mathbf{u}$ & $90(100 / 0)^{\mathrm{a}}$ & - \\
\hline 22 & 2d & 4- $\mathrm{CH}_{3} \mathrm{C}_{6} \mathrm{H}_{4}$ & $3 c$ & $\mathrm{H}$ & $4-\mathrm{ClC}_{6} \mathrm{H}_{4}$ & $4 v$ & $90(95 / 5)^{\mathrm{a}}$ & - \\
\hline 23 & $2 \mathbf{e}$ & $4-\mathrm{CH}_{3} \mathrm{OC}_{6} \mathrm{H}_{4}$ & $3 c$ & $\mathrm{H}$ & 4- $\mathrm{ClC}_{6} \mathrm{H}_{4}$ & $4 w$ & $91(95 / 5)^{\mathrm{a}}$ & - \\
\hline 24 & $2 f$ & $4-\mathrm{NO}_{2} \mathrm{C}_{6} \mathrm{H}_{4}$ & $3 c$ & $\mathrm{H}$ & $4-\mathrm{ClC}_{6} \mathrm{H}_{4}$ & $4 x$ & $58(50 / 50)^{\mathrm{a}}$ & $\begin{array}{c}64 \\
(50 / 50)^{\mathrm{a}}\end{array}$ \\
\hline 25 & $2 \mathrm{~g}$ & 4- $\mathrm{CH}_{3} \mathrm{OCOC}_{6} \mathrm{H}_{4}$ & $3 c$ & $\mathrm{H}$ & $4-\mathrm{ClC}_{6} \mathrm{H}_{4}$ & $4 y$ & $85(90 / 10)^{\mathrm{a}}$ & - \\
\hline 26 & $2 h$ & $4-\left(\mathrm{CH}_{3}\right)_{2} \mathrm{NC}_{6} \mathrm{H}_{4}$ & $3 c$ & $\mathrm{H}$ & $4-\mathrm{ClC}_{6} \mathrm{H}_{4}$ & $4 z$ & $90(100 / 0)^{\mathrm{a}}$ & - \\
\hline 27 & 5 & - & $\mathbf{3 a}$ & $\mathrm{H}$ & $\mathrm{H}$ & $6 \mathbf{a}$ & 56 & - \\
\hline 28 & 5 & - & $\mathbf{3 b}$ & $\mathrm{CH}_{3}$ & $\mathrm{CH}_{3}$ & $6 \mathbf{b}$ & 60 & - \\
\hline 29 & 7 & - & $\mathbf{3 a}$ & $\mathrm{H}$ & $\mathrm{H}$ & $8 \mathbf{a}$ & 75 & - \\
\hline 30 & 7 & - & $3 \mathbf{b}$ & $\mathrm{CH}_{3}$ & $\mathrm{CH}_{3}$ & $8 b$ & 72 & - \\
\hline 31 & 7 & - & $3 \mathrm{c}$ & $\mathrm{H}$ & $4-\mathrm{ClC}_{6} \mathrm{H}_{4}$ & $8 c$ & 65 & - \\
\hline
\end{tabular}

${ }^{\text {a }}$ ratio $(\%)$ of diastereomers according to ${ }^{1} \mathrm{H}$ NMR data.

In the case of terephthalic aldehyde (5) in the MCR were involved both its carbonyl groups which allowed to isolate compounds $\mathbf{6 a}$ and $\mathbf{6 b}$ in 56-60\% yields (amine $\mathbf{1}$ and diketones 3a,b were used in the reaction in 2-fold excess). Isatine was also introduced in this three-component heterocyclization instead of an aldehyde to give spiro-compounds 8a-c in $65-75 \%$ yields (Scheme 2, Table 2). Despite the presence of two chiral centers in the heterocycle 8c no mixture of diastereomers was obtained in this case.

Application of $o$-salicylic aldehyde in MCRs may complicate their proceeding due to different intramolecular cyclizations involving the $\mathrm{OH}$-group. ${ }^{23-26}$ For instance, Gorobets et al. ${ }^{23}$ described a Biginelli-type three-component reaction between 3-amino-1,2,4-triazole, several carbonyl containing $\mathrm{CH}$-acids and aldehydes. They showed that in the case of salicylic aldehyde depending on conditions applied the treatment yielded either 4,5,6,7-tetrahydro[1,2,4]- 
triazolo[1,5-a]pyrimidines or 11,12-dihydro-5,11-methano[1,2,4]triazolo[1,5-c][1,3,5]benzoxadiazocines. On the other hand, Světlík and Kettmann ${ }^{24}$ in a very similar reaction observed the formation of spiro-compounds instead of bridged heterocycles. Another direction for such reaction giving chromeno[4,3-d]pyrazolo[3,4-b]pyridines was described by Frolova et al. ${ }^{25}$ and Světlík et al. ${ }^{26}$

In our case the three-component reaction of 5-amino-3-methylisoxazole (1), cyclic 1,3diketones 3a-c and salicylic aldehydes 9a-e in DMF or $\mathrm{H}_{2} \mathrm{O}$ under conventional thermal heating or under microwave irradiation yielded the mixture of 6,7,8,9-tetrahydroisoxazolo[5,4- $b$ ]quinolin-5(4H)-ones 10a-o, 2,3,4,9-tetrahydro- $H$-xanthen-1-ones 11a-o and 12a-o in 1:3:1 ratio (Scheme 3, Table 3). Heterocycles obtained were separated by crystallization - compounds 10ao were precipitated from acetone while compounds 11a-o and 12a-o from ethyl acetate. It should be noted that mode of activation had no influence on the ratio of substances in the mixture.

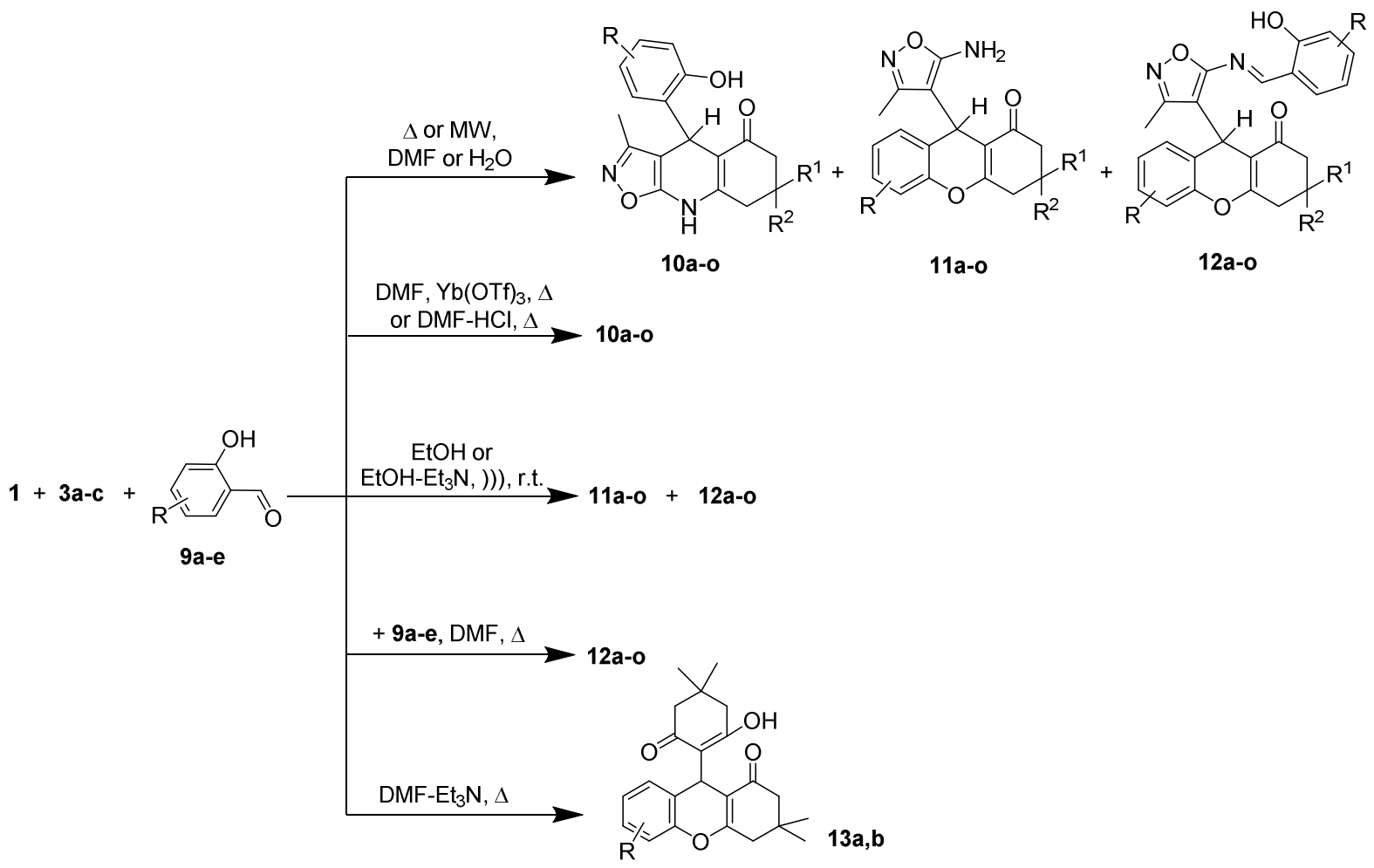

Scheme 3. Multicomponent reactions of 5-amino-3-methylisoxazole (1), cyclic 1,3-diketones 3ac and salicylaldehydes $\mathbf{9 a - e . ~}$

By using pure ethanol or its mixture with a catalytic amount of $\mathrm{Et}_{3} \mathrm{~N}$ the formation of quinolinones 10 was avoided and only compounds 11a-o and 12a-o were isolated in individual state by crystallization from ethyl acetate. In the case of basic catalysis the mixtures were enriched with compound 11 (75\%) while the ratio of $\mathbf{1 1}$ and $\mathbf{1 2}$ under neutral conditions was 1:1. It can be explained by influence of acidity of the reaction medium on the formation of 
azomethine since basic additives did not promote the reaction leading to such imines in contrast to neutral or acidic conditions.

It is noteworthy that the MCR of the starting compounds in boiling in DMF in the presence of catalytic amounts of $\mathrm{Et}_{3} \mathrm{~N}$ yielded xanthen-1-ones 13a-c formed without participation of the aminoisoxazole building-block.

Addition to the reaction mixture of a second equivalent of aldehyde allowed directing the MCR in boiling DMF or EtOH towards the exclusive formation of azomethines 12a-o while the selective procedure for the synthesis of compounds 11a-o was not elaborated.

Isoxazolo[5,4- $b$ ]quinolinones 10a-o were selectively obtained when 5-amino-3-methylisoxazole 1, diketones 3a-c, and salicylic aldehydes 9a-e reacted in boiling DMF in the presence of catalytic amounts of $\mathrm{HCl}$ or $\mathrm{Yb}(\mathrm{OTf})_{3}(5 \mathrm{~mol} \%)$. The yield of this reaction was moderate [45$50 \%$ for $\left.\mathrm{Yb}(\mathrm{OTf})_{3}\right]$, however, this result gives a strong background to be elaborated in a future highly effective and selective procedure for the synthesis of compounds like $\mathbf{1 0}$ with application of water-stable Lewis acids.

Additionally it should be noted that oxidation of compounds $\mathbf{4 f}, \mathbf{k}-\mathbf{m}, \mathbf{p}, \mathbf{u}, \mathbf{w}$ with NBS afforded isoxazolo[5,4- $b$ ]quinolin-5(4H)-ones 14f,k-m,p,u,w (Scheme 4). Furthermore, the pyridine $\mathrm{NH}$ in heterocycles 4 was easily alkylated with alkyl halides in DMF $-\mathrm{K}_{2} \mathrm{CO}_{3}$ or DMF - $\mathrm{KOH}$ to give compounds 15a-e in $42-65 \%$ yields (Scheme 4).

Most likely the three-component reaction of 5-amino-3-methylisoxazole, aldehydes, and derivatives of 1,3-cyclohexanedione pass via formation of Michael adduct $\mathbf{I}$ (Scheme 5) and its further cyclization due to the nucleophilic attack at the carbonyl by $\mathrm{NH}_{2}$-group giving up isoxazoloquinolinones $4,6,8$ or 10 .

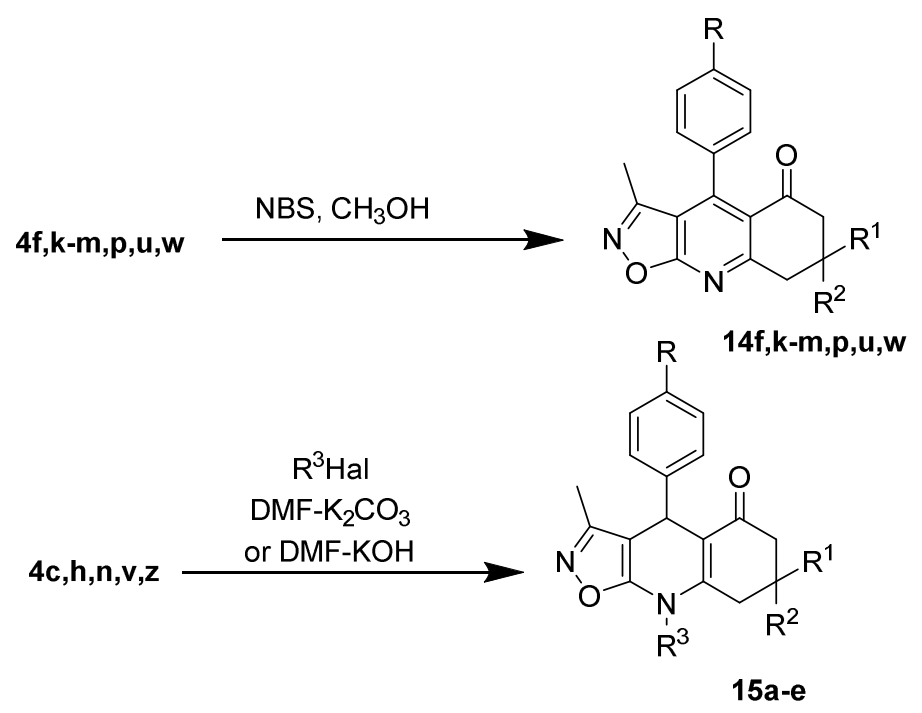

15a-e: $a \mathrm{R}=4-\mathrm{BrC}_{6} \mathrm{H}_{4}, \mathrm{R}^{1}=\mathrm{H}, \mathrm{R}^{2}=\mathrm{H}, \mathrm{R}^{3}=\mathrm{C}_{2} \mathrm{H}_{5} ; \mathrm{b} \mathrm{R}=4-\mathrm{N}\left(\mathrm{CH}_{3}\right)_{2} \mathrm{C}_{6} \mathrm{H}_{4}, \mathrm{R}^{1}=\mathrm{H}, \mathrm{R}^{2}=\mathrm{H}, \mathrm{R}^{3}=\mathrm{CH}_{2} \mathrm{C}_{6} \mathrm{H}_{5}$; $c \mathrm{R}=4-\mathrm{CH}_{3} \mathrm{OC}_{6} \mathrm{H}_{4}, \mathrm{R}^{1}=\mathrm{CH}_{3}, \mathrm{R}^{2}=\mathrm{CH}_{3}, \mathrm{R}^{3}=\mathrm{CH}_{2} \mathrm{C}_{6} \mathrm{H}_{5} ; \mathrm{d} \mathrm{R}=4-\mathrm{CH}_{3} \mathrm{C}_{6} \mathrm{H}_{4}, \mathrm{R}^{1}=\mathrm{H}, \mathrm{R}^{2}=4-\mathrm{ClC}_{6} \mathrm{H}_{4}, \mathrm{R}^{3}=\mathrm{C}_{2} \mathrm{H}_{5}$; e R $=4-\mathrm{CH}_{3} \mathrm{C}_{6} \mathrm{H}_{4}, \mathrm{R}^{1}=\mathrm{H}, \mathrm{R}^{2}=4-\mathrm{ClC}_{6} \mathrm{H}_{4}, \mathrm{R}^{3}=\mathrm{CH}_{2} \mathrm{C}_{6} \mathrm{H}_{5} ; \mathrm{f} \mathrm{R}=4-\mathrm{N}\left(\mathrm{CH}_{3}\right)_{2} \mathrm{C}_{6} \mathrm{H}_{4}, \mathrm{R}^{1}=\mathrm{H}, \mathrm{R}^{2}=4-\mathrm{ClC}_{6} \mathrm{H}_{4}, \mathrm{R}^{3}=\mathrm{CH}_{2} \mathrm{C}_{6} \mathrm{H}_{5}$

Scheme 4. Oxidation and N-alkylation of heterocycles 4. 
However, in the case of salicylic aldehyde (intermediate I') there is an alternative $\mathrm{OH}$ nucleophilic reaction center which is able to take part in the heterocyclization with formation of the pyran ring (compounds 11 and 12) instead of the pyridine core. According to experimental data obtained when the salicylic aldehyde is used acidic catalysis with Brønsted or Lewis acids promotes the Hantzsch type of MCR, while a presence in the reaction mixture of $\mathrm{Et}_{3} \mathrm{~N}$ redirects the reaction towards the formation of xanthenones.

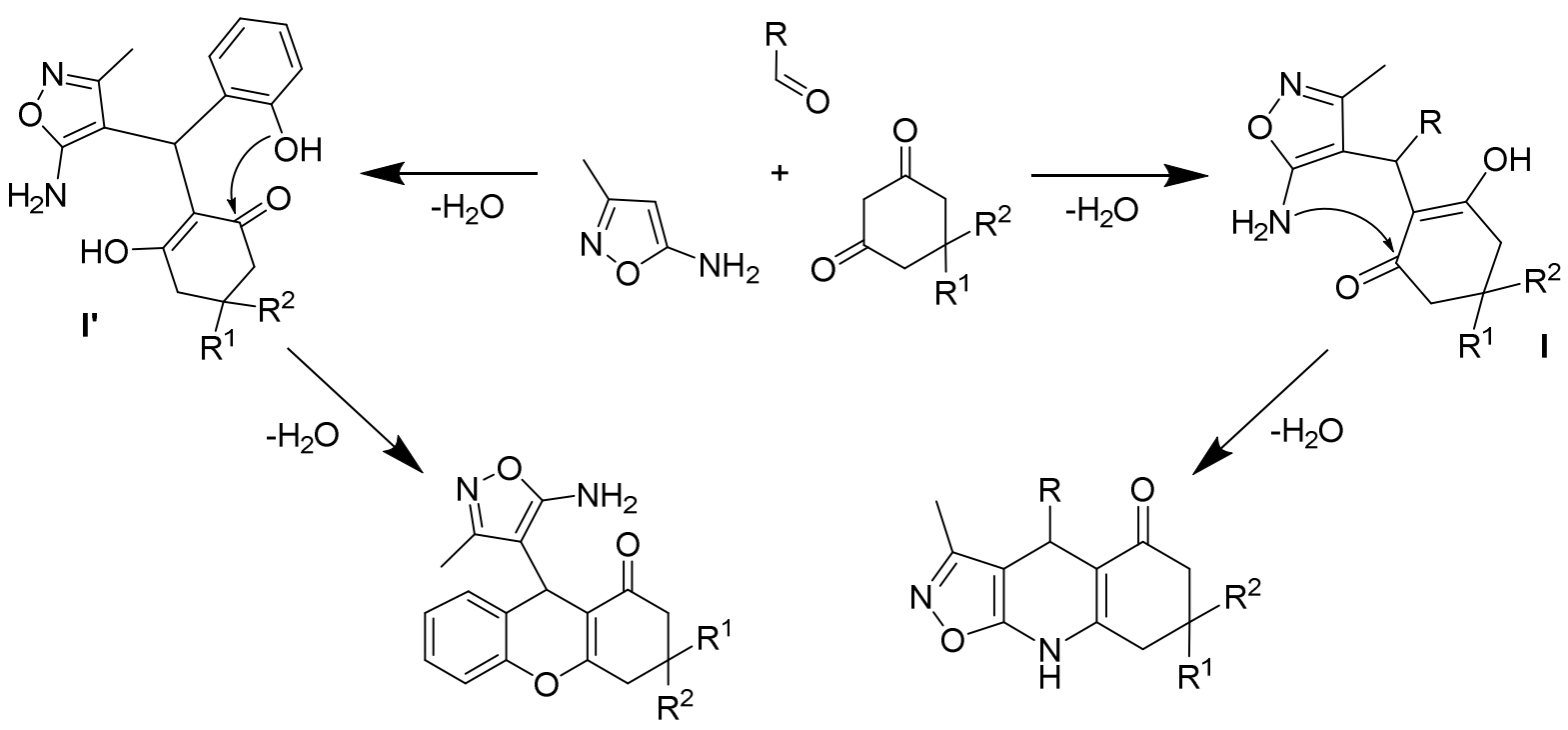

Scheme 5. Possible key-stages of the three-component reaction of 5-amino-3-methylisoxazole, aldehydes, and 1,3-cyclohexanediones.

Identification of all the compounds synthesized was made with help of elemental analysis, MS spectrometry, 1D and 2D NMR spectroscopy.

Thus, ${ }^{1} \mathrm{H}$ NMR spectra of compounds $4 \mathbf{a}-\mathbf{z}, \mathbf{6 a}, \mathbf{b}$ and 10a-o showed singlet of methine proton at $\delta$ 4.8-5.25 (disappearing in compounds 14 ), signals of $\mathrm{CH}_{2}$-groups of cycloalkenone fragment in the range of $\delta$ 2.0-2.9, a singlet of the $\mathrm{NH}$ proton near 10.4-11.0 ppm (disappearing in compounds 15) and signals of aryl group and terminal substituents at appropriate positions. The spectra of the compounds $\mathbf{4 s - z}$ additionally contain singlets of the $\mathrm{CH}$-group of cycloalkenone moiety and in the case of mixtures of diastereomers (see Table 2) - two sets of identical signals. The spectra of the compounds 8a-c exhibit no signal of methine group in pyridine ring but contain second broad singlet of NH-group of the isatin fragment. 
Table 3. Three-component synthesis of compounds 10a-o, 11a-o and 12a-o

\begin{tabular}{|c|c|c|c|c|c|c|c|}
\hline \multirow{3}{*}{$\begin{array}{l}\text { En- } \\
\text { try }\end{array}$} & \multicolumn{5}{|c|}{ Building-blocks } & \multirow{2}{*}{\multicolumn{2}{|c|}{ Reaction product }} \\
\hline & \multicolumn{2}{|c|}{ Salicylaldehyde } & & \multicolumn{2}{|c|}{$\beta$-Diketone } & & \\
\hline & & $\mathrm{R}$ & & $\mathrm{R}^{1}$ & $\mathrm{R}^{2}$ & & Yield, \% \\
\hline 1 & $9 \mathbf{a}$ & $\mathrm{H}$ & $3 \mathbf{a}$ & $\mathrm{H}$ & $\mathrm{H}$ & $10 \mathbf{a}$ & $45^{\mathrm{a}}$ \\
\hline 2 & $9 b$ & $5-\mathrm{Cl}$ & $\mathbf{3 a}$ & $\mathrm{H}$ & $\mathrm{H}$ & $10 b$ & $42^{\mathrm{a}}$ \\
\hline 3 & $9 c$ & $5-\mathrm{Br}$ & $\mathbf{3 a}$ & $\mathrm{H}$ & $\mathrm{H}$ & $10 \mathrm{c}$ & $38^{\mathrm{a}}$ \\
\hline 4 & 9d & $3-\mathrm{CH}_{3} \mathrm{O}$ & $\mathbf{3 a}$ & $\mathrm{H}$ & $\mathrm{H}$ & 10d & $40^{\mathrm{a}}$ \\
\hline 5 & $9 e$ & $5-\mathrm{NO}_{2}$ & $\mathbf{3 a}$ & $\mathrm{H}$ & $\mathrm{H}$ & $10 \mathrm{e}$ & $57^{\mathrm{a}}$ \\
\hline 6 & $9 \mathbf{a}$ & $\mathrm{H}$ & $3 b$ & $\mathrm{CH}_{3}$ & $\mathrm{CH}_{3}$ & $10 f$ & $42^{\mathrm{a}}$ \\
\hline 7 & $9 b$ & $5-\mathrm{Cl}$ & $\mathbf{3 b}$ & $\mathrm{CH}_{3}$ & $\mathrm{CH}_{3}$ & $10 \mathrm{~g}$ & $54^{\mathrm{a}}$ \\
\hline 8 & $9 c$ & $5-\mathrm{Br}$ & $3 b$ & $\mathrm{CH}_{3}$ & $\mathrm{CH}_{3}$ & 10h & $51^{\mathrm{a}}$ \\
\hline 9 & 9d & $3-\mathrm{CH}_{3} \mathrm{O}$ & $\mathbf{3 b}$ & $\mathrm{CH}_{3}$ & $\mathrm{CH}_{3}$ & $10 \mathrm{i}$ & $55^{\mathrm{a}}$ \\
\hline 10 & $9 e$ & $5-\mathrm{NO}_{2}$ & $3 b$ & $\mathrm{CH}_{3}$ & $\mathrm{CH}_{3}$ & $\mathbf{1 0 j}$ & $62^{\mathrm{a}}$ \\
\hline 11 & 9a & $\mathrm{H}$ & $3 \mathbf{c}$ & $\mathrm{H}$ & $4-\mathrm{ClC}_{6} \mathrm{H}_{4}$ & 10k & $38^{\mathrm{a}}(50 / 50)^{\mathrm{d}}$ \\
\hline 12 & $9 \mathrm{~b}$ & $5-\mathrm{Cl}$ & $3 \mathbf{c}$ & $\mathrm{H}$ & $4-\mathrm{ClC}_{6} \mathrm{H}_{4}$ & 101 & $45^{\mathrm{a}}(50 / 50)^{\mathrm{d}}$ \\
\hline 13 & $9 c$ & 5-Br & $3 c$ & $\mathrm{H}$ & $4-\mathrm{ClC}_{6} \mathrm{H}_{4}$ & $10 \mathrm{~m}$ & $51^{\mathrm{a}}(50 / 50)^{\mathrm{d}}$ \\
\hline 14 & $9 d$ & $3-\mathrm{CH}_{3} \mathrm{O}$ & $3 c$ & $\mathrm{H}$ & $4-\mathrm{ClC}_{6} \mathrm{H}_{4}$ & $10 n$ & $32^{\mathrm{a}}(50 / 50)^{\mathrm{d}}$ \\
\hline 15 & $9 e$ & $5-\mathrm{NO}_{2}$ & $3 c$ & $\mathrm{H}$ & $4-\mathrm{ClC}_{6} \mathrm{H}_{4}$ & 100 & $38^{\mathrm{a}}(50 / 50)^{\mathrm{d}}$ \\
\hline 16 & $9 \mathbf{a}$ & $\mathrm{H}$ & $3 \mathbf{a}$ & $\mathrm{H}$ & $\mathrm{H}$ & $11 a$ & $36^{\mathrm{b}}$ \\
\hline 17 & $9 b$ & $5-\mathrm{Cl}$ & $\mathbf{3 a}$ & $\mathrm{H}$ & $\mathrm{H}$ & 11b & $38^{\mathrm{b}}$ \\
\hline 18 & $9 c$ & $5-\mathrm{Br}$ & $3 a$ & $\mathrm{H}$ & $\mathrm{H}$ & 11c & $41^{\mathrm{b}}$ \\
\hline 19 & 9d & $3-\mathrm{CH}_{3} \mathrm{O}$ & $\mathbf{3 a}$ & $\mathrm{H}$ & $\mathrm{H}$ & 11d & $42^{\mathrm{b}}$ \\
\hline 20 & $9 e$ & $5-\mathrm{NO}_{2}$ & $3 \mathbf{a}$ & $\mathrm{H}$ & $\mathrm{H}$ & $11 \mathrm{e}$ & $38^{\mathrm{b}}$ \\
\hline 21 & $9 \mathbf{a}$ & $\mathrm{H}$ & $3 b$ & $\mathrm{CH}_{3}$ & $\mathrm{CH}_{3}$ & $11 f$ & $45^{\mathrm{b}}$ \\
\hline 22 & $9 b$ & $5-\mathrm{Cl}$ & $3 b$ & $\mathrm{CH}_{3}$ & $\mathrm{CH}_{3}$ & $11 \mathrm{~g}$ & $42^{\mathrm{b}}$ \\
\hline 23 & $9 c$ & 5-Br & $3 b$ & $\mathrm{CH}_{3}$ & $\mathrm{CH}_{3}$ & $11 \mathrm{~h}$ & $40^{\mathrm{b}}$ \\
\hline 24 & 9d & $3-\mathrm{CH}_{3} \mathrm{O}$ & $3 b$ & $\mathrm{CH}_{3}$ & $\mathrm{CH}_{3}$ & $11 \mathrm{i}$ & $40^{\mathrm{b}}$ \\
\hline 25 & $9 e$ & $5-\mathrm{NO}_{2}$ & $3 b$ & $\mathrm{CH}_{3}$ & $\mathrm{CH}_{3}$ & $11 \mathrm{j}$ & $35^{\mathrm{b}}$ \\
\hline 26 & $9 \mathbf{a}$ & $\mathrm{H}$ & $3 c$ & $\mathrm{H}$ & $4-\mathrm{ClC}_{6} \mathrm{H}_{4}$ & $11 k$ & $32^{b}(50 / 50)^{d}$ \\
\hline 27 & $9 b$ & $5-\mathrm{Cl}$ & $3 c$ & $\mathrm{H}$ & $4-\mathrm{ClC}_{6} \mathrm{H}_{4}$ & 111 & $38^{\mathrm{b}}(50 / 50)^{\mathrm{d}}$ \\
\hline 28 & $9 c$ & $5-\mathrm{Br}$ & $3 c$ & $\mathrm{H}$ & $4-\mathrm{ClC}_{6} \mathrm{H}_{4}$ & $11 \mathrm{~m}$ & $32^{\mathrm{b}}(50 / 50)^{\mathrm{d}}$ \\
\hline 29 & 9d & $3-\mathrm{CH}_{3} \mathrm{O}$ & $3 c$ & $\mathrm{H}$ & $4-\mathrm{ClC}_{6} \mathrm{H}_{4}$ & $11 n$ & $34^{\mathrm{b}}(50 / 50)^{\mathrm{d}}$ \\
\hline 30 & $9 e$ & $5-\mathrm{NO}_{2}$ & $3 c$ & $\mathrm{H}$ & $4-\mathrm{ClC}_{6} \mathrm{H}_{4}$ & 110 & $38^{\mathrm{b}}(50 / 50)^{\mathrm{d}}$ \\
\hline 31 & $9 \mathbf{a}$ & $\mathrm{H}$ & $\mathbf{3 a}$ & $\mathrm{H}$ & $\mathrm{H}$ & $12 a$ & $53^{\mathrm{c}}$ \\
\hline 32 & $9 b$ & $5-\mathrm{Cl}$ & $\mathbf{3 a}$ & $\mathrm{H}$ & $\mathrm{H}$ & $12 b$ & $58^{\mathrm{c}}$ \\
\hline 33 & $9 c$ & $5-\mathrm{Br}$ & $\mathbf{3 a}$ & $\mathrm{H}$ & $\mathrm{H}$ & $12 c$ & $62^{c}$ \\
\hline 34 & 9d & $3-\mathrm{CH}_{3} \mathrm{O}$ & $3 \mathbf{a}$ & $\mathrm{H}$ & $\mathrm{H}$ & $12 d$ & $50^{\mathrm{c}}$ \\
\hline 35 & $9 e$ & $5-\mathrm{NO}_{2}$ & $3 \mathbf{a}$ & $\mathrm{H}$ & $\mathrm{H}$ & $12 \mathrm{e}$ & $55^{\mathrm{c}}$ \\
\hline
\end{tabular}


Table 3. Continued

\begin{tabular}{|c|c|c|c|c|c|c|c|}
\hline \multirow{3}{*}{$\begin{array}{l}\text { En- } \\
\text { try }\end{array}$} & \multicolumn{5}{|c|}{ Building-blocks } & \multirow{2}{*}{\multicolumn{2}{|c|}{ Reaction product }} \\
\hline & \multicolumn{2}{|c|}{ Salicylaldehyde } & & \multicolumn{2}{|c|}{$\beta$-Diketone } & & \\
\hline & & $\mathrm{R}$ & & $\mathrm{R}^{1}$ & $\mathrm{R}^{2}$ & & Yield, \% \\
\hline 36 & $9 \mathbf{a}$ & $\mathrm{H}$ & $\mathbf{3 b}$ & $\mathrm{CH}_{3}$ & $\mathrm{CH}_{3}$ & $12 f$ & $80^{\mathrm{c}}$ \\
\hline 37 & $9 b$ & $5-\mathrm{Cl}$ & $\mathbf{3 b}$ & $\mathrm{CH}_{3}$ & $\mathrm{CH}_{3}$ & $12 \mathrm{~g}$ & $75^{\mathrm{c}}$ \\
\hline 38 & $9 c$ & $5-\mathrm{Br}$ & $\mathbf{3 b}$ & $\mathrm{CH}_{3}$ & $\mathrm{CH}_{3}$ & $12 \mathrm{~h}$ & $72^{\mathrm{c}}$ \\
\hline 39 & 9d & $3-\mathrm{CH}_{3} \mathrm{O}$ & $\mathbf{3 b}$ & $\mathrm{CH}_{3}$ & $\mathrm{CH}_{3}$ & $12 \mathbf{i}$ & $68^{c}$ \\
\hline 40 & $9 e$ & $5-\mathrm{NO}_{2}$ & $\mathbf{3 b}$ & $\mathrm{CH}_{3}$ & $\mathrm{CH}_{3}$ & $12 j$ & $65^{\mathrm{c}}$ \\
\hline 41 & $9 \mathbf{a}$ & $\mathrm{H}$ & $3 c$ & $\mathrm{H}$ & $4-\mathrm{ClC}_{6} \mathrm{H}_{4}$ & $12 k$ & $69^{c}(50 / 50)^{d}$ \\
\hline 42 & $9 \mathrm{~b}$ & $5-\mathrm{Cl}$ & $3 c$ & $\mathrm{H}$ & $4-\mathrm{ClC}_{6} \mathrm{H}_{4}$ & 121 & $60^{c}(50 / 50)^{d}$ \\
\hline 43 & $9 c$ & $5-\mathrm{Br}$ & $3 c$ & $\mathrm{H}$ & $4-\mathrm{ClC}_{6} \mathrm{H}_{4}$ & $12 \mathrm{~m}$ & $62^{c}(50 / 50)^{d}$ \\
\hline 44 & 9d & $3-\mathrm{CH}_{3} \mathrm{O}$ & $3 c$ & $\mathrm{H}$ & $4-\mathrm{ClC}_{6} \mathrm{H}_{4}$ & $12 n$ & $60^{c}(50 / 50)^{d}$ \\
\hline 45 & $9 e$ & $5-\mathrm{NO}_{2}$ & $3 \mathbf{c}$ & $\mathrm{H}$ & $4-\mathrm{ClC}_{6} \mathrm{H}_{4}$ & 120 & $57^{\mathrm{c}}(50 / 50)^{\mathrm{d}}$ \\
\hline
\end{tabular}

${ }^{\mathrm{a}} \mathrm{DMF}-\mathrm{HCl}, \Delta ;{ }^{\mathrm{b}} \mathrm{DMF}, \Delta ;{ }^{\mathrm{c}}$ double excess of the aldehyde, DMF, $\Delta ;{ }^{\mathrm{d}}$ ratio (\%) of diastereomers according to ${ }^{1} \mathrm{H}$ NMR data.

NOESY spectra of compounds 4, 6, 8 and 10 contain cross-peaks of CH-group and orthoprotons of $\mathrm{R}$-substituent with $\mathrm{CH}_{3}$-group in isoxazole fragment but not with pyridine $\mathrm{NH}$ (Fig. 1) allowing excluding the formation of position isomeric structures 16. This fact was additionally proven with COSY and HMBC experiments.

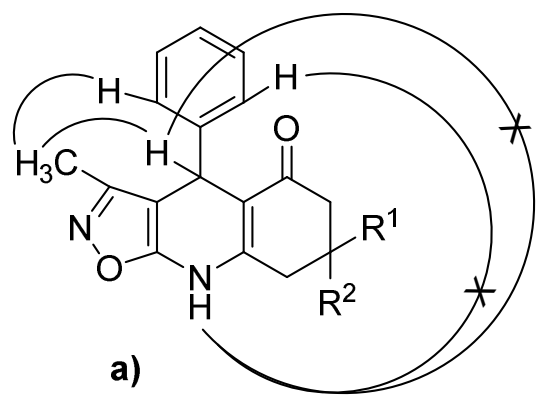<smiles>[R]C1([R])CC(=O)C2=C(C1)c1c(C)noc1NC2Br</smiles>

b)

Figure 1. Some data of NOESY experiments (a) and alternative structure (b) for compounds 4, 6 and $\mathbf{1 0 .}$

The ${ }^{1} \mathrm{H}$ NMR spectra of the compounds 11a-o and 12a-o contain no signal of NH- and CHgroup from the isoxazole moiety but exhibit broad singlets of $\mathrm{NH}_{2}$ group at 6.35-6.40 ppm (for heterocycles 11a-o) or sharp singlet of azomethine $\mathrm{CH}(8.95-9.05 \mathrm{ppm})$ and signals of protons of second aryl ring (for heterocycles 12a-o). Additionally, the structure of heterocycles 12 was proven by analysis of correlations in HSQC, HMBC and NOESY spectra. 


\section{Conclusions}

In summary, three-component heterocyclizations between 5-amino-3-methylisoxazole, derivatives of cyclohexanedione, and aromatic aldehydes were studied under conventional thermal heating, microwave irradiation, and ultrasonication. These reactions led to the formation of 4-aryl-6,7,8,9-tetrahydroisoxazolo[5,4- $b$ ]quinolin-5(4H)-ones and application of microwave activation in the most cases gave the best results from the viewpoint of yields and purity of the final compounds. Salicylic aldehyde as a substrate complicated the reaction which due to competition of $\mathrm{NH}_{2}$ and $\mathrm{OH}$ reaction centers often gave mixtures of 4-(2-hydroxyaryl)-6,7,8,9tetrahydroisoxazolo[5,4- $b$ ]quinolin-5(4H)-ones and 2,3,4,9-tetrahydro-1H-xanthen-1-ones. However, variation of the catalyst and the reaction conditions allowed tuning the selectivity of the heterocyclization. Thus, in the presence of Brønsted or Lewis acids heating of the starting materials in $\mathrm{DMF}$ or $\mathrm{H}_{2} \mathrm{O}$ led to the formation of isoxazoloquinolinones while ultrasonication in $\mathrm{EtOH}-\mathrm{Et}_{3} \mathrm{~N}$ gave only tetrahydroxanthenones.

\section{Experimental Section}

General. The melting points of all compounds synthesized were determined with a Gallenkamp melting point apparatus (for the mixture of diastereomers melting points were not measured). The NMR spectra were recorded at $400 \mathrm{MHz}\left(100 \mathrm{MHz}\right.$ for $\left.{ }^{13} \mathrm{C}\right)$ and at $200 \mathrm{MHz}(50 \mathrm{MHz}$ for ${ }^{13} \mathrm{C}$ ) with a Varian Unity Plus-400 and Varian Mercury VX-200 spectrometers, respectively. The MS spectra were measured on a GC-MS Varian 1200L (ionizing voltage $70 \mathrm{eV}$, direct input of the sample) instrument. Elemental analysis was realized on EuroVector EA-3000. Analytical samples of the compounds were obtained by their recrystallization from ethanol and further drying in vacuum at room temperature. Sonication was carried out with the help of standard ultrasonic bath producing irradiation at $44.2 \mathrm{kHz}$. Microwave experiments were performed using the Emrys Creator EXP from Biotage AB (Uppsala, Sweden) possessing a single-mode microwave cavity producing controlled irradiation at $2.45 \mathrm{GHz}$. Solvents, all reagents were commercially available and used without additional purification.

General procedure for synthesis of $4 a-z, 6 a, b$ and 8a-c. Ultrasonic-assisted synthesis. A mixture of 5-amino-3-methylisoxazole 1 (1 mmol), aldehydes $\mathbf{2 a - i}(1 \mathrm{mmol})$, and cyclic $\beta$-diketones 3a-c $(1 \mathrm{mmol})$ in ethanol $(10 \mathrm{~mL})$ was ultrasonicated at room temperature for $90 \mathrm{~min}$ in a round-bottom flask equipped with a condenser. The reaction mixture was allowed to stand up to $12 \mathrm{~h}$ at room temperature and then was filtered out to give the solid compounds, which were then washed with acetone and air dried. Reaction products were obtained in high purity and did not require further purification by recrystallization.

Thermal heating. A mixture of 5-amino-3-methylisoxazole 1 (1 mmol), aldehydes 2a-i (1 $\mathrm{mmol})$, and cyclic $\beta$-diketones 3a-c $(1 \mathrm{mmol})$ in $\mathrm{DMF}(0.1 \mathrm{~mL})$ was heated to reflux for $10 \mathrm{~min}$. 
Then after cooling acetone $(10 \mathrm{~mL})$ was added and the precipitate formed was filtered out to give the solid compounds, which were washed with acetone and air dried.

Microwave-assisted synthesis. A mixture of 5-amino-3-methylisoxazole 1 (1 mmol), aldehydes 2a-i $(1 \mathrm{mmol})$, and cyclic $\beta$-diketones 3a-c $(1 \mathrm{mmol})$ in water $(0.1 \mathrm{~mL})$ was irradiated in $\mathrm{MW}$ reactor at $120{ }^{\circ} \mathrm{C}$ for $5 \mathrm{~min}$. Then after cooling acetone $(10 \mathrm{~mL})$ was added and the precipitate formed was filtered out to give the solid compounds, which were washed with acetone and air dried.

3-Methyl-4-phenyl-4,7,8,9-tetrahydroisoxazolo[5,4-b]quinolin-5(6H)-one (4a). Colorless solid, mp 174-175 ${ }^{\circ} \mathrm{C} .{ }^{1} \mathrm{H}$ NMR (200 MHz, DMSO- $\left.d_{6}\right) \delta 10.7$ (s, 1NH), 7.29-6.97 (m, 5H), 4.96 $(\mathrm{s}, 1 \mathrm{H}), 2.57(\mathrm{~m}, 2 \mathrm{H}), 2.18(\mathrm{~m}, 2 \mathrm{H}), 1.88(\mathrm{~m}, 2 \mathrm{H}), 1.84(\mathrm{~s}, 3 \mathrm{H}) \mathrm{ppm} ;{ }^{13} \mathrm{C} \mathrm{NMR}(100 \mathrm{MHz}$, DMSO- $\left.d_{6}\right) \delta 194.42,159.02,158.31,152.26,146.66,128.02,127.93,127.44,127.08,125.94$, 111.02, 95.68, 36.87, 35.68, 27.11, 20.86, 9.78 ppm. MS (EI, $70 \mathrm{eV}): \mathrm{m} / z(\%) 280(15.1)\left[\mathrm{M}^{+}\right]$, 203 (99.9), 183 (15.5), 154 (10.7), 77 (16.3). Anal. Calcd. for $\mathrm{C}_{17} \mathrm{H}_{16} \mathrm{~N}_{2} \mathrm{O}_{2}$ : C 72.84, H 5.75, N 9.99. Found: C 72.65, H 5.63, N 9.87\%.

4-(4-Chlorophenyl)-3-methyl-4,7,8,9-tetrahydroisoxazolo[5,4-b]quinolin-5(6H)-one

(4b). Colorless solid, mp 278-279 ${ }^{\circ} \mathrm{C}$. ${ }^{1} \mathrm{H}$ NMR (200 MHz, DMSO- $\left.d_{6}\right) \delta 10.78(\mathrm{~s}, 1 \mathrm{NH}), 7.29-7.16(\mathrm{~m}$, $4 \mathrm{H}), 4.97(\mathrm{~s}, 1 \mathrm{H}), 2.56(\mathrm{~m}, 2 \mathrm{H}), 2.18(\mathrm{~m}, 2 \mathrm{H}), 1.90(\mathrm{~m}, 2 \mathrm{H}), 1.84(\mathrm{~s}, 3 \mathrm{H}) \mathrm{ppm} ;{ }^{13} \mathrm{C} \mathrm{NMR}(100$ MHz, DMSO-d $) \delta 194.94,159.61,158.80,152.95,146.08,131.01,129.85,128.47,111.23$, 107.36, 95.73, 37.34, 35.77, 27.64, 21.35, 10.27 ppm. MS (EI, $70 \mathrm{eV}): \mathrm{m} / \mathrm{z}$ (\%) 316 (9.5), 314 (20.8) $\left[\mathrm{M}^{+}\right], 313$ (36.1), 233 (31.3), 203 (78.4), 199 (99.9), 136 (52.8), 101 (29.5), 42 (87.8) Anal. Calcd. for $\mathrm{C}_{17} \mathrm{H}_{15} \mathrm{ClN}_{2} \mathrm{O}_{2}$ : C 64.87, $\mathrm{H} 4.80, \mathrm{~N}$ 8.90. Found: C 64.59, H 4.62, N 8.78\%.

4-(4-Bromophenyl)-3-methyl-4,7,8,9-tetrahydroisoxazolo[5,4-b]quinolin-5(6H)-one (4c). Colorless solid, mp 254-255 ${ }^{\circ} \mathrm{C}$. ${ }^{1} \mathrm{H}$ NMR (200 MHz, DMSO-d $) \delta 10.77(\mathrm{~s}, 1 \mathrm{NH}), 7.38-7.11(\mathrm{~m}$, $4 \mathrm{H}), 4.96(\mathrm{~s}, 1 \mathrm{H}), 2.57(\mathrm{~m}, 2 \mathrm{H}), 2.17(\mathrm{~m}, 2 \mathrm{H}), 1.88(\mathrm{~m}, 2 \mathrm{H}), 1.84(\mathrm{~s}, 3 \mathrm{H}) \mathrm{ppm} ;{ }^{13} \mathrm{C} \mathrm{NMR}(100$ MHz, DMSO-d $d_{6} \delta 194.94,159.61,158.80,152.98,146.50,131.40,130.26,119.51,111.16$, 95.66, 37.34, 35.83, 27.64, 21.35, 10.29 ppm. MS (EI, $70 \mathrm{eV}): m / z(\%) 360(7.3), 358(7.7)\left[\mathrm{M}^{+}\right]$, 279 (22.5), 203 (99.9), 154 (13.3), 127 (11.9). Anal. Calcd. for $\mathrm{C}_{17} \mathrm{H}_{15} \mathrm{BrN}_{2} \mathrm{O}_{2}$ : C 56.84, $\mathrm{H} 4.21$, N 7.80. Found: C 56.62, H 4.02, N 7.72\%.

3-Methyl-4-(4-methylphenyl)-4,7,8,9-tetrahydroisoxazolo[5,4-b]quinolin-5(6H)-one (4d). Colorless solid, mp 224-225 ${ }^{\circ} \mathrm{C} .{ }^{1} \mathrm{H}$ NMR $\left(200 \mathrm{MHz}, \mathrm{DMSO}-d_{6}\right) \delta 10.69(\mathrm{~s}, 1 \mathrm{NH}), 7.02(\mathrm{~m}, 4 \mathrm{H})$, $4.90(\mathrm{~s}, 1 \mathrm{H}), 2.55(\mathrm{~m}, 2 \mathrm{H}), 2.19(\mathrm{~s}, 3 \mathrm{H}), 2.17(\mathrm{~m}, 2 \mathrm{H}), 1.88(\mathrm{~m}, 2 \mathrm{H}), 1.84(\mathrm{~s}, 3 \mathrm{H}) \mathrm{ppm} ;{ }^{13} \mathrm{C} \mathrm{NMR}$ $\left(100 \mathrm{MHz}, \mathrm{DMSO}-d_{6}\right) \delta 194.91,158.90,152.51,144.46,135.53,129.21,128.03,96.44,37.67$, 36.02, 27.90, 21.59, 21.15, 10.35 ppm. MS (EI, $70 \mathrm{eV}): \mathrm{m} / z(\%) 294$ (22.5) [ $\left.\mathrm{M}^{+}\right], 293$ (35.5), 203 (84.0), 197 (16.1), 168 (20.1), 154 (17.7), 141 (23.1), 128 (21.6), 115 (51.5), 91 (99.9), 89 (40.1), 65 (95.2). Anal. Calcd. for $\mathrm{C}_{18} \mathrm{H}_{18} \mathrm{~N}_{2} \mathrm{O}_{2}$ : C 73.45, H 6.16, N 9.52. Found: C 73.23, H 6.08, N $9.44 \%$.

4-(4-Methoxyphenyl)-3-methyl-4,7,8,9-tetrahydroisoxazolo[5,4-b]quinolin-5(6H)-one (4e). Colorless solid, mp 219-220 ${ }^{\circ} \mathrm{C}$. ${ }^{1} \mathrm{H}$ NMR (200 MHz, DMSO- $\left.d_{6}\right) \delta 10.67$ (s, $\left.1 \mathrm{NH}\right), 7.08-7.64(\mathrm{~m}$, $4 \mathrm{H}), 4.90(\mathrm{~s}, 1 \mathrm{H}), 3.66(\mathrm{~s}, 3 \mathrm{H}), 2.52(\mathrm{~m}, 2 \mathrm{H}), 2.17(\mathrm{~m}, 2 \mathrm{H}), 1.88(\mathrm{~m}, 2 \mathrm{H}), 1.84(\mathrm{~s}, 3 \mathrm{H}) \mathrm{ppm} ;{ }^{13} \mathrm{C}$ NMR $\left(100 \mathrm{MHz}, \mathrm{DMSO}-d_{6}\right) \delta 194.90,167.92,159.88,152.29,139.73,129.78,114.32,112.37$, 
96.52, 55.76, 37.71, 35.60, 34.55, 21.63, 10.29 ppm. MS (EI, $70 \mathrm{eV}): \mathrm{m} / \mathrm{z}(\%) 310(47.0)\left[\mathrm{M}^{+}\right]$, 309 (74.8), 295 (60.4), 269 (17.2), 213 (22.9), 203 (99.9), 184 (14.9), 77 (13.1), 62 (11.8). Anal. Calcd. for $\mathrm{C}_{18} \mathrm{H}_{18} \mathrm{~N}_{2} \mathrm{O}_{3}$ : C 69.66, H 5.85, N 9.03. Found: C 69.58, H 5.63, N 8.95\%.

\section{3-Methyl-4-(4-nitrophenyl)-4,7,8,9-tetrahydroisoxazolo[5,4-b]quinolin-5(6H)-one}

(4f). Colorless solid, mp 217-218 ${ }^{\circ} \mathrm{C}$. ${ }^{1} \mathrm{H}$ NMR (200 MHz, DMSO- $\left.d_{6}\right) \delta 10.90(\mathrm{~s}, 1 \mathrm{NH}), 8.10-7.47$ (m, $4 \mathrm{H}), 5.14(\mathrm{~s}, 1 \mathrm{H}), 2.60(\mathrm{~m}, 2 \mathrm{H}), 2.17(\mathrm{~m}, 2 \mathrm{H}), 1.88(\mathrm{~m}, 2 \mathrm{H}), 1.84(\mathrm{~s}, 3 \mathrm{H}) \mathrm{ppm} ;{ }^{13} \mathrm{C} \mathrm{NMR}(100$ MHz, DMSO- $\left.d_{6}\right) \delta 194.96,159.75,158.81,154.42,153.58,146.33,130.28,123.91,110.62$, 107.36, 95.12, 37.25, 36.54, 27.66, 21.33, 10.30 ppm. MS (EI, $70 \mathrm{eV}): \mathrm{m} / z(\%) 325(9.4)\left[\mathrm{M}^{+}\right]$, 324 (14.3), 228 (26.4), 203 (99.9), 77 (12.5), 42 (16.9). Anal. Calcd. for $\mathrm{C}_{17} \mathrm{H}_{15} \mathrm{~N}_{3} \mathrm{O}_{4}$ : C 62.76, $\mathrm{H}$ 4.65, N 12.92. Found: C 62.54, H 4.47, N $12.80 \%$

Methyl 4-(3-methyl-5-oxo-4,5,6,7,8,9-hexahydroisoxazolo[5,4-b]quinolin-4-yl)benzoate (4g). Colorless solid, mp 292-203 ${ }^{\circ} \mathrm{C} .{ }^{1} \mathrm{H}$ NMR (200 MHz, DMSO- $\left.d_{6}\right) \delta 10.81(\mathrm{~s}, 1 \mathrm{NH}), 7.75-7.30(\mathrm{~m}$, $4 \mathrm{H}), 5.05(\mathrm{~s}, 1 \mathrm{H}), 3.79(\mathrm{~s}, 3 \mathrm{H}), 2.60(\mathrm{~m}, 2 \mathrm{H}), 2.17(\mathrm{~m}, 2 \mathrm{H}), 1.88(\mathrm{~m}, 2 \mathrm{H}), 1.84(\mathrm{~s}, 3 \mathrm{H}) \mathrm{ppm} ;{ }^{13} \mathrm{C}$ NMR (100 MHz, DMSO-d $) \delta 194.94,166.60,159.65,158.25,153.24,152.35,129.62,129.24$, 128.40, 110.96, 95.53, 52.47, 36.51, 33.30, 27.66, 21.35, $10.15 \mathrm{ppm}$. MS (EI, $70 \mathrm{eV}): \mathrm{m} / \mathrm{z}(\%)$ 338 (4.7) $\left[\mathrm{M}^{+}\right], 258$ (12.7), 257 (17.4), 243 (99.9), 199 (76.3), 157 (23.8), 129 (46.4), 115 (31.9), 101 (56.2), 84 (72.0), 42 (83.8). Anal. Calcd. for $\mathrm{C}_{19} \mathrm{H}_{18} \mathrm{~N}_{2} \mathrm{O}_{4}$ : C 67.44, H 5.36, N 8.28. Found: C $67.27, \mathrm{H} 5.23, \mathrm{~N} 8.16 \%$.

\section{4-[4-(Dimethylamino)phenyl]-3-methyl-4,7,8,9-tetrahydroisoxazolo[5,4-b]quinolin-5(6H)-}

one (4h). Colorless solid, mp 240-241 ${ }^{\circ} \mathrm{C} .{ }^{1} \mathrm{H}$ NMR $\left(200 \mathrm{MHz}, \mathrm{DMSO}-d_{6}\right) \delta 10.60$ (s, 1NH), 6.94-6.55 (m, 4H), $4.82(\mathrm{~s}, 1 \mathrm{H}), 2.79(\mathrm{~s}, 6 \mathrm{H}), 2.54(\mathrm{~m}, 2 \mathrm{H}), 2.17(\mathrm{~m}, 2 \mathrm{H}), 1.88(\mathrm{~m}, 2 \mathrm{H}), 1.85(\mathrm{~s}$, $3 \mathrm{H}) \mathrm{ppm} ;{ }^{13} \mathrm{C}$ NMR $(100 \mathrm{MHz}$, DMSO-d $) \delta 194.42,158.92,158.35,151.99,148.77,135.84$, $127.93,112.31,111.63,96.06,40.32,36.95,34.34,27.10,20.89,9.76$ ppm. MS (EI, $70 \mathrm{eV}): \mathrm{m} / z$ (\%) 323 (34.4) $\left[\mathrm{M}^{+}\right], 308$ (23.6), 282 (99.9), 283 (18.7), 281 (59.4), 205 (17.0), 120 (20.2), 77 (10.7). Anal. Calcd. for $\mathrm{C}_{19} \mathrm{H}_{21} \mathrm{~N}_{3} \mathrm{O}_{2}$ : C 70.57, $\mathrm{H}$ 6.55, N 12.99. Found: C 70.39, $\mathrm{H}$ 6.37, N $12.87 \%$.

4-Isopropyl-3-methyl-4,7,8,9-tetrahydroisoxazolo[5,4- $b$ ]quinolin-5(6H)-one (4i). Colorless solid, mp 190-191 ${ }^{\circ} \mathrm{C} .{ }^{1} \mathrm{H}$ NMR (200 MHz, DMSO- $\left.d_{6}\right) \delta 10.60(\mathrm{~s}, 1 \mathrm{NH}), 3.84(\mathrm{~d}, J 3.1 \mathrm{~Hz}, 1 \mathrm{H})$, $2.48(\mathrm{~m}, 2 \mathrm{H}), 2.26(\mathrm{~m}, 2 \mathrm{H}), 2.11(\mathrm{~s}, 3 \mathrm{H}), 1.88(\mathrm{~m}, 2 \mathrm{H}), 1.66(\mathrm{~m}, 1 \mathrm{H}), 0.76(\mathrm{~d}, J 7.0 \mathrm{~Hz}, 3 \mathrm{H}), 0.57$ $(\mathrm{d}, J 7.0 \mathrm{~Hz}, 3 \mathrm{H}) \mathrm{ppm} ;{ }^{13} \mathrm{C}$ NMR $\left(100 \mathrm{MHz}, \mathrm{DMSO}-d_{6}\right) \delta 194.40,160.92,156.35,148.87$, 106.63, 94.06, 38.32, 36.85, 29.18, 27.80, 23.10, 22.20, 20.80, $12.76 \mathrm{ppm}$. MS (EI, $70 \mathrm{eV}): \mathrm{m} / \mathrm{z}$ (\%) 246 (31.3) $\left[\mathrm{M}^{+}\right], 203$ (56.7), 172 (99.9), 97 (44.5), 77 (14.1). Anal. Calcd. for $\mathrm{C}_{14} \mathrm{H}_{18} \mathrm{~N}_{2} \mathrm{O}_{2}$ : C 68.27, H 7.37, N 11.37. Found: C 68.15, H 7.28, N 11.19\%.

\section{3,7,7-Trimethyl-4-phenyl-4,7,8,9-tetrahydroisoxazolo $[5,4-b]$ quinolin-5(6H)-one}

(4j). Colorless solid, mp 183-184 ${ }^{\circ} \mathrm{C}$. ${ }^{1} \mathrm{H}$ NMR (200 MHz, DMSO- $\left.d_{6}\right) \delta 10.68(\mathrm{~s}, 1 \mathrm{NH}), 7.26-7.16(\mathrm{~m}$, $5 \mathrm{H}), 4.96(\mathrm{~s}, 1 \mathrm{H}), 2.51(\mathrm{~d}, J 17.1 \mathrm{~Hz}, 1 \mathrm{H}), 2.38(\mathrm{~d}, J 17.1 \mathrm{~Hz}, 1 \mathrm{H}), 2.16(\mathrm{~d}, J 16.1 \mathrm{~Hz}, 1 \mathrm{H}), 1.99$ $(\mathrm{d}, J 16.1 \mathrm{~Hz}, 1 \mathrm{H}), 1.83$ (s, 3H), $1.00(\mathrm{~s}, 3 \mathrm{H}), 0.93$ (s, 3H) ppm; ${ }^{13} \mathrm{C}$ NMR (100 MHz, DMSO- $\left.d_{6}\right)$ $\delta 194.62,159.63,158.84,150.78,147.12,128.52,127.93,126.49,110.35,96.29,50.89,40.89$,

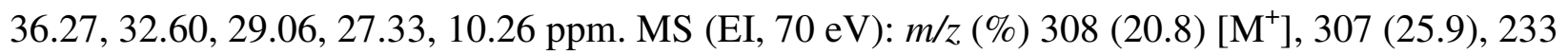


(19.6), 231 (99.9), 183 (16.0), 175 (11.1), 128 (13.1), 77 (7.1). Anal. Calcd. for $\mathrm{C}_{19} \mathrm{H}_{20} \mathrm{~N}_{2} \mathrm{O}_{2}$ : C 74.00, H 6.54, N 9.08. Found: C 73.82, H 6.25, N 9.00\%.

\section{4-(4-Chlorophenyl)-3,7,7-trimethyl-4,7,8,9-tetrahydroisoxazolo[5,4-b]quinolin-5(6H)-one}

(4k). Colorless solid, mp 270-271 ${ }^{\circ} \mathrm{C}$. ${ }^{1} \mathrm{H}$ NMR (200 MHz, DMSO- $\left.d_{6}\right) \delta 10.74$ (s, 1NH), 7.23$7.10(\mathrm{~m}, 4 \mathrm{H}), 4.93(\mathrm{~s}, 1 \mathrm{H}), 2.51(\mathrm{~d}, J 17.1 \mathrm{~Hz}, 1 \mathrm{H}), 2.38(\mathrm{~d}, J 17.1 \mathrm{~Hz}, 1 \mathrm{H}), 2.15(\mathrm{~d}, J 16.1 \mathrm{~Hz}$, $1 \mathrm{H}), 1.99$ (d, J 16.1 Hz, 1H), 1.84 (s, 3H), 1.00 (s, 3H), 0.93 (s, 3H) ppm; ${ }^{13} \mathrm{C} \mathrm{NMR}(100 \mathrm{MHz}$, DMSO- $\left.d_{6}\right) \delta 194.65,159.67,158.94,151.78,148.12,129.52,128.92,128.63,111.35,97.29$, 51.89, 41.09, 36.87, 32.80, 29.66, 28.33, 11.26 ppm. MS (EI, $70 \mathrm{eV}): \mathrm{m} / z$ (\%) 344 (1.5), 342 (4.4) $\left[\mathrm{M}^{+}\right], 248$ (35.8), 231 (99.9), 171 (14.8), 111 (17.4), 77 (12.8). Anal. Calcd. for $\mathrm{C}_{19} \mathrm{H}_{19} \mathrm{ClN}_{2} \mathrm{O}_{2}$ : C 66.57, H 5.59, N 8.17. Found: C 66.35, H 5.40, N 8.05\%.

\section{4-(4-Bromophenyl)-3,7,7-trimethyl-4,7,8,9-tetrahydroisoxazolo[5,4-b]quinolin-5(6H)-one}

(4l). Colorless solid, mp 224-225 ${ }^{\circ} \mathrm{C}$. ${ }^{1} \mathrm{H}$ NMR (200 MHz, DMSO- $\left.d_{6}\right) \delta 10.74(\mathrm{~s}, 1 \mathrm{NH}), 7.42-$ $7.10(\mathrm{~m}, 4 \mathrm{H}), 4.94(\mathrm{~s}, 1 \mathrm{H}), 2.51(\mathrm{~d}, J 17.2 \mathrm{~Hz}, 1 \mathrm{H}), 2.38(\mathrm{~d}, J 17.2 \mathrm{~Hz}, 1 \mathrm{H}), 2.15$ (d, J $16.2 \mathrm{~Hz}$, 1H), 1.99 (d, J 16.1 Hz, 1H), 1.84 (s, 3H), 1.00 (s, 3H), 0.93 (s, 3H) ppm; ${ }^{13} \mathrm{C}$ NMR (100 MHz, DMSO-d $d_{6} \delta 194.63,159.70,158.82,150.97,146.44,131.40,130.24,119.52,109.97,95.75$, 50.82, 40.86, 35.89, 32.59, 29.00, 27.35, 10.26 ppm. MS (EI, $70 \mathrm{eV}): \mathrm{m} / z$ (\%) 388 (5.3), 386 (5.4) $\left[\mathrm{M}^{+}\right], 307$ (17.6), 231 (99.9), 154 (11.3), 62 (12.3). Anal. Calcd. for $\mathrm{C}_{19} \mathrm{H}_{19} \mathrm{BrN}_{2} \mathrm{O}_{2}$ : C 58.93, H 4.95, N 7.23. Found: C 58.75, H 4.76, N 7.11\%.

\section{3,7,7-Trimethyl-(4-methylphenyl)-4,7,8,9-tetrahydroisoxazolo[5,4-b]quinolin-5(6H)-one}

(4m). Colorless solid, mp 229-230 ${ }^{\circ} \mathrm{C} .{ }^{1} \mathrm{H}$ NMR (200 MHz, DMSO- $\left.d_{6}\right) \delta 10.60(\mathrm{~s}, 1 \mathrm{NH}), 7.02$ (m, 4H), 4.89 (s, 1H), 2.51 (d, J 17.1 Hz, 1H), 2.38 (d, J 17.1 Hz, 1H), 2.19 (s, 3H), 2.15 (d, $J$ $16.1 \mathrm{~Hz}, 1 \mathrm{H}), 1.99(\mathrm{~d}, J 16.1 \mathrm{~Hz}, 1 \mathrm{H}), 1.84(\mathrm{~s}, 3 \mathrm{H}), 1.00(\mathrm{~s}, 3 \mathrm{H}), 0.93(\mathrm{~s}, 3 \mathrm{H}) \mathrm{ppm} ;{ }^{13} \mathrm{C}$ NMR $\left(100 \mathrm{MHz}, \mathrm{DMSO}-d_{6}\right) \delta 194.83,160.70,152.82$, 147.97, 139.44, 137.40, 129.24, 128.52, 106.97, 96.75, 51.82, 40.96, 36.89, 33.59, 29.80, 27.45, 21.82, $10.26 \mathrm{ppm}$. MS (EI, $70 \mathrm{eV}): \mathrm{m} / \mathrm{z}$ (\%) 322 (53.48) $\left[\mathrm{M}^{+}\right], 265$ (28.8), 231 (99.9), 227 (46.5), 179 (56), 97 (14.3). Anal. Calcd. for $\mathrm{C}_{20} \mathrm{H}_{22} \mathrm{~N}_{2} \mathrm{O}_{2}$ : C 74.51, H 6.88, N 8.69. Found: C 74.30, H 6.57, N 8.61\%.

\section{4-(4-Methoxylphenyl)-3,7,7-trimethyl-4,7,8,9-tetrahydroisoxazolo[5,4-b]quinolin-5(6H)-one} (4n). Colorless solid, mp 226-227 ${ }^{\circ} \mathrm{C}$. ${ }^{1} \mathrm{H}$ NMR (200 MHz, DMSO- $\left.d_{6}\right) \delta 10.66(\mathrm{~s}, 1 \mathrm{NH}), 7.05-$ $6.75(\mathrm{~m}, 4 \mathrm{H}), 4.90(\mathrm{~s}, 1 \mathrm{H}), 3.66(\mathrm{~s}, 3 \mathrm{H}), 2.51(\mathrm{~d}, J 17.2 \mathrm{~Hz}, 1 \mathrm{H}), 2.38(\mathrm{~d}, J 17.2 \mathrm{~Hz}, 1 \mathrm{H}), 2.15$ (d, $J 16.2 \mathrm{~Hz}, 1 \mathrm{H}), 1.99(\mathrm{~d}, J 16.2 \mathrm{~Hz}, 1 \mathrm{H}), 1.84(\mathrm{~s}, 3 \mathrm{H}), 1.00(\mathrm{~s}, 3 \mathrm{H}), 0.93(\mathrm{~s}, 3 \mathrm{H}) \mathrm{ppm} ;{ }^{13} \mathrm{C} \mathrm{NMR}$ $\left(100 \mathrm{MHz}, \mathrm{DMSO}-d_{6}\right) \delta 195.02,159.84,158.93,152.32,150.39,139.61,129.11,113.97,112.30$, 96.53, 55.72, 52.28, 37.69, 35.66, 32.66, 27.90, 21.58, 10.33 ppm. MS (EI, $70 \mathrm{eV}): \mathrm{m} / \mathrm{z}(\%) 338$ (52.2) $\left[\mathrm{M}^{+}\right], 337$ (47.7), 323 (80.9), 309 (22.6), 233 (99.9), 213 (19.9), 184 (21.9), 170 (18.4), 127 (29.2), 108 (62.9), 92 (62.1), 77 (85.8), 41 (40.9). Anal. Calcd. for $\mathrm{C}_{20} \mathrm{H}_{22} \mathrm{~N}_{2} \mathrm{O}_{3}$ : C 70.99, $\mathrm{H}$ 6.55 , N 8.28. Found: C 70.77, H 6.27, N 8.20\%.

\section{3,7,7-Trimethyl-4-(4-nitrophenyl)-4,7,8,9-tetrahydroisoxazolo[5,4-b]quinolin-5(6H)-one} (4o). Colorless solid, mp 232-233 ${ }^{\circ} \mathrm{C} .{ }^{1} \mathrm{H}$ NMR (200 MHz, DMSO- $\left.d_{6}\right) \delta 10.89$ (s, 1NH), 8.13$7.44(\mathrm{~m}, 4 \mathrm{H}), 5.13(\mathrm{~s}, 1 \mathrm{H}), 2.57$ (d, J 17.1 Hz, 1H), 2.38 (d, J 17.1 Hz, 1H), 2.18 (d, J 16.1 Hz, 1H), 1.99 (d, J 16.1 Hz, 1H), 1.84 (s, 3H), 1.00 (s, 3H), 0.93 (s, 3H) ppm; ${ }^{13} \mathrm{C} \mathrm{NMR} \mathrm{(100} \mathrm{MHz,}$ DMSO- $\left.d_{6}\right) \delta 194.70,170.85,154.37,152.01,150.44,149.09,129.68,129.02,95.35,50.80$, 
45.71, 36.94, 32.80, 29.30, 27.75, 11.42 ppm. MS (EI, $70 \mathrm{eV}): \mathrm{m} / \mathrm{z}(\%) 353(12.8)\left[\mathrm{M}^{+}\right], 233$ (50.4), 231 (99.9), 228 (30.8), 175 (17.4), 127 (10.4), 100 (12.1), 98 (14.2), 64 (18.8), 41 (28.2). Anal. Calcd. for $\mathrm{C}_{19} \mathrm{H}_{19} \mathrm{~N}_{3} \mathrm{O}_{4}$ : C 64.58, H 5.42, N 11.89. Found: C 64.37, H 5.10, N 11.80\%.

\section{Methyl-4-(3,7,7-trimethyl-5-oxo-4,5,6,7,8,9-hexahydroisoxazolo[5,4-b]quinolin-4-}

yl)benzoate (4p). Colorless solid, mp 242-243 ${ }^{\circ} \mathrm{C} .{ }^{1} \mathrm{H}$ NMR $\left(200 \mathrm{MHz}, \mathrm{DMSO}-d_{6}\right) \delta 10.78(\mathrm{~s}$, $1 \mathrm{NH}), 7.80-7.30(\mathrm{~m}, 4 \mathrm{H}), 5.03(\mathrm{~s}, 1 \mathrm{H}), 3.79$ (s, 3H), 2.53 (d, J 17.0 Hz, 1H), 2.40 (d, J $17.0 \mathrm{~Hz}$, 1H), $2.19(\mathrm{~d}, J 16.0 \mathrm{~Hz}, 1 \mathrm{H}), 1.97(\mathrm{~d}, J 16.0 \mathrm{~Hz}, 1 \mathrm{H}), 1.82(\mathrm{~s}, 3 \mathrm{H}), 1.00(\mathrm{~s}, 3 \mathrm{H}), 0.92(\mathrm{~s}, 3 \mathrm{H})$ ppm; ${ }^{13} \mathrm{C}$ NMR (100 MHz, DMSO- $\left.d_{6}\right) \delta 194.12,166.07,159.25,158.33,151.77,150.69,129.10$, $127.88,127.70,127.47,109.28,95.10,51.94,50.26,35.99,32.10,30.65,28.48,26.77,9.72$ ppm. MS (EI, $70 \mathrm{eV}): \mathrm{m} / z$ (\%) 366 (15.7) $\left[\mathrm{M}^{+}\right], 231$ (99.9), 215 (5.6), 175 (5.2), 154 (5.1), 104 (4.9), 77 (4.2). Anal. Calcd. for $\mathrm{C}_{21} \mathrm{H}_{22} \mathrm{~N}_{2} \mathrm{O}_{4}$ : C 68.84, H 6.05, N 7.65. Found: C 68.61, H 5.73, N $7.53 \%$.

4-[4-(Dimethylamino)phenyl]-3,7,7-trimethyl-4,7,8,9-tetrahydroisoxazolo[5,4-b]quinolin5(6H)-one (4q). Colorless solid, mp 251-252 ${ }^{\circ} \mathrm{C} .{ }^{1} \mathrm{H}$ NMR (200 MHz, DMSO- $\left.d_{6}\right) \delta 10.54$ (s, $1 \mathrm{NH}), 6.93-6.54(\mathrm{~m}, 4 \mathrm{H}), 4.80(\mathrm{~s}, 1 \mathrm{H}), 2.79$ (s, 3H), 2.48 (d, J 16.3 Hz, 1H), 2.31 (d, J $16.3 \mathrm{~Hz}$, 1H), 2.15 (d, J 16.3 Hz, 1H), 1.99 (d, J 16.3 Hz, 1H), $1.84(\mathrm{~s}, 3 \mathrm{H}), 1.00(\mathrm{~s}, 3 \mathrm{H}), 0.93$ (s, 3H) ppm; ${ }^{13} \mathrm{C}$ NMR (100 MHz, DMSO- $\left.d_{6}\right) \delta 194.62,159.47,158.88,150.03,149.25,135.28,128.41$, $112.61,110.89,107.36,96.71,50.99,40.72,39.55,35.03,32.56,29.16,27.30,10.26$ ppm. MS

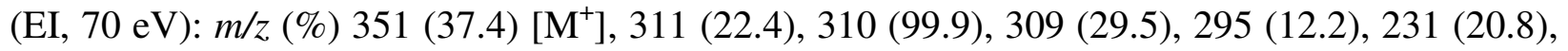
77 (14.5), 62 (11.8). Anal. Calcd. for $\mathrm{C}_{21} \mathrm{H}_{25} \mathrm{~N}_{3} \mathrm{O}_{2}$ : C 71.77, $\mathrm{H}$ 7.17, N 11.96. Found: C 71.53, $\mathrm{H}$ $6.82, \mathrm{~N} 11.80 \%$.

4-Isopropyl-3,7,7-trimethyl-4,7,8,9-tetrahydroisoxazolo[5,4-b]quinolin-5(6H)-one (4r). Colorless solid, mp 225-226 ${ }^{\circ} \mathrm{C} .{ }^{1} \mathrm{H}$ NMR $\left(200 \mathrm{MHz}, \mathrm{DMSO}-d_{6}\right) \delta 10.41$ (s, 1NH), $3.80(\mathrm{~d}, J 3.0$ $\mathrm{Hz}, 1 \mathrm{H}), 2.44$ (d, J 17.0 Hz, 1H), 2.27 (d, J 17.0 Hz, 1H), 2.22 (d, J 16.5 Hz, 1H), 2.05 (d, J 16.5 $\mathrm{Hz}, 1 \mathrm{H}), 2.11(\mathrm{~s}, 3 \mathrm{H}), 1.69(\mathrm{~m}, 1 \mathrm{H}), 1.00(\mathrm{~s}, 3 \mathrm{H}), 0.98(\mathrm{~s}, 3 \mathrm{H}), 0.78$ (d, J 7.0 Hz, 3H), 0.56 (d, J $7.0 \mathrm{~Hz}, 3 \mathrm{H}) \mathrm{ppm} ;{ }^{13} \mathrm{C}$ NMR (100 MHz, DMSO- $\left.d_{6}\right) \delta 195.01,162.00,158.90,152.19,109.65$, 107.36, 92.67, 51.03, 40.80, 35.57, 34.42, 32.38, 29.48, 27.07, 20.70, 17.66, 11.18 ppm. MS (EI, $70 \mathrm{eV}$ ): $\mathrm{m} / \mathrm{z}(\%) 274$ (11.9) $\left[\mathrm{M}^{+}\right], 170$ (99.9), 123 (36.6), 97 (23.3). Anal. Calcd. for $\mathrm{C}_{16} \mathrm{H}_{22} \mathrm{~N}_{2} \mathrm{O}_{2}$ : C 70.04, H 8.08, N 10.21. Found: C 70.01, H 7.82, N 10.13\%.

7-(4-Chlorophenyl)-3-methyl-4-phenyl-4,7,8,9-tetrahydroisoxazolo[5,4- $b$ ]quinolin-5(6H)one (4s). Colorless solid; ${ }^{1} \mathrm{H}$ NMR (200 MHz, DMSO- $\left.d_{6}\right) \delta 10.84$ (10.31) (s, 1NH), 7.46-7.01 $(\mathrm{m}, 10 \mathrm{H}), 5.84$ (5.65) (s, 1H), $3.42(\mathrm{~m}, 1 \mathrm{H}), 3.01-2.70(\mathrm{~m}, 2 \mathrm{H}), 2.53-2.34(\mathrm{~m}, 2 \mathrm{H}), 2.16(1.85)(\mathrm{s}$, $3 \mathrm{H}) \mathrm{ppm} ;{ }^{13} \mathrm{C}$ NMR (100 MHz, DMSO- $\left.d_{6}\right) \delta 195.05,160.80,151.86,145.08,141.20,131.09$, $130.39,127.17,127.01,126.08,124.90,106.58,95.05,40.85,36.38,36.09,31.07,11.05$ ppm.

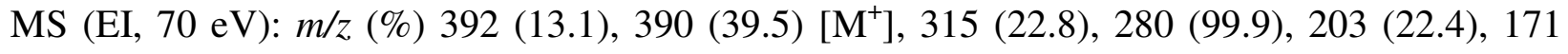
(18.8), 77 (45.4). Anal. Calcd. for $\mathrm{C}_{23} \mathrm{H}_{19} \mathrm{ClN}_{2} \mathrm{O}_{2}: \mathrm{C} 70.68, \mathrm{H} 4.90, \mathrm{~N} 7.17$. Found: C 70.43, H $4.55, \mathrm{~N} 7.02 \%$.

4,7-Bis-(4-chlorophenyl)-3-methyl-4,7,8,9-tetrahydroisoxazolo[5,4-b]quinolin-5(6H)-one (4t). Colorless solid; ${ }^{1} \mathrm{H}$ NMR (200 MHz, DMSO- $\left.d_{6}\right) \delta 10.99(10.89)(\mathrm{s}, 1 \mathrm{NH}), 7.47-6.98$ (m, $8 \mathrm{H}), 4.99$ (4.98) (s, 1H), $3.42(\mathrm{~m}, 1 \mathrm{H}), 3.01-2.70(\mathrm{~m}, 2 \mathrm{H}), 2.53-2.34(\mathrm{~m}, 2 \mathrm{H}), 2.41(2.39)(\mathrm{s}, 3 \mathrm{H})$ 
ppm; ${ }^{13} \mathrm{C}$ NMR (100 MHz, DMSO- $\left.d_{6}\right) \delta 194.50,158.9,151.76,146.10,139.04,131.59,128.70$, 128.07, 125.68, 108.56, 95.23, 44.59, 36.78, 36.09, 30.72, $10.45 \mathrm{ppm}$. MS (EI, $70 \mathrm{eV}): \mathrm{m} / \mathrm{z}(\%)$ 426 (8.5), 424 (12.8) $\left[\mathrm{M}^{+}\right], 314$ (99.9.), 202 (56.5), 96 (11.2), 77 (48.6). Anal. Calcd. for $\mathrm{C}_{23} \mathrm{H}_{18} \mathrm{Cl}_{2} \mathrm{~N}_{2} \mathrm{O}_{2}$ : C 64.95, H 4.27, N 6.59. Found: C 64.71, H 4.10, N 6.44\%.

4-(4-Bromophenyl)-7-(4-chlorophenyl)-3-methyl-4,7,8,9-tetrahydroisoxazolo[5,4b] quinolin-5(6H)-one (4u). Colorless solid, mp 248-249 ${ }^{\circ} \mathrm{C} .{ }^{1} \mathrm{H}$ NMR (200 MHz, DMSO- $\left.d_{6}\right) \delta$ $10.88(\mathrm{~s}, 1 \mathrm{NH}), 7.39-7.05(\mathrm{~m}, 8 \mathrm{H}), 4.97(\mathrm{~s}, 1 \mathrm{H}), 3.42(\mathrm{~m}, 1 \mathrm{H}), 3.01-2.70(\mathrm{~m}, 2 \mathrm{H}), 2.53-2.34(\mathrm{~m}$, $2 \mathrm{H}), 1.85(\mathrm{~s}, 3 \mathrm{H}) \mathrm{ppm} ;{ }^{13} \mathrm{C}$ NMR $\left(100 \mathrm{MHz}, \mathrm{DMSO}-d_{6}\right) \delta 193.83,159.53,158.83,151.76$, 146.14, 142.57, 131.71, 131.26, 130.31, 129.35, 128.86, 119.50, 110.81, 107.36, 95.67, 44.08, 36.07, 34.31, 32.28, 10.34 ppm. MS (EI, $70 \mathrm{eV}): \mathrm{m} / \mathrm{z}$ (\%) 472 (14.4), 470 (57.8), 468 (43.2) $\left[\mathrm{M}^{+}\right], \quad 370$ (18.5), 202 (12.2), 172 (99.9), 154 (18.9), 77 (28.9). Anal. Calcd. for $\mathrm{C}_{23} \mathrm{H}_{18} \mathrm{BrClN}_{2} \mathrm{O}_{2}$ : C 58.81, H 3.86, N 5.96. Found: C 58.58, H 3.54, N 5.82\%.

7-(4-Chlorophenyl)-3-methyl-4-(4-methylphenyl)-4,7,8,9-tetrahydroisoxazolo[5,4-b]quinolin-5(6H)-one (4v). Colorless solid; ${ }^{1} \mathrm{H}$ NMR (200 MHz, DMSO- $\left.d_{6}\right) \delta 10.78(\mathrm{~s}, 1 \mathrm{NH}), 7.37-6.97$ $(\mathrm{m}, 8 \mathrm{H}), 4.92(\mathrm{~s}, 1 \mathrm{H}), 3.46(\mathrm{~m}, 1 \mathrm{H}), 3.01-2.86(\mathrm{~m}, 2 \mathrm{H}), 2.78-2.58(\mathrm{~m}, 2 \mathrm{H}), 2.20(\mathrm{~s}, 3 \mathrm{H}), 1.85(\mathrm{~s}$, $3 \mathrm{H}) \mathrm{ppm} ;{ }^{13} \mathrm{C}$ NMR $\left(100 \mathrm{MHz}\right.$, DMSO- $\left.d_{6}\right) \delta 193.79,159.41,158.85,151.29,143.91,142.67$, 135.37, 131.67, 129.38, 127.94, 111.35, 96.25, 44.16, 38.38, 36.01, 34.38, 21.08, 10.34 ppm. MS

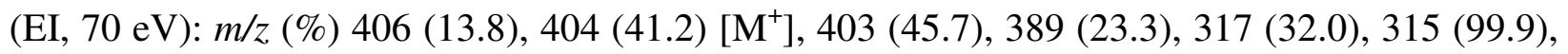
313 (94.6), 264 (19.0), 197 (32.3), 125 (26.9), 77 (11.5). Anal. Calcd. for $\mathrm{C}_{24} \mathrm{H}_{21} \mathrm{ClN}_{2} \mathrm{O}_{2}$ : C 71.19, H 5.23, N 6.92. Found: C 71.02, H 5.02, N 6.79\%.

7-(4-Chlorophenyl)-4-(4-methoxyphenyl)-3-methyl-4,7,8,9-tetrahydroisoxazolo[5,4-b]quinolin-5(6H)-one (4w). Colorless solid; ${ }^{1} \mathrm{H}$ NMR (200 MHz, DMSO- $\left.d_{6}\right) \delta 10.78$ (s, 1NH), 7.34$6.72(\mathrm{~m}, 8 \mathrm{H}), 4.91(\mathrm{~s}, 1 \mathrm{H}), 3.76(\mathrm{~s}, 3 \mathrm{H}), 3.41(\mathrm{~m}, 1 \mathrm{H}), 3.01-2.86(\mathrm{~m}, 2 \mathrm{H}), 2.78-2.58(\mathrm{~m}, 2 \mathrm{H})$, 1.85 (s, 3H) ppm; ${ }^{13} \mathrm{C}$ NMR $\left(100 \mathrm{MHz}\right.$, DMSO- $\left.d_{6}\right) \delta 193.82,159.39,158.86,157.91,151.11$, 142.66, 139.10, 131.65, 129.38, 128.84, 113.73, 111.49, 96.34, 55.42, 44.18, 38.35, 35.49, 34.34,

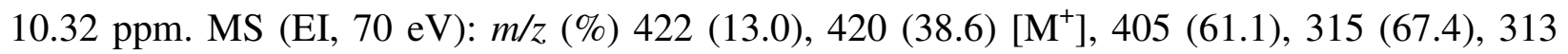
(99.9), 175 (13.0), 135 (15.3), 101 (15.2) Anal. Calcd. for $\mathrm{C}_{24} \mathrm{H}_{21} \mathrm{ClN}_{2} \mathrm{O}_{3}: \mathrm{C}$ 68.49, H 5.03, N 6.66. Found: C 68.32, H 4.70, N 6.51\%.

7-(4-Chlorophenyl)-3-methyl-4-(4-nitrophenyl)-4,7,8,9-tetrahydroisoxazolo[5,4-b]quinolin5(6H)-one (4x). Colorless solid; ${ }^{1} \mathrm{H}$ NMR (200 MHz, DMSO- $\left.d_{6}\right) \delta 11.15(10.39)(\mathrm{s}, 1 \mathrm{NH}), 8.05-$ $7.30(\mathrm{~m}, 8 \mathrm{H}), 5.66(5.15)(\mathrm{s}, 1 \mathrm{H}), 3.45(\mathrm{~m}, 1 \mathrm{H}), 3.01-2.79(\mathrm{~m}, 2 \mathrm{H}), 2.78-2.31(\mathrm{~m}, 2 \mathrm{H}), 2.15(1.85)$ (s, 3H) ppm; ${ }^{13} \mathrm{C}$ NMR (100 MHz, DMSO- $\left.d_{6}\right) \delta 195.40,159.80,153.86,148.80,145.50,133.10$, $130.90,127.10,126.48,123.88,110.05,96.53,40.89,38.88,37.80,31.20,10.50$ ppm. MS (EI, $70 \mathrm{eV}): \mathrm{m} / z$ (\%) 437 (1.9), 435 (5.3) [ $\left.\mathrm{M}^{+}\right], 324$ (99.9), 203 (18.9), 97 (22.5), 77 (58.9). Anal. Calcd. for $\mathrm{C}_{23} \mathrm{H}_{18} \mathrm{ClN}_{3} \mathrm{O}_{4}$ : C 63.38, H 4.16, N 9.64. Found: C 63.21, H 4.03, N 9.58\%.

Methyl-4-[7-(4-chlorophenyl)3-methyl-5-oxo-4,5,6,7,8,9-hexahydroisoxazolo[5,4-b]quinolin4-yl)benzoate (4y). Colorless solid; ${ }^{1} \mathrm{H}$ NMR (200 MHz, DMSO- $\left.d_{6}\right) \delta 10.91(\mathrm{~s}, 1 \mathrm{NH}), 7.81-7.25$ $(\mathrm{m}, 8 \mathrm{H}), 5.06(5.11)(\mathrm{s}, 1 \mathrm{H}), 3.80(3.89)(\mathrm{s}, 3 \mathrm{H}), 3.42(\mathrm{~m}, 1 \mathrm{H}), 3.03-2.94(\mathrm{~m}, 2 \mathrm{H}), 2.78-2.69(\mathrm{~m}$, $2 \mathrm{H}), 1.83(1.76)(\mathrm{s}, 3 \mathrm{H}) ;{ }^{13} \mathrm{C}$ NMR $\left(100 \mathrm{MHz}, \mathrm{DMSO}-d_{6}\right) \delta$ 195.40, 165.90, 159.80, 151.70, 146.87, 144.80, 144.02, 131.12, 129.01, 127.08, 125.02, 110.05, 95.03, 52.05, 41.05, 37.93, 
37.03, 31,01, 11.05 ppm. MS (EI, $70 \mathrm{eV}): m / z(\%) 450$ (1.1), 448 (3.5) [M+], 338 (99.9), 244 (23.8), 172 (14.3), 77 (16.5). Anal. Calcd. for $\mathrm{C}_{25} \mathrm{H}_{21} \mathrm{ClN}_{2} \mathrm{O}_{4}$ : C 66.89, H 4.72, N 6.24. Found: C 66.64, H 4.39, N 6.11\%.

\section{7-(4-Chlorophenyl)-4-[4-(dimethylamino)phenyl]-3-methyl-4,7,8,9-tetrahydroisoxazolo-} [5,4-b]quinolin-5(6H)-one (4z). Colorless solid, mp 254-255 ${ }^{\circ} \mathrm{C} .{ }^{1} \mathrm{H}$ NMR (200 MHz, DMSO$\left.d_{6}\right) \delta 10.63(\mathrm{~s}, 1 \mathrm{NH}), 7.30-6.52(\mathrm{~m}, 8 \mathrm{H}), 4.84(\mathrm{~s}, 1 \mathrm{H}), 3.43(\mathrm{~m}, 1 \mathrm{H}), 3.01-2.65(\mathrm{~m}, 2 \mathrm{H}), 2.80(\mathrm{~s}$, $6 \mathrm{H}), 2.55-2.42(\mathrm{~m}, 2 \mathrm{H}), 1.86(\mathrm{~s}, 3 \mathrm{H}) \mathrm{ppm} ;{ }^{13} \mathrm{C}$ NMR (100 MHz, DMSO-d 6 ) $\delta 193.82,159.34$, $158.90,150.67,149.25,142.68,135.01,131.62,129.39$, 128.84, 112.55, 11.82, 96.57, 44.23,

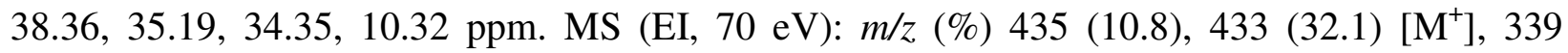
(22.6), 227 (17.8), 203 (99.9), 154 (38.9), 77 (18.9). Anal. Calcd. for $\mathrm{C}_{25} \mathrm{H}_{24} \mathrm{ClN}_{3} \mathrm{O}_{2}$ : C 69.20, $\mathrm{H}$ 5.57, N 9.68. Found: C 69.01, H 5.30, N 9.53\%.

3-Methyl-4-(4-(3-methyl-4,5,6,7,8,9-hexahydroisoxazolo[5,4-b]quinolin-5-one-4-yl)phenyl)4,5,6,7,8,9-hexahydroisoxazolo $[5,4-b]$ quinolin-5-one (6a). Colorless solid, mp 260-261 ${ }^{\circ} \mathrm{C} .{ }^{1} \mathrm{H}$ NMR (200 MHz, DMSO-d $) \delta 10.67(\mathrm{~s}, 2 \mathrm{NH}), 7.02(\mathrm{~m}, 4 \mathrm{H}), 4.89$ (s, 2H), 2.60-2.53 (m, 4H), 2.23-2.12 (m, 4H),1.83-1.72 (m, 4H), $1.78(\mathrm{~s}, 6 \mathrm{H}) \mathrm{ppm} ;{ }^{13} \mathrm{C}$ NMR (100 MHz, DMSO- $\left.d_{6}\right) \delta$ 195.01, 159.46, 158.76, 152.79, 144.79, 127.68, 111.46, 96.38, 37.35, 35.74, 27.63, 21.30, 10.26 ppm. MS (EI, $70 \mathrm{eV}): m / z(\%) 480$ (2.6) $\left[\mathrm{M}^{+}\right], 279$ (33.0), 205 (59.9), 203 (99.9), 188 (17.0), 112 (13.7), 84 (23.2). Anal. Calcd. for $\mathrm{C}_{28} \mathrm{H}_{26} \mathrm{~N}_{4} \mathrm{O}_{4}$ : C 69.70, H 5.43, N 11.61. Found: C 69.37, H $5.00, \mathrm{~N} 11.49 \%$.

3,7,7-Trimethyl-4-(4-(3,7,7-trimethyl-4,5,6,7,8,9-hexahydroisoxazolo[5,4-b]quinolin-5-one4-yl)phenyl)-4,5,6,7,8,9-hexahydroisoxazolo[5,4-b]quinolin-5-one (6b). Colorless solid, $\mathrm{mp}$ 266-267 ${ }^{\circ} \mathrm{C}$. ${ }^{1} \mathrm{H}$ NMR (200 MHz, DMSO-d $d_{6} \delta 10.64$ (s, 2NH), 7.00 (m, 4H), 4.87 (s, 2H), 2.50 $(\mathrm{d}, J 17.0 \mathrm{~Hz}, 2 \mathrm{H}), 2.33(\mathrm{~d}, J 17.0 \mathrm{~Hz}, 2 \mathrm{H}), 2.15(\mathrm{~d}, J 16.0 \mathrm{~Hz}, 2 \mathrm{H}), 1.96(\mathrm{~d}, J 16.0 \mathrm{~Hz}, 2 \mathrm{H}), 1.76$ $(\mathrm{s}, 6 \mathrm{H}), 0.98$ (s, 6H), 0.94 (s, 3H), 0.89 (s, 3H) ppm; ${ }^{13} \mathrm{C}$ NMR (100 MHz, DMSO-d $) \delta 194.63$, 159.58, 158.77, 150.63, 144.77, 127.60, 110.35, 96.38, 50.88, 35.76, 32.63, 29.02, 27.72, 21.17, 10.24 ppm MS (EI, $70 \mathrm{eV}): \mathrm{m} / z(\%) 536(36.6)\left[\mathrm{M}^{+}\right], 308$ (56.6), 232 (99.9), 122 (27.9), 84 (13.6). Anal. Calcd. for $\mathrm{C}_{32} \mathrm{H}_{34} \mathrm{~N}_{4} \mathrm{O}_{4}$ : C 71.36, H 6.36, N 10.40. Found: C 71.02, H 6.03, N $10.26 \%$.

Spiro[(3-methyl-7,8-dihydroisoxazolo[5,4-b]quinoline)-4,3' -(indole-2'(1'H)-one)]$\mathbf{5 ( 4 H , 6 H , 9 H )}$-one (8a). Colorless solid, mp 256-257 ${ }^{\circ} \mathrm{C} .{ }^{1} \mathrm{H}$ NMR $\left(200 \mathrm{MHz}, \mathrm{DMSO}-d_{6}\right) \delta 11.08$ $(\mathrm{s}, 1 \mathrm{NH}), 10.40(\mathrm{~s}, 1 \mathrm{NH}), 7.11-6.75(\mathrm{~m}, 4 \mathrm{H}), 2.64-2.57(\mathrm{~m}, 2 \mathrm{H}), 2.19-2.06(\mathrm{~m}, 2 \mathrm{H}), 1.93-1.76$ $(\mathrm{m}, 2 \mathrm{H}), 1.51(\mathrm{~s}, 3 \mathrm{H}) \mathrm{ppm} ;{ }^{13} \mathrm{C}$ NMR $\left(100 \mathrm{MHz}, \mathrm{DMSO}-d_{6}\right) \delta 191.03,162.53,155.21,147.08$, 135.40, 131.32, 119.08, 118.02, 110.53, 109.09, 98.06, 56.88, 54.26, 35.87, 28.04, 20.52, 11.02

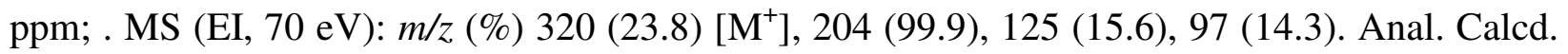
for $\mathrm{C}_{18} \mathrm{H}_{15} \mathrm{~N}_{3} \mathrm{O}_{3}$ : C 67.28, H 4.71, N 13.08. Found: C 67.08, H 4.43, N 12.99\%.

Spiro[(3,7,7-trimethyl-7,8-dihydroisoxazolo[5,4-b]quinoline)-4,3'-(indole-2'(1'H)-one)]$\mathbf{5 ( 4 H , 6 H , 9 H ) - o n e ~ ( 8 b ) . ~ C o l o r l e s s ~ s o l i d , ~ m p ~ 2 7 1 - 2 7 2 ~}{ }^{\circ} \mathrm{C}$. $1 \mathrm{H}$ NMR (200 MHz, DMSO- $\left.d_{6}\right) \delta{ }^{1} \mathrm{H}$ NMR (200 MHz, DMSO-d $) \delta 11.09(\mathrm{~s}, 1 \mathrm{NH}), 10.39(\mathrm{~s}, 1 \mathrm{NH}), 7.12-6.75(\mathrm{~m}, 4 \mathrm{H}), 2.56(\mathrm{~d}, J$ $17.0 \mathrm{~Hz}, 1 \mathrm{H}), 2.44$ (d, J 17.0 Hz, 1H), 2.11 (d, J 16.1 Hz, 1H), $1.96(\mathrm{~d}, J 16.1 \mathrm{~Hz}, 1 \mathrm{H}), 1.52$ (s, 3H), 1.01 (s, 3H), 0.98 (s, 3H) ppm; ${ }^{13} \mathrm{C}$ NMR (100 MHz, DMSO-d $) \delta 196.02,162.08,154.51$, 
147.03, 136.08, 130.02, 119.11, 118.32, 110.86, 109.02, 98.88, 56.89, 53.01, 52.81, 38.78, 31.16, 28.05, 27.14, 11.09 ppm. MS (EI, $70 \mathrm{eV}): m / z$ (\%) 350 (5.5) $\left[\mathrm{M}^{+}\right], 267$ (99.9), 265 (47.3), 175 (11.3), 77 (21.8). Anal. Calcd. for $\mathrm{C}_{20} \mathrm{H}_{19} \mathrm{~N}_{3} \mathrm{O}_{3}$ : C 68.75, H 5.48, N 12.03. Found: C 68.51, H $5.15, \mathrm{~N} 11.90 \%$.

Spiro[7-(4-chlorophenyl)-(3-methyl-7,8-dihydroisoxazolo[5,4-b]quinoline)-4,3'-(indole2'(1'H)-one)]-5(4H,6H,9H)-one (8c). Colorless solid, mp 264-265 ${ }^{\circ} \mathrm{C} .{ }^{1} \mathrm{H}$ NMR (200 MHz, DMSO-d $\left.{ }_{6}\right) \delta 11.23(\mathrm{~s}, 1 \mathrm{NH}), 10.43(\mathrm{~s}, 1 \mathrm{NH}), 7.40-6.79(\mathrm{~m}, 8 \mathrm{H}), 3.43(\mathrm{~m}, 1 \mathrm{H}), 3.06-2.70(\mathrm{~m}$, 2H), 2.59-2.29 (m, 2H), $1.53(\mathrm{~s}, 3 \mathrm{H}) \mathrm{ppm} ;{ }^{13} \mathrm{C}$ NMR (100 MHz, DMSO-d 6 ) $\delta 191.86,161.13$, $155.05,146.89,144.15,133.20,130.05,127.13,118.65,110.03,109.09$, 98.02, 56.32, 54.03, 42.04, 37.04, 31.91, 11.52 ppm. MS (EI, $70 \mathrm{eV}): \mathrm{m} / \mathrm{z}$ (\%) 431 (6.8) [ $\left.\mathrm{M}^{+}\right], 333$ (29.2), 251 (22.2), 183 (14.8), 128 (19.7), 93 (99.9), 77 (11.9). Anal. Calcd. for $\mathrm{C}_{24} \mathrm{H}_{18} \mathrm{ClN}_{3} \mathrm{O}_{3}: \mathrm{C}$ 66.75, $\mathrm{H} 4.20, \mathrm{~N}$ 9.73. Found: C 66.51, H 3.87, N 9.59\%.

General procedure for synthesis of 10a-o. A mixture of 5-amino-3-methylisoxazole 1 (1 mmol), aldehydes 9a-e ( $1 \mathrm{mmol})$, and cyclic $\beta$-di-ketones 3a-c $(1 \mathrm{mmol})$ in DMF $(2 \mathrm{~mL})$ in the presence of catalytic amounts of $\mathrm{HCl}$ or $\mathrm{Yb}(\mathrm{OTf})_{3}(5 \mathrm{~mol} \%)$ was heated to reflux for $5 \mathrm{~min}$ in a roundbottom flask equipped with a condenser. Then after cooling acetone $(10 \mathrm{~mL})$ was added and the precipitate formed was filtered out to give the solid compounds, which were washed with acetone and air-dried.

4-(2-Hydroxyphenyl)-3-methyl-4,7,8,9-tetrahydroisoxazolo[5,4-b]quinolin-5(6H)-one (10a). Colorless solid, mp 243-244 ${ }^{\circ} \mathrm{C} .{ }^{1} \mathrm{H}$ NMR (200 MHz, DMSO-d $) \delta 10.67(\mathrm{~s}, 1 \mathrm{NH}), 9.38(\mathrm{~s}, 1 \mathrm{H})$ 7.23-6.70 (m, 4H), $5.21(\mathrm{~s}, 1 \mathrm{H}), 2.57(\mathrm{~m}, 2 \mathrm{H}), 2.18(\mathrm{~m}, 2 \mathrm{H}), 1.88(\mathrm{~m}, 2 \mathrm{H}), 1.86(\mathrm{~s}, 3 \mathrm{H}) \mathrm{ppm} ;{ }^{13} \mathrm{C}$ NMR (100 MHz, DMSO- $\left.d_{6}\right) \delta 193.42,161.42,159.81,152.26,146.13,128.44,129.03,126.03$, 119.78, 107.02, 97.99, 37.97, 34.48, 27.97, 21.61, 11.08 ppm. MS (EI, $70 \mathrm{eV}): \mathrm{m} / z$ (\%) $296(3.5)$ [M $\mathrm{M}^{+}$, 204 (15.6), 184 (21.5), 172 (99.9), 93 (25.5), 77 (17.7). Anal. Calcd. for $\mathrm{C}_{17} \mathrm{H}_{16} \mathrm{~N}_{2} \mathrm{O}_{3}$ : C 68.91, H 5.44, N 9.45. Found: C 68.67, H 5.17, N 9.31\%.

4-(5-Chloro-2-hydroxyphenyl)-3-methyl-4,7,8,9-tetrahydroisoxazolo[5,4-b]quinolin-5(6H)one (10b). Colorless solid, mp 174-175 ${ }^{\circ} \mathrm{C} .{ }^{1} \mathrm{H}$ NMR $\left(200 \mathrm{MHz}, \mathrm{DMSO}-d_{6}\right) \delta 10.72(\mathrm{~s}, 1 \mathrm{NH})$, $8.87(\mathrm{~s}, 1 \mathrm{H}), 7.02-6.73(\mathrm{~m}, 3 \mathrm{H}), 5.20(\mathrm{~s}, 1 \mathrm{H}), 2.58(\mathrm{~m}, 2 \mathrm{H}), 2.18(\mathrm{~m}, 2 \mathrm{H}), 1.88(\mathrm{~m}, 2 \mathrm{H}), 1.87$ (s, $3 \mathrm{H}) \mathrm{ppm} ;{ }^{13} \mathrm{C}$ NMR $\left(100 \mathrm{MHz}, \mathrm{DMSO}-d_{6}\right) \delta 195.17,163.19,159.99,153.74,147.66,133.03$, $127.93,127.08,120.94,111.02,97.01,36.87,35.68,27.11,20.85,9.79$ ppm. MS (EI, $70 \mathrm{eV})$ : $\mathrm{m} / \mathrm{z}(\%) 330(2.8)\left[\mathrm{M}^{+}\right], 260$ (18.8), 236 (19.9), 203 (15.1), 132 (35.6), 127 (99.9), 77 (8.8). Anal. Calcd. for $\mathrm{C}_{17} \mathrm{H}_{15} \mathrm{ClN}_{2} \mathrm{O}_{3}$ : C 61.73, $\mathrm{H}$ 4.57, N 8.47. Found: C 61.50, H 4.14, N 8.35\%.

4-(5-Bromo-2-hydroxyphenyl)-3-methyl-4,7,8,9-tetrahydroisoxazolo[5,4-b]quinolin-5(6H)one (10c). Colorless solid, mp 218-219 ${ }^{\circ} \mathrm{C} .{ }^{1} \mathrm{H}$ NMR $\left(200 \mathrm{MHz}, \mathrm{DMSO}-d_{6}\right) \delta 10.72(\mathrm{~s}, 1 \mathrm{NH})$, 8.85 (s, 1H), 7.14-6.66 (m, 3H), $5.19(\mathrm{~s}, 1 \mathrm{H}), 2.64(\mathrm{~m}, 2 \mathrm{H}), 2.26(\mathrm{~m}, 2 \mathrm{H}), 1.92(\mathrm{~m}, 2 \mathrm{H}), 1.87$ (s, $3 \mathrm{H}) \mathrm{ppm} ;{ }^{13} \mathrm{C}$ NMR $\left(100 \mathrm{MHz}, \mathrm{DMSO}-d_{6}\right) \delta 194.09,160.88,158.31,152.26,145.45,130.02$, $128.17,125.62,120.13,110.18,95.74,36.12,34.98,27.85,21.88,10.78$ ppm. MS (EI, $70 \mathrm{eV})$ : $\mathrm{m} / \mathrm{z}(\%) 375$ (15.3) $\left[\mathrm{M}^{+}\right], 172$ (99.9), 280 (14.5), 203 (44.8), 172 (19.5), 77 (8.3). Anal. Calcd. for $\mathrm{C}_{17} \mathrm{H}_{15} \mathrm{BrN}_{2} \mathrm{O}_{3}$ : C 54.42, H 4.03, N 7.47. Found: C 54.20, H 3.75, N 7.34\%. 
4-(2-Hydroxy-3-methoxyphenyl)-3-methyl-4,7,8,9-tetrahydroisoxazolo[5,4- $b]$ quinolin5(6H)-one (10d). Colorless solid, mp 239-240 ${ }^{\circ} \mathrm{C} .{ }^{1} \mathrm{H}$ NMR (200 MHz, DMSO- $\left.d_{6}\right) \delta 10.68$ (s, $1 \mathrm{NH}), 8.67(\mathrm{~s}, 1 \mathrm{H}), 6.68 .-6.44(\mathrm{~m}, 3 \mathrm{H}), 5.24(\mathrm{~s}, 1 \mathrm{H}), 3.73(\mathrm{~s}, 3 \mathrm{H}), 2.55(\mathrm{~m}, 2 \mathrm{H}), 2.18(\mathrm{~m}, 2 \mathrm{H})$, $1.88(\mathrm{~m}, 2 \mathrm{H}), 1.85(\mathrm{~s}, 3 \mathrm{H}) \mathrm{ppm} ;{ }^{13} \mathrm{C}$ NMR (100 MHz, DMSO-d 6 ). $\delta$ 195.42, 159.02, 153.31, $149.26,146.66,128.02$, 127.93, 127.44, 127.11, 125.94, 111.02, 95.68, 55.34, 37.55, 34.77, 27.11, 20.89, 10.90 ppm. MS (EI, $70 \mathrm{eV}): \mathrm{m} / z$ (\%) 326 (48.9) [ $\left.\mathrm{M}^{+}\right], 230$ (22.8), 203 (54.8), 172 (99.9), 123 (14.3). Anal. Calcd. for $\mathrm{C}_{18} \mathrm{H}_{18} \mathrm{~N}_{2} \mathrm{O}_{4}$ : C 66.25, H 5.56, N 8.58. Found: C 66.03, H 5.24, N $8.50 \%$.

4-(2-Hydroxy-5-nitrophenyl)-3-methyl-4,7,8,9-tetrahydroisoxazolo[5,4-b]quinolin-5(6H)one (10e). Colorless solid, mp 248-249 ${ }^{\circ} \mathrm{C} .{ }^{1} \mathrm{H}$ NMR $\left(200 \mathrm{MHz}, \mathrm{DMSO}-d_{6}\right) \delta 11.14$ (s, 1OH), $10.82(\mathrm{~s}, 1 \mathrm{NH}), 7.90-6.86(\mathrm{~m}, 3 \mathrm{H}), 5.29(\mathrm{~s}, 1 \mathrm{H}), 2.59(\mathrm{~m}, 2 \mathrm{H}), 2.18(\mathrm{~m}, 2 \mathrm{H}), 1.90(\mathrm{~m}, 2 \mathrm{H}), 1.87$ $(\mathrm{s}, 3 \mathrm{H}) \mathrm{ppm} ;{ }^{13} \mathrm{C}$ NMR $\left(100 \mathrm{MHz}, \mathrm{DMSO}-d_{6}\right) \delta 195.11,162.88,160.33,150.79,146.09,140.99$, 129.03, 127.81, 121.62, 115.91, 110.67, 97.77, 36.93, 35.41, 28.11, 20.99, 11.01 ppm. MS (EI, $70 \mathrm{eV}): \mathrm{m} / z$ (\%) $341(8.5)\left[\mathrm{M}^{+}\right], 285$ (15.5), 247 (22.5), 203 (99.9), 172 (15.6), 139 (28.7). Anal. Calcd. for $\mathrm{C}_{17} \mathrm{H}_{15} \mathrm{~N}_{3} \mathrm{O}_{5}$ : C 59.82, H 4.43, N 12.31. Found: C 59.60, H 4.15, N 12.19\%.

4-(2-Hydroxyphenyl)-3,7,7-trimethyl-4,7,8,9-tetrahydroisoxazolo[5,4-b]quinolin-5(6H)-one (10f). Colorless solid, mp 248-249 ${ }^{\circ} \mathrm{C} .{ }^{1} \mathrm{H}$ NMR (200 MHz, DMSO- $\left.d_{6}\right) \delta 10.69$ (s, 1OH), 9.12 (s, $1 \mathrm{NH}), 7.28-6.70(\mathrm{~m}, 4 \mathrm{H}), 5.18(\mathrm{~s}, 1 \mathrm{H}), 2.52(\mathrm{~d}, J 17.1 \mathrm{~Hz}, 1 \mathrm{H}), 2.35(\mathrm{~d}, J 17.1 \mathrm{~Hz}, 1 \mathrm{H}), 2.16(\mathrm{~d}$, $J 16.1 \mathrm{~Hz}, 1 \mathrm{H}), 1.98(\mathrm{~d}, J 16.1 \mathrm{~Hz}, 1 \mathrm{H}), 1.88(\mathrm{~s}, 3 \mathrm{H}), 1.00(\mathrm{~s}, 3 \mathrm{H}), 0.97(\mathrm{~s}, 3 \mathrm{H}) \mathrm{ppm} ;{ }^{13} \mathrm{C} \mathrm{NMR}$ $\left(100 \mathrm{MHz}, \mathrm{DMSO}-d_{6}\right) \delta 195.13,159.19,158.11,155.37,151.77,136.04,131.83,129.99,118.00$, 110.60, 109.59, 107.36, 96.85, 51.84, 41.91, 31.62, 30.76, 29.64, 27.81, 10.97 ppm. MS (EI, 70 $\mathrm{eV}): m / z(\%) 324$ (3.2) [ $\left.\mathrm{M}^{+}\right], 230$ (99.9), 228 (16.8), 202 (48.7), 122 (14.9), 93 (24.2). Anal. Calcd. for $\mathrm{C}_{19} \mathrm{H}_{20} \mathrm{~N}_{2} \mathrm{O}_{3}:$ C 70.35, H 6.21, N 8.64. Found: C 70.13, H 6.00, N 8.56\%.

4-(5-Chloro-2-hydroxyphenyl)-3,7,7-trimethyl-4,7,8,9-tetrahydroisoxazolo[5,4-b]quinolin5(6H)-one (10g). Colorless solid, mp 257-258 ${ }^{\circ} \mathrm{C} .{ }^{1} \mathrm{H}$ NMR $\left(200 \mathrm{MHz}, \mathrm{DMSO}-d_{6}\right) \delta 10.68(\mathrm{~s}$, $1 \mathrm{OH}), 9.69(\mathrm{~s}, 1 \mathrm{NH}), 6.96-6.70(\mathrm{~m}, 3 \mathrm{H}), 5.17$ (s, 1H), 2.50 (d, J 16.9 Hz, 1H), 2.39 (d, J 16.9 Hz, 1H), 2.19 (d, J 16.1 Hz, 1H), 1.97 (d, J 16.1 Hz, 1H), 1.88 (s, 3H), 1.00 (s, 3H), 0.97 (s, 3H) ppm; ${ }^{13} \mathrm{C}$ NMR (100 MHz, DMSO- $\left.d_{6}\right) \delta 194.64,159.95,158.74,135.49,128.93,127.10,122.91$, $117.44,109.56,95.76,50.85,40.91,32.63,32.11,29.22,27.13,10.22$ ppm. MS (EI, $70 \mathrm{eV}): \mathrm{m} / z$ (\%) 358 (23.7) $\left[\mathrm{M}^{+}\right], 262$ (36.5), 231 (14.3), 170 (19.3), 127 (99.9). Anal. Calcd. for $\mathrm{C}_{19} \mathrm{H}_{19} \mathrm{ClN}_{2} \mathrm{O}_{3}$ : C 63.60, H 5.34, N 7.81. Found: C 63.38, H 5.01, N 7.71\%.

4-(5-Bromo-2-hydroxyphenyl)-3,7,7-trimethyl-4,7,8,9-tetrahydroisoxazolo[5,4- $b$ ]quinolin5(6H)-one (10h). Colorless solid, mp 265-266 ${ }^{\circ} \mathrm{C} .{ }^{1} \mathrm{H}$ NMR $\left(200 \mathrm{MHz}, \mathrm{DMSO}-d_{6}\right) \delta 10.66(\mathrm{~s}$, $1 \mathrm{OH}), 9.72(\mathrm{~s}, 1 \mathrm{NH}), 7.08-6.65(\mathrm{~m}, 3 \mathrm{H}), 5.16(\mathrm{~s}, 1 \mathrm{H}), 2.52$ (d, J 17.0 Hz, 1H), 2.35 (d, J $17.0 \mathrm{~Hz}$, $1 \mathrm{H}), 2.16(\mathrm{~d}, J 16.1 \mathrm{~Hz}, 1 \mathrm{H}), 1.98(\mathrm{~d}, J 16.1 \mathrm{~Hz}, 1 \mathrm{H}), 1.88(\mathrm{~s}, 3 \mathrm{H}), 1.00(\mathrm{~s}, 3 \mathrm{H}), 0.97$ (s, 3H) ppm;

${ }^{13} \mathrm{C}$ NMR (100 MHz, DMSO- $\left.d_{6}\right) \delta 194.63,159.92,158.75,153.64,151.77,136.04,131.83$, 129.99, 118.00, 110.60, 109.59, 107.36, 95.79, 50.84, 40.90, 32.62, 30.06, 29.31,27.02, 10.23 ppm. MS (EI, $70 \mathrm{eV}): m / z$ (\%) 404 (22.9) [ $\left.\mathrm{M}^{+}\right], 403$ (11.1), 387 (26.8), 344 (55.8), 307 (23.1), 279 (22.0), 233 (60.7), 127 (21.6), 63 (43.0) 62 (99.9). Anal. Calcd. for $\mathrm{C}_{19} \mathrm{H}_{19} \mathrm{BrN}_{2} \mathrm{O}_{3}$ : C 56.59, H 4.75, N 6.95. Found: C 56.42, H 4.44, N 6.83\%. 
4-(2-Hydroxy-3-methoxyphenyl)-3,7,7-trimethyl-4,7,8,9-tetrahydroisoxazolo[5,4-b]quinolin-5(6H)-one (10i). Colorless solid, mp 238-239 ${ }^{\circ} \mathrm{C} .{ }^{1} \mathrm{H}$ NMR (200 MHz, DMSO- $\left.d_{6}\right) \delta 10.71$ (s, $1 \mathrm{OH}), 8.61(\mathrm{~s}, 1 \mathrm{NH}), 6.67-6.48(\mathrm{~m}, 3 \mathrm{H}), 5.23(\mathrm{~s}, 1 \mathrm{H}), 3.72(\mathrm{~s}, 3 \mathrm{H}), 2.52(\mathrm{~d}, J 16.9 \mathrm{~Hz}, 1 \mathrm{H}), 2.37$ $(\mathrm{d}, J 16.9 \mathrm{~Hz}, 1 \mathrm{H}), 2.17(\mathrm{~d}, J 16.1 \mathrm{~Hz}, 1 \mathrm{H}), 1.95(\mathrm{~d}, J 16.1 \mathrm{~Hz}, 1 \mathrm{H}), 1.87$ (s, 3H), $1.00(\mathrm{~s}, 3 \mathrm{H})$, 0.95 (s, 3H) ppm; ${ }^{13} \mathrm{C}$ NMR (100 MHz, DMSO- $\left.d_{6}\right) \delta$ 194.95, 159.87, 158.76, 151.68, 147.90, 143.06, 133.90, 121.18, 119.24, 110.24, 109.79, 96.41, 56.15, 50.88, 40.91, 32.55, 29.30, 29.19, 27.27, 10.70 ppm. MS (EI, $70 \mathrm{eV}): \mathrm{m} / \mathrm{z}$ (\%) 354 (23.0) $\left[\mathrm{M}^{+}\right], 258$ (15.4), 231 (99.9), 137 (33.5), 151 (43), 96 (45.5). Anal. Calcd. for $\mathrm{C}_{20} \mathrm{H}_{22} \mathrm{~N}_{2} \mathrm{O}_{4}$ : C 67.78, $\mathrm{H}$ 6.26, N 7.90. Found: C 67.56, H $6.01, \mathrm{~N} 7.81 \%$.

4-(2-Hydroxy-5-nitrophenyl)-3,7,7-trimethyl-4,7,8,9-tetrahydroisoxazolo[5,4-b]quinolin5(6H)-one (10j). Colorless solid, mp 263-264 ${ }^{\circ} \mathrm{C} .{ }^{1} \mathrm{H}$ NMR $\left(200 \mathrm{MHz}, \mathrm{DMSO}-d_{6}\right) \delta 11.15$ (s, $1 \mathrm{OH}), 10.78(\mathrm{~s}, 1 \mathrm{NH}), 7.90-6.88(\mathrm{~m}, 3 \mathrm{H}), 5.27(\mathrm{~s}, 1 \mathrm{H}), 2.58(\mathrm{~d}, J 17.0 \mathrm{~Hz}, 1 \mathrm{H}), 2.36(\mathrm{~d}, J 17.0$ $\mathrm{Hz}, 1 \mathrm{H}), 2.18(\mathrm{~d}, J 16.1 \mathrm{~Hz}, 1 \mathrm{H}), 1.97$ (d, J 16.1 Hz, 1H), 1.87 (s, 3H), 1.00 (s, 3H), 0.95 (s, 3H) ppm; ${ }^{13} \mathrm{C}$ NMR (100 MHz, DMSO- $\left.d_{6}\right) \delta 194.68,161.11,160.38,158.80,152.16,140.59,134.70$, 125.61, 124.13, 116.41, 109.71, 95.56, 51.14, 41.32, 32.73, 30.69, 29.47, 27.21, 10.32 ppm. MS

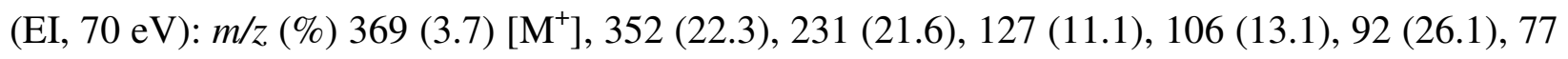
(35.8), 63 (49.7), 42 (99.9). Anal. Calcd. for $\mathrm{C}_{19} \mathrm{H}_{19} \mathrm{~N}_{3} \mathrm{O}_{5}$ : C 61.78, H 5.18, N 11.38. Found: C $61.55, \mathrm{H} 4.89, \mathrm{~N} 11.27 \%$.

7-(4-Chlorophenyl)-4-(2-hydroxyphenyl)-3-methyl-4,7,8,9-tetrahydroisoxazolo[5,4-b]quinolin-5(6H)-one (10k). Colorless solid; ${ }^{1} \mathrm{H}$ NMR (200 MHz, DMSO-d $) \delta 10.90(\mathrm{~s}, 1 \mathrm{NH})$, 9.04(9.01) (s, 1OH), 6.95-6.42 (m, 8H), 5.03(5.01) (s, 1H), $3.51(\mathrm{~m}, 1 \mathrm{H}), 2.94(\mathrm{~m}, 2 \mathrm{H}), 2.66(\mathrm{~m}$, 2H), 2.24 (s, 3H) ppm; ${ }^{13} \mathrm{C}$ NMR (100 MHz, DMSO-d $) \delta 195.45,161.80,153.76,147.96$, $147.07,146.03,131.16,127.68,122.25,118.06,111.25,108.04,95.54,54.99,43.48,38.19$, 32.72, 32.10, 11.05 ppm. MS (EI, $70 \mathrm{eV}): \mathrm{m} / z$ (\%) 406 (3) $\left[\mathrm{M}^{+}\right], 313$ (99.9), 294 (15.6), 202 (65.7), 112 (14.3). Anal. Calcd. for $\mathrm{C}_{23} \mathrm{H}_{19} \mathrm{ClN}_{2} \mathrm{O}_{3}$ : C 67.90, H 4.71, N 6.89. Found: C 67.73, $\mathrm{H}$ $4.40, \mathrm{~N} 6.77 \%$.

4-(5-Chloro-2-hydroxyphenyl)-7-(4-chlorophenyl)-3-methyl-4,7,8,9-tetrahydroisoxazolo[5,4-b]quinolin-5(6H)-one (10I). Colorless solid; ${ }^{1} \mathrm{H}$ NMR (200 MHz, DMSO- $\left.d_{6}\right) \delta 11.11$ (s, $1 \mathrm{NH}), 8.88(8.75)(\mathrm{s}, 1 \mathrm{OH}), 7.50-7.00(\mathrm{~m}, 7 \mathrm{H}), 4.97(4.91)(\mathrm{s}, 1 \mathrm{H}), 3.51(\mathrm{~m}, 1 \mathrm{H}), 3.08(\mathrm{~m}, 2 \mathrm{H})$, $2.66(\mathrm{~m}, 2 \mathrm{H}), 2.40(2.39)(\mathrm{s}, 3 \mathrm{H}) \mathrm{ppm} ;{ }^{13} \mathrm{C}$ NMR (100 MHz, DMSO-d 6 ) $\delta$ 195.67, 166.66, $160.33,157.96,147.74,142.19,137.24,132.63,131.09,129.36,128.40,126.29,123.45,119.53$, 118.79, 95.61, 43.91, 42.57, 37.58, 34.50, 26.21, 11.01 ppm. MS (EI, $70 \mathrm{eV}): \mathrm{m} / z$ (\%) $440(3.8)$ $\left[\mathrm{M}^{+}\right], 329$ (99.9), 313 (32.3), 202 (18.9), 127 (16.5). Anal. Calcd. for $\mathrm{C}_{23} \mathrm{H}_{18} \mathrm{Cl}_{2} \mathrm{~N}_{2} \mathrm{O}_{3}$ : C 62.60, $\mathrm{H}$ 4.11, N 6.35. Found: C 62.38, H 3.83, N 6.27\%.

4-(5-Bromo-2-hydroxyphenyl)-7-(4-chlorophenyl)-3-methyl-4,7,8,9-tetrahydroisoxazolo[5,4-b]quinolin-5(6H)-one (10m). Colorless solid; ${ }^{1} \mathrm{H}$ NMR $\left(200 \mathrm{MHz}, \mathrm{DMSO}-d_{6}\right) \delta 11.02(\mathrm{~s}$, $1 \mathrm{NH}), 8.78(8.72)(\mathrm{s}, 1 \mathrm{OH}), 7.52-6.95(\mathrm{~m}, 7 \mathrm{H}), 4.97(4.90)(\mathrm{s}, 1 \mathrm{H}), 3.51(\mathrm{~m}, 1 \mathrm{H}), 2.94(\mathrm{~m}, 2 \mathrm{H})$, $2.54(\mathrm{~m}, 2 \mathrm{H}), 2.39(\mathrm{~s}, 3 \mathrm{H}) \mathrm{ppm} ;{ }^{13} \mathrm{C} \mathrm{NMR}\left(100 \mathrm{MHz}, \mathrm{DMSO}-d_{6}\right) \delta 195.69,165.66,159.33$, 157.96, 148.74, 142.09, 137.24, 132.63, 131.09, 129.36, 128.40, 126.19, 123.45, 119.53, 118.79, 95.61, 43.91, 42.57, 37.58, 34.50, 26.21, $10.97 \mathrm{ppm}$. MS (EI, $70 \mathrm{eV}): \mathrm{m} / \mathrm{z}(\%) 484(3.8)\left[\mathrm{M}^{+}\right]$, 
389 (99.9), 387 (77.5), 251 (16.7), 199 (12.0), 142 (31.2), 114 (10.0). Anal. Calcd. for $\mathrm{C}_{23} \mathrm{H}_{18} \mathrm{BrClN}_{2} \mathrm{O}_{3}$ : C 56.87, H 3.74, N 5.77. Found: C 56.69, H 3.45, N 5.68\%.

7-(4-Chlorophenyl)-4-(2-hydroxy-3-methoxyphenyl)-3-methyl-4,7,8,9-tetrahydroisoxazolo[5,4-b]quinolin-5(6H)-one (10n). Colorless solid; ${ }^{1} \mathrm{H}$ NMR $\left(200 \mathrm{MHz}, \mathrm{DMSO}-d_{6}\right) \delta 10.91$ (s, $1 \mathrm{NH}), 9.04(8.99)(\mathrm{s}, 1 \mathrm{OH}), 6.94-6.42(\mathrm{~m}, 7 \mathrm{H}), 5.00(4.94)(\mathrm{s}, 1 \mathrm{H}), 3.74(\mathrm{~s}, 3 \mathrm{H}), 3.51(\mathrm{~m}, 1 \mathrm{H})$, $2.94(\mathrm{~m}, 2 \mathrm{H}), 2.66(\mathrm{~m}, 2 \mathrm{H}), 2.24(\mathrm{~s}, 3 \mathrm{H}) \mathrm{ppm} ;{ }^{13} \mathrm{C}$ NMR (100 MHz, DMSO-d 6$) \delta 195.45$, $161.80,153.76,147.96,147.07,146.03,131.16,127.68,122.25,118.06,111.25,108.04,95.54$, 54.99, 43.48, 38.19, 32.72, 32.10, 11.05 ppm. MS (EI, $70 \mathrm{eV}): \mathrm{m} / \mathrm{z}(\%) 436(18.6)\left[\mathrm{M}^{+}\right], 438$ (5.6), 313 (99.9), 186 (43.5), 123 (15.5). Anal. Calcd. for $\mathrm{C}_{24} \mathrm{H}_{21} \mathrm{ClN}_{2} \mathrm{O}_{4}: \mathrm{C}$ 65.98, $\mathrm{H}$ 4.84, N 6.41. Found: C 65.77, H 4.55, N 6.30\%.

7-(4-Chlorophenyl)-4-(2-hydroxy-5-nitrophenyl)-3-methyl-4,7,8,9-tetrahydroisoxazolo[5,4-b]quinolin-5(6H)-one (10o). Colorless solid; ${ }^{1} \mathrm{H}$ NMR (200 MHz, DMSO- $\left.d_{6}\right) \delta 11.12$ (s, $1 \mathrm{NH}), 8.90(8.88)(\mathrm{s}, 1 \mathrm{OH}), 7.54-7.02(\mathrm{~m}, 7 \mathrm{H}), 4.99(4.96)(\mathrm{s}, 1 \mathrm{H}), 3.51(\mathrm{~m}, 1 \mathrm{H}), 3.07(\mathrm{~m}, 2 \mathrm{H})$, $2.66(\mathrm{~m}, 2 \mathrm{H}), 2.40(2.39)(\mathrm{s}, 3 \mathrm{H}) \mathrm{ppm} ;{ }^{13} \mathrm{C}$ NMR (100 MHz, DMSO-d 6 ) $\delta$ 195.42, 161.76, 152.66, 147.97, 147.17, 146.03, 131.16, 127.68, 122.25, 118.06, 111.25, 108.74, 95.74, 43.48, 38.19, 32.72, 32.10, 11.05 ppm. MS (EI, $70 \mathrm{eV}): \mathrm{m} / z$ (\%) 451 (11.4) $\left[\mathrm{M}^{+}\right], 340$ (18.6), 313 (99.9), 202 (12.2), 139 (18.4). 112 (21.2), 96 (24.5). Anal. Calcd. for $\mathrm{C}_{23} \mathrm{H}_{18} \mathrm{ClN}_{3} \mathrm{O}_{5}$ : C 61.14, $\mathrm{H}$ 4.02, N 9.30. Found: C 61.00, H 3.86, N 9.23\%.

General procedure for synthesis of 11a-o. A mixture of 5-amino-3-methylisoxazole 1 (1 mmol), aldehydes 9a-e $(1 \mathrm{mmol})$, and cyclic $\beta$-di-ketones 3a-c $(1 \mathrm{mmol})$ with a catalytic amount of $\mathrm{Et}_{3} \mathrm{~N}$ was ultrasonicated in ethanol $(10 \mathrm{~mL})$ at room temperature for $90 \mathrm{~min}$. Compounds 11a$\mathbf{o}$ were isolated from 12a-o in individual state by crystallization from ethyl acetate $(10 \mathrm{~mL})$.

9-(5-Amino-3-methylisoxazol-4-yl)-2,3,4,9-tetrahydro-1H-xanthen-1-one (11a). Colorless solid; mp 196-197 ${ }^{\circ} \mathrm{C} .{ }^{1} \mathrm{H}$ NMR (200 MHz, DMSO-d $) \delta$ 7.18-6.93 (m, 4H), $6.39\left(2, \mathrm{NH}_{2}\right), 4.78$ $(\mathrm{s}, 1 \mathrm{H}), 2.63(\mathrm{~m}, 2 \mathrm{H}), 2.27(\mathrm{~m}, 2 \mathrm{H}), 1.93(\mathrm{~m}, 2 \mathrm{H}), 1.62(\mathrm{~s}, 3 \mathrm{H}) \mathrm{ppm} ;{ }^{13} \mathrm{C} \mathrm{NMR}(100 \mathrm{MHz}$, DMSO- $\left.d_{6}\right) \delta 195.76,168.82,159.90,149.77,132.45,131.55,127.85,120.87,117.85,110.69$,

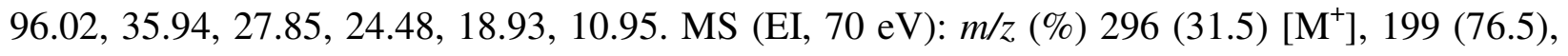
183 (16.8), 97 (99.9), 94 (19.9), 78 (22.6). Anal. Calcd. for $\mathrm{C}_{17} \mathrm{H}_{16} \mathrm{~N}_{2} \mathrm{O}_{3}: \mathrm{C}$ 68.91, H 5.44, N 9.45. Found: C 68.69, H 5.13, N 9.33\%.

9-(5-Amino-3-methylisoxazol-4-yl)-7-chloro-2,3,4,9-tetrahydro-1H-xanthen-1-one (11b). Colorless solid; mp 209-210 ${ }^{\circ} \mathrm{C} .{ }^{1} \mathrm{H}$ NMR (200 MHz, DMSO- $\left.d_{6}\right) \delta$ 7.29-7.12 (m, 3H), 6.47 (2, $\left.\mathrm{NH}_{2}\right), 4.83(\mathrm{~s}, 1 \mathrm{H}), 2.65(\mathrm{~m}, 2 \mathrm{H}), 2.29(\mathrm{~m}, 2 \mathrm{H}), 1.93(\mathrm{~m}, 2 \mathrm{H}), 1.68(\mathrm{~s}, 3 \mathrm{H}) \mathrm{ppm} ;{ }^{13} \mathrm{C}$ NMR $(100$ $\left.\mathrm{MHz}, \mathrm{DMSO}-d_{6}\right) \delta 193.64,170.72,159.56,149.97,135.45,131.80,128.37,120.03,118.95$,

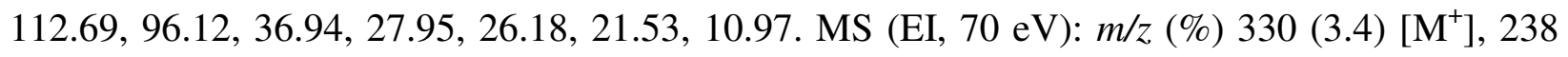
(19.9), 217 (99.9), 205 (56.8), 97 (13.0), 77 (16.5). Anal. Calcd. for $\mathrm{C}_{17} \mathrm{H}_{15} \mathrm{ClN}_{2} \mathrm{O}_{3}: \mathrm{C} 61.73, \mathrm{H}$ 4.57, N 8.47. Found: C 61.54, H 4.25, N 8.39\%.

9-(5-Amino-3-methylisoxazol-4-yl)-7-bromo-2,3,4,9-tetrahydro-1H-xanthen-1-one (11c). Colorless solid; mp 194-195 ${ }^{\circ} \mathrm{C} .{ }^{1} \mathrm{H}$ NMR (200 MHz, DMSO- $\left.d_{6}\right) \delta$ 7.38-7.07 (m, 3H), $6.54(2$, $\left.\mathrm{NH}_{2}\right), 4.83(\mathrm{~s}, 1 \mathrm{H}), 2.64(\mathrm{~m}, 2 \mathrm{H}), 2.32(\mathrm{~m}, 2 \mathrm{H}), 1.95(\mathrm{~m}, 2 \mathrm{H}), 1.66(\mathrm{~s}, 3 \mathrm{H}) \mathrm{ppm} ;{ }^{13} \mathrm{C}$ NMR $(100$ 
MHz, DMSO- $\left.d_{6}\right) \delta 196.64,166.72,159.02,148.67,132.45,131.24,127.27,118.87,116.85$, 111.59, 95.02, 36.94, 27.41, 25.18, 20.53, 10.47. MS (EI, $70 \mathrm{eV}): \mathrm{m} / z$ (\%) 375 (3.4) $\left[\mathrm{M}^{+}\right], 278$ (14.8), 207 (22.4), ), 182 (99.9), 97 (12.5), 77 (33.1). Anal. Calcd. for $\mathrm{C}_{17} \mathrm{H}_{15} \mathrm{BrN}_{2} \mathrm{O}_{3}$ : C 54.42, $\mathrm{H}$ 4.03, N 7.47. Found: C 54.24, H 3.71, N 7.37\%.

9-(5-Amino-3-methylisoxazol-4-yl)-5-methoxy-2,3,4,9-tetrahydro-1H-xanthen-1-one (11d). Colorless solid; mp 199-200 ${ }^{\circ} \mathrm{C} .{ }^{1} \mathrm{H}$ NMR (200 MHz, DMSO- $\left.d_{6}\right) \delta 6.99-6.52(\mathrm{~m}, 3 \mathrm{H}), 6.39(2$, $\left.\mathrm{NH}_{2}\right), 4.77(\mathrm{~s}, 1 \mathrm{H}), 3.80(\mathrm{~s}, 3 \mathrm{H}), 2.66(\mathrm{~m}, 2 \mathrm{H}), 2.27(\mathrm{~m}, 2 \mathrm{H}), 1.93(\mathrm{~m}, 2 \mathrm{H}), 1.64$ (s, 3H) ppm; ${ }^{13} \mathrm{C}$ NMR (100 MHz, DMSO-d $d_{6} \delta 193.10,170.98,167.95,159.80,155.65,130.92,128.04$, 120.25, 112.44, 111.65, 107.30, 99.89, 55.76, 37.61, 28.65, 25.62, 20.78, 11.01. MS (EI, $70 \mathrm{eV})$ : $\mathrm{m} / \mathrm{z}(\%) 326$ (9.8) $\left[\mathrm{M}^{+}\right], 257$ (33.7), 230 (99.9), 204 (45.6), 123 (18.7), 95 (14.3) Anal. Calcd. for $\mathrm{C}_{18} \mathrm{H}_{18} \mathrm{~N}_{2} \mathrm{O}_{4}$ : C 66.25, H 5.56, N 8.58. Found: C 66.03, H 5.24, N 8.50\%.

9-(5-Amino-3-methylisoxazol-4-yl)-7-nitro-2,3,4,9-tetrahydro-1H-xanthen-1-one

(11e). Colorless solid; mp 230-231 ${ }^{\circ} \mathrm{C} .{ }^{1} \mathrm{H}$ NMR (200 MHz, DMSO- $\left.d_{6}\right) \delta 8.10-7.37(\mathrm{~m}, 3 \mathrm{H}), 6.67(2$, $\left.\mathrm{NH}_{2}\right), 4.98(\mathrm{~s}, 1 \mathrm{H}), 2.71(\mathrm{~m}, 2 \mathrm{H}), 2.31(\mathrm{~m}, 2 \mathrm{H}), 1.97(\mathrm{~m}, 2 \mathrm{H}), 1.66(\mathrm{~s}, 3 \mathrm{H}) \mathrm{ppm} ;{ }^{13} \mathrm{C}$ NMR $(100$ MHz, DMSO- $\left.d_{6}\right) \delta 193.30,171.98,169.95,159.80,158.65,143.92,125.90,122.25,119.44$, 107.30, 99.89, 55.76, 37.61, 28.65, 25.62, 20.78, 11.01. MS (EI, $70 \mathrm{eV}): \mathrm{m} / z$ (\%) $341(3)\left[\mathrm{M}^{+}\right]$, 245 (99.9), 205 (14.4), 182 (34.4), 138 (18.2), 98 (19.9). Anal. Calcd. for $\mathrm{C}_{17} \mathrm{H}_{15} \mathrm{~N}_{3} \mathrm{O}_{5}$ : C 59.82, H 4.43, N 12.31. Found: C 59.64, H 4.11, N 12.20\%.

9-(5-Amino-3-methylisoxazol-4-yl)-3,3-dimethyl-2,3,4,9-tetrahydro-1H-xanthen-1-one (11f). Colorless solid; mp 126-127 ${ }^{\circ} \mathrm{C} .{ }^{1} \mathrm{H}$ NMR (200 MHz, DMSO- $\left.d_{6}\right) \delta$ 7.15-7.04 (m, 4H), $6.36(2$, $\left.\mathrm{NH}_{2}\right), 4.79(\mathrm{~s}, 1 \mathrm{H}), 2.56(\mathrm{~d}, J 17.0 \mathrm{~Hz}, 1 \mathrm{H}), 2.52$ (d, J $\left.17.0 \mathrm{~Hz}, 1 \mathrm{H}\right), 2.22(\mathrm{~s}, 2 \mathrm{H}), 1.61(\mathrm{~s}, 3 \mathrm{H})$, $1.03(\mathrm{~s}, 3 \mathrm{H}), 0.99$ (s, 3H) ppm; ${ }^{13} \mathrm{C}$ NMR (100 MHz, DMSO- $\left.d_{6}\right) \delta 191.10,170.18,167.95$, $157.80,152.85,130.12,127.04,122.11,119.25,115.44,112.65,107.30,99.89,51.84,33.61$,

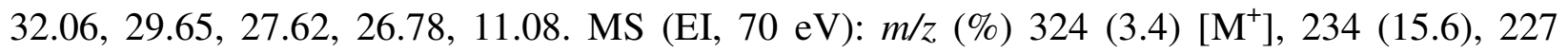
(68.5), 97 (99.9), 93 (13.0). Anal. Calcd. for $\mathrm{C}_{19} \mathrm{H}_{20} \mathrm{~N}_{2} \mathrm{O}_{3}$ : C 70.35, H 6.21, N 8.64. Found: C 70.13, H 5.92, N 8.55\%.

9-(5-Amino-3-methylisoxazol-4-yl)-7-chloro-3,3-dimethyl-2,3,4,9-tetrahydro-1H-xanthen-1one (11g). Colorless solid; mp 217-218 ${ }^{\circ} \mathrm{C} .{ }^{1} \mathrm{H}$ NMR (200 MHz, DMSO- $\left.d_{6}\right) \delta$ 7.38-7.08 (m, 3H), $6.40\left(2, \mathrm{NH}_{2}\right), 4.82(\mathrm{~s}, 1 \mathrm{H}), 2.57(\mathrm{~d}, J 17.0 \mathrm{~Hz}, 1 \mathrm{H}), 2.50(\mathrm{~d}, J 17.0 \mathrm{~Hz}, 1 \mathrm{H}), 2.22(\mathrm{~s}, 2 \mathrm{H}), 1.66$ (s, $3 \mathrm{H}), 1.02$ (s, 3H), 0.99 (s, 3H) ppm; ${ }^{13} \mathrm{C}$ NMR (100 MHz, DMSO-d $) \delta 194.12,165.98,160.95$, $152.80,136.85,130.12,128.04,119.25,115.04,112.60,109.30,97.89,51.84,33.61,32.06$, 29.65, 27.62, 26.91, 10.98. MS (EI, $70 \mathrm{eV}): \mathrm{m} / z$ (\%) 360 (6.7) $\left[\mathrm{M}^{+}\right], 263$ (99.9), 238 (34.8), 228 (24.7), 97 (45.0). Anal. Calcd. for $\mathrm{C}_{19} \mathrm{H}_{19} \mathrm{ClN}_{2} \mathrm{O}_{3}$ : C 63.60, H 5.34, N 7.81. Found: C 63.37, H $5.02, \mathrm{~N} 7.72 \%$.

9-(5-Amino-3-methylisoxazol-4-yl)-7-bromo-3,3-dimethyl-2,3,4,9-tetrahydro-1H-xanthen-1one (11h). Colorless solid; mp 223-224 ${ }^{\circ} \mathrm{C} .{ }^{1} \mathrm{H}$ NMR (200 MHz, DMSO-d $) \delta 7.40-7.08$ (m, 3H), $6.49\left(2, \mathrm{NH}_{2}\right), 4.83(\mathrm{~s}, 1 \mathrm{H}), 2.58(\mathrm{~d}, J 17.0 \mathrm{~Hz}, 1 \mathrm{H}), 2.50(\mathrm{~d}, J 17.0 \mathrm{~Hz}, 1 \mathrm{H}), 2.22(\mathrm{~s}, 2 \mathrm{H}), 1.66(\mathrm{~s}$, $3 \mathrm{H}), 1.02$ (s, 3H), 0.99 (s, 3H) ppm; ${ }^{13} \mathrm{C}$ NMR (100 MHz, DMSO-d $\left.d_{6}\right) \delta 196.11,164.93,159.91$, $151.75,136.04,129.99$, 128.44, 118.21, 115.94, 110.60, 109.30, 95.79, 50.84, 32.61, 32.09, 29.63, 29.32, 26.62, 10.23. MS (EI, $70 \mathrm{eV}): \mathrm{m} / z(\%) 404(8.5)\left[\mathrm{M}^{+}\right], 306(44.5), 227$ (99.9), 175 
(22.3), 142 (24.5), 128 (55.3), 98 (18.5). Anal. Calcd. for $\mathrm{C}_{19} \mathrm{H}_{19} \mathrm{BrN}_{2} \mathrm{O}_{3}: \mathrm{C}$ 56.59, $\mathrm{H} 4.75, \mathrm{~N}$ 6.95. Found: C 56.36, H 4.44, N 6.83\%.

9-(5-Amino-3-methylisoxazol-4-yl)-5-methoxy-3,3-dimethyl-2,3,4,9-tetrahydro-1H-xanthen1-one (11i). Colorless solid; mp 210-211 ${ }^{\circ} \mathrm{C} .{ }^{1} \mathrm{H}$ NMR (200 MHz, DMSO-d $\left.{ }_{6}\right) \delta$ 7.03-6.60 (m, $3 \mathrm{H}), 6.37\left(2, \mathrm{NH}_{2}\right), 4.76(\mathrm{~s}, 1 \mathrm{H}), 3.80(\mathrm{~s}, 3 \mathrm{H}), 2.59$ (d, J $\left.16.9 \mathrm{~Hz}, 1 \mathrm{H}\right), 2.52$ (d, J 16.9 Hz, 1H), $2.21(\mathrm{~s}, 2 \mathrm{H}), 1.62(\mathrm{~s}, 3 \mathrm{H}), 1.03(\mathrm{~s}, 3 \mathrm{H}), 0.99(\mathrm{~s}, 3 \mathrm{H}) \mathrm{ppm} ;{ }^{13} \mathrm{C}$ NMR (100 MHz, DMSO- $\left.d_{6}\right) \delta$ $197.00,166.87,164.69,159.18,147.93,125.42,124.58,121.22,111.67,110.85,96.02,56.71$, 51.07, 32.30, 28.92, 27.57, 25.75, 25.06, 10.42. MS (EI, $70 \mathrm{eV}): \mathrm{m} / z(\%) 355(2.6)\left[\mathrm{M}^{+}\right], 259$ (99.9), 233 (61.3), 180 (57.7), 159 (28.5), 123 (44.5), 98 (17.7). Anal. Calcd. for $\mathrm{C}_{20} \mathrm{H}_{22} \mathrm{~N}_{2} \mathrm{O}_{4}$ : C 67.78, H 6.26, N 7.90. Found: C 67.55, H 6.01, N 7.81\%.

9-(5-Amino-3-methylisoxazol-4-yl)-3,3-dimethyl-7-nitro-2,3,4,9-tetrahydro-1H-xanthen-1one (11j). Colorless solid; mp 241-242 ${ }^{\circ} \mathrm{C} .{ }^{1} \mathrm{H}$ NMR (200 MHz, DMSO- $\left.d_{6}\right) \delta 7.91-7.33$ (m, 3H), $6.68\left(2, \mathrm{NH}_{2}\right), 4.97(\mathrm{~s}, 1 \mathrm{H}), 2.58(\mathrm{~d}, J 16.9 \mathrm{~Hz}, 1 \mathrm{H}), 2.50(\mathrm{~d}, J 16.9 \mathrm{~Hz}, 1 \mathrm{H}), 2.22(\mathrm{~s}, 2 \mathrm{H}), 1.66$ (s, $3 \mathrm{H}), 1.02$ (s, 3H), 0.99 (s, 3H) ppm; ${ }^{13} \mathrm{C}$ NMR (100 MHz, DMSO-d $) \delta 189.90,170.87,165.69$, 159.18, 157.93, 143.42, 126.58, 122.22, 119.67, 109.85, 96.02, 51.71, 32.30, 30.92, 27.57, 26.75,

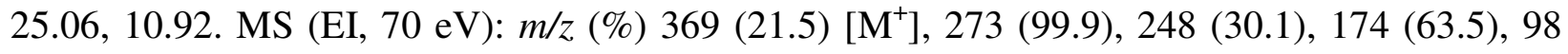
(85.7). Anal. Calcd. for $\mathrm{C}_{19} \mathrm{H}_{19} \mathrm{~N}_{3} \mathrm{O}_{5}$ : C 61.78, H 5.18, N 11.38. Found: C 61.60, H 4.86, N $11.30 \%$.

9-(5-Amino-3-methylisoxazol-4-yl)-3-(4-chlorophenyl)-2,3,4,9-tetrahydro-1H-xanthen-1one (11k). Colorless solid; ${ }^{1} \mathrm{H}$ NMR (200 MHz, DMSO- $\left.d_{6}\right) \delta 7.48-7.09(\mathrm{~m}, 8 \mathrm{H}), 6.40\left(2, \mathrm{NH}_{2}\right)$, $4.80(4.79)(\mathrm{s}, 1 \mathrm{H}), 3.46(\mathrm{~m}, 1 \mathrm{H}), 2.98(2.94)(\mathrm{m}, 2 \mathrm{H}), 2.75(2.68)(\mathrm{m}, 2 \mathrm{H}), 2.28(2.21)(\mathrm{s}, 3 \mathrm{H})$ ppm; ${ }^{13} \mathrm{C}$ NMR (100 MHz, DMSO- $\left.d_{6}\right) \delta 194.76,170.82,169.90,150.77,142.10,132.87,132.45$, $131.55,130.52,128.86,127.85,120.87,117.85,110.69,98.02,45.88,38.59,35.55,26.53,10.98$.

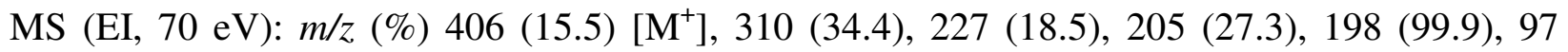
(25.3), 77 (16.1). Anal. Calcd. for $\mathrm{C}_{23} \mathrm{H}_{19} \mathrm{ClN}_{2} \mathrm{O}_{3}$ : C 67.90, H 4.71, N 6.89. Found: C 67.72, H 4.39, N $6.81 \%$.

9-(5-Amino-3-methylisoxazol-4-yl)-7-chloro-3-(4-chlorophenyl)-2,3,4,9-tetrahydro-1Hxanthen-1-one (11I). Colorless solid; ${ }^{1} \mathrm{H}$ NMR (200 MHz, DMSO- $\left.d_{6}\right) \delta$ 7.47-6.79 (m, 7H), 6.47 $\left(2, \mathrm{NH}_{2}\right), 4.83(4.82)(\mathrm{s}, 1 \mathrm{H}), 3.52(\mathrm{~m}, 1 \mathrm{H}), 3.08(3.04)(\mathrm{m}, 2 \mathrm{H}), 2.66(2.63)(\mathrm{m}, 2 \mathrm{H}), 2.20(2.19)(\mathrm{s}$, $3 \mathrm{H}) \mathrm{ppm} ;{ }^{13} \mathrm{C}$ NMR $\left(100 \mathrm{MHz}, \mathrm{DMSO}-d_{6}\right) \delta 194.76,171.82,169.90,151.77,142.10,133.87$, $132.45,131.55,130.52,128.86,127.75,121.87,116.85,110.89,98.12,45.98,38.69,34.55$, 27.85, 11.08. MS (EI, $70 \mathrm{eV}): m / z$ (\%) $440(4.3)\left[\mathrm{M}^{+}\right], 345$ (33.5), 262 (18.9), 245 (99.9), 205 (17.7), 97 (57.3), 77 (39.1). Anal. Calcd. for $\mathrm{C}_{23} \mathrm{H}_{18} \mathrm{Cl}_{2} \mathrm{~N}_{2} \mathrm{O}_{3}: \mathrm{C}$ 62.60, $\mathrm{H}$ 4.11, $\mathrm{N}$ 6.35. Found: $\mathrm{C}$ $62.37, \mathrm{H} 3.79$, N 6.25\%.

9-(5-Amino-3-methylisoxazol-4-yl)-7-bromo-3-(4-chlorophenyl)-2,3,4,9-tetrahydro-1Hxanthen-1-one (11m). Colorless solid; ${ }^{1} \mathrm{H}$ NMR (200 MHz, DMSO-d $d_{6} \delta$ 7.57-7.09 (m, 7H), $6.48\left(2, \mathrm{NH}_{2}\right), 4.80(4.79)(\mathrm{s}, 1 \mathrm{H}), 3.50(\mathrm{~m}, 1 \mathrm{H}), 3.08(3.04)(\mathrm{m}, 2 \mathrm{H}), 2.66(2.63)(\mathrm{m}, 2 \mathrm{H})$, $2.20(2.19)(\mathrm{s}, 3 \mathrm{H}) \mathrm{ppm} ;{ }^{13} \mathrm{C} \mathrm{NMR}\left(100 \mathrm{MHz}, \mathrm{DMSO}-d_{6}\right) \delta 195.86,171.82,169.10,152.16$, $143.29,134.85,132.15,131.05,130.52,128.84,127.15,121.07,116.15,111.89,97.10,45.98$, 36.69, 33.55, 28.85, 10.98. MS (EI, $70 \mathrm{eV}): m / z(\%) 486(7.5)\left[\mathrm{M}^{+}\right], 389$ (73.4), 304 (19.5), 277 
(67.4), 197 (99.9), 111 (23.1), 92 (16.3), 77 (39.1). Anal. Calcd. for $\mathrm{C}_{23} \mathrm{H}_{18} \mathrm{BrClN}_{2} \mathrm{O}_{3}$ : C 56.87, $\mathrm{H}$ 3.74, N 5.77. Found: C 56.69, H 3.43, N 5.67\%.

9-(5-Amino-3-methylisoxazol-4-yl)-3-(4-chlorophenyl)-5-methoxy-2,3,4,9-tetrahydro-1Hxanthen-1-one (11n). Colorless solid; ${ }^{1} \mathrm{H}$ NMR (200 MHz, DMSO- $\left.d_{6}\right) \delta 7.57-7.09(\mathrm{~m}, 7 \mathrm{H}), 6.48$ $\left(2, \mathrm{NH}_{2}\right), 4.80(4.79)(\mathrm{s}, 1 \mathrm{H}), 3.80(3.78)(\mathrm{s}, 3 \mathrm{H}), 3.46(\mathrm{~m}, 1 \mathrm{H}), 2.99(2.94)(\mathrm{m}, 2 \mathrm{H}), 2.66(2.60)$ $(\mathrm{m}, 2 \mathrm{H}), 2.24(2.22)(\mathrm{s}, 3 \mathrm{H}) \mathrm{ppm} ;{ }^{13} \mathrm{C} \mathrm{NMR}\left(100 \mathrm{MHz}, \mathrm{DMSO}-d_{6}\right) \delta 194.86,172.89,169.95$, $153.16,144.29,136.85,134.15,132.05,131.52,128.90,126.15,121.97,115.15,111.09,98.10$, 54.34, 45.98, 36.69, 33.55, 28.85, 11.08. MS (EI, $70 \mathrm{eV}): \mathrm{m} / z(\%) 437$ (3.8) $\left[\mathrm{M}^{+}\right], 341$ (63.5), 233 (12.5), 229 (17.2), 228 (99.9), 93 (18.3), 77 (12.3). Anal. Calcd. for $\mathrm{C}_{24} \mathrm{H}_{21} \mathrm{ClN}_{2} \mathrm{O}_{4}$ : C 65.98, H 4.84, N 6.41. Found: C 65.77, H 4.55, N 6.32\%.

9-(5-Amino-3-methylisoxazol-4-yl)-3-(4-chlorophenyl)-7-nitro-2,3,4,9-tetrahydro-1Hxanthen-1-one (110). Colorless solid; ${ }^{1} \mathrm{H}$ NMR $\left(200 \mathrm{MHz}, \mathrm{DMSO}-d_{6}\right) \delta 7.88-7.09(\mathrm{~m}, 7 \mathrm{H}), 6.65$ $\left(2, \mathrm{NH}_{2}\right), 4.98(4.97)(\mathrm{s}, 1 \mathrm{H}), 3.51(\mathrm{~m}, 1 \mathrm{H}), 3.05(2.95)(\mathrm{m}, 2 \mathrm{H}), 2.68(2.63)(\mathrm{m}, 2 \mathrm{H}), 2.32(2.30)(\mathrm{s}$, $3 \mathrm{H}) \mathrm{ppm} ;{ }^{13} \mathrm{C}$ NMR $\left(100 \mathrm{MHz}, \mathrm{DMSO}-d_{6}\right) \delta 195.86,171.80,165.12,151.22,141.10,134.17$, $132.16,131.22$, 130.10, 128.17, 126.15, 122.07, 119.15, 111.89, 97.10, 45.98, 36.69, 33.55,

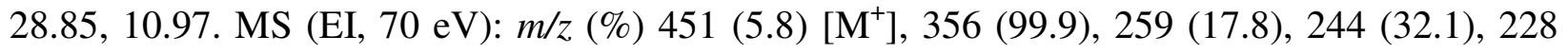
(16.5), 97 (14.5). Anal. Calcd. For $\mathrm{C}_{23} \mathrm{H}_{18} \mathrm{ClN}_{3} \mathrm{O}_{5}$ : C 61.14, H 4.02, N 9.30. Found: C 60.91, H $3.75, \mathrm{~N} 9.17 \%$.

General procedure for synthesis of 12a-o. A mixture of 5-amino-3-methylisoxazole 1 (1 mmol), aldehydes 9a-e $(2 \mathrm{mmol})$ and cyclic $\beta$-di-ketones 3a-c $(1 \mathrm{mmol})$ in DMF $(2 \mathrm{~mL})$ was heated to reflux for $5 \mathrm{~min}$ in a roundbottom flask equipped with a condenser. Then after cooling ethanol $(10 \mathrm{~mL})$ was added and the precipitate formed was filtered out to give the solid compounds, which were washed with ethanol and air dried.

9-(5-\{[(2-Hydroxyphenyl)methylene]amino\}-3-methylisoxazol-4-yl)-2,3,4,9-tetrahydro-1Hxanthen-1-one (12a). Colorless solid; mp 263-264 ${ }^{\circ} \mathrm{C} .{ }^{1} \mathrm{H}$ NMR $\left(200 \mathrm{MHz}, \mathrm{DMSO}-d_{6}\right) \delta 11.28$ (s, 1OH), $9.00(\mathrm{~s}, 1 \mathrm{H}), 7.80-6.97(\mathrm{~m}, 8 \mathrm{H}), 4.97(\mathrm{~s}, 1 \mathrm{H}), 3.48(\mathrm{~m}, 1 \mathrm{H}), 2.29(\mathrm{~m}, 2 \mathrm{H}), 2.24(\mathrm{~s}, 3 \mathrm{H})$, $1.94(\mathrm{~m}, 2 \mathrm{H}) \mathrm{ppm} ;{ }^{13} \mathrm{C}$ NMR $\left(100 \mathrm{MHz}, \mathrm{DMSO}-d_{6}\right) \delta 194.45,163.08,160.23,159.20,149.65$, $135.43,131.82$, 130.40, 128.61, 125.76, 123.46, 120.79, 120.26, 117.52, 116.94, 114.93, 110.76,

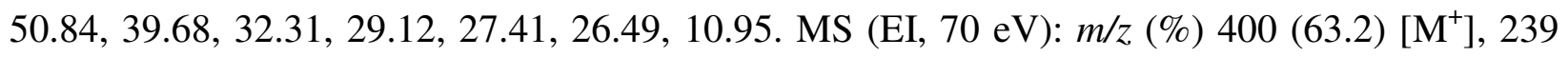
(55.4), 199 (87.1), 181 (32.3), 152 (20.3), 127 (47.9), 115 (71.6), 102 (59.6), 93 (26.2), 77 (99.9), 65 (96.1), 42 (27.5). Anal. Calcd. for $\mathrm{C}_{24} \mathrm{H}_{20} \mathrm{~N}_{2} \mathrm{O}_{4}$ : C 71.99, H 5.03, N 7.00. Found: C 71.81, H $4.75, \mathrm{~N} 6.93 \%$.

7-Chloro-9-(5-\{[(5-chloro-2-hydroxyphenyl)methylene $]$ amino $\}-3-m e t h y l i s o x a z o l-4-y l)-$ 2,3,4,9-tetrahydro-1H-xanthen-1-one (12b). Colorless solid; mp 232-233 ${ }^{\circ} \mathrm{C} .{ }^{1} \mathrm{H}$ NMR (200 MHz, DMSO- $\left.d_{6}\right) \delta 11.16(\mathrm{~s}, 1 \mathrm{OH}), 8.86(\mathrm{~s}, 1 \mathrm{H}), 7.83-7.00(\mathrm{~m}, 6 \mathrm{H}), 4.91(\mathrm{~s}, 1 \mathrm{H}), 2.68(\mathrm{~m}, 2 \mathrm{H})$, $2.38(\mathrm{~s}, 3 \mathrm{H}), 2.28(\mathrm{~m}, 2 \mathrm{H}), 1.94(\mathrm{~m}, 2 \mathrm{H}) \mathrm{ppm} ;{ }^{13} \mathrm{C}$ NMR (100 MHz, DMSO-d $) \delta 196.05$, $164.78,161.33,160.20,149.05,138.43,135.82,133.40,128.61,125.76,123.46,120.79,120.26$, $117.52,116.94,114.93,108.76,51.84,39.08,32.30,29.12,28.41,23.49,11.95$. MS (EI, 70 eV): 
$\mathrm{m} / z(\%) 468$ (3.2) $\left[\mathrm{M}^{+}\right], 327$ (23.3), 237 (28.5), 233 (99.9), 139 (17.7), 96 (17.4). Anal. Calcd. for $\mathrm{C}_{24} \mathrm{H}_{18} \mathrm{Cl}_{2} \mathrm{~N}_{2} \mathrm{O}_{4}$ : C 61.42, H 3.87, N 5.97. Found: C 61.20, H 3.55, N 5.89\%.

7-Bromo-9-(5-\{[(5-bromo-2-hydroxyphenyl)methylene]amino\}-3-methylisoxazol-4-yl)2,3,4,9-tetrahydro-1H-xanthen-1-one (12c). Colorless solid; mp 248-249 ${ }^{\circ} \mathrm{C} .{ }^{1} \mathrm{H}$ NMR (200 MHz, DMSO- $\left.d_{6}\right) \delta 11.10(\mathrm{~s}, 1 \mathrm{OH}), 8.82(\mathrm{~s}, 1 \mathrm{H}), 7.75-6.98(\mathrm{~m}, 6 \mathrm{H}), 4.90(\mathrm{~s}, 1 \mathrm{H}), 2.68(\mathrm{~m}, 2 \mathrm{H})$, $2.38(\mathrm{~s}, 3 \mathrm{H}), 2.28(\mathrm{~m}, 2 \mathrm{H}), 1.94(\mathrm{~m}, 2 \mathrm{H}) \mathrm{ppm} ;{ }^{13} \mathrm{C}$ NMR (100 MHz, DMSO-d 6$) \delta 195.65$, $165.18,161.03,160.25,150.65,133.43,131.82,130.60,128.61,125.96,123.86,120.09,119.26$, $117.52,116.95,114.03,110.16,51.84,40.68,33.31,29.22,27.81,26.09,10.55$. MS (EI, $70 \mathrm{eV})$ : $\mathrm{m} / \mathrm{z}(\%) 557$ (4.5) $\left[\mathrm{M}^{+}\right], 372$ (51.1), 278 (99.9), 185 (41.2), 170 (14.2), 78 (14.5). Anal. Calcd. for $\mathrm{C}_{24} \mathrm{H}_{18} \mathrm{Br}_{2} \mathrm{~N}_{2} \mathrm{O}_{4}$ : C 51.64, H 3.25, N 5.02. Found: C 51.41, H 3.01, N 4.91\%.

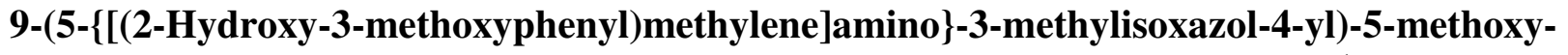
2,3,4,9-tetrahydro-1H-xanthen-1-one (12d). Colorless solid; mp 211-212 ${ }^{\circ} \mathrm{C} .{ }^{1} \mathrm{H}$ NMR $(200$ $\left.\mathrm{MHz}, \mathrm{DMSO}-d_{6}\right) \delta 11.04(\mathrm{~s}, 1 \mathrm{OH}), 8.67(\mathrm{~s}, 1 \mathrm{H}), 7.38-6.49(\mathrm{~m}, 6 \mathrm{H}), 4.95(\mathrm{~s}, 1 \mathrm{H}), 3.73(\mathrm{~s}, 3 \mathrm{H})$, $2.68(\mathrm{~m}, 2 \mathrm{H}), 2.20(\mathrm{~s}, 3 \mathrm{H}), 2.28(\mathrm{~m}, 2 \mathrm{H}), 1.94(\mathrm{~m}, 2 \mathrm{H}) \mathrm{ppm} ;{ }^{13} \mathrm{C}$ NMR $\left(100 \mathrm{MHz}, \mathrm{DMSO}-d_{6}\right) \delta$ $194.65,167.18,165.23,160.20,149.65,135.43,131.82,130.40,128.61,125.76,123.46,120.79$, 120.26, 117.52, 116.94, 114.93, 110.76, 56.35, 55.86, 50.84, 39.68, 32.31, 29.92, 28.41, 26.89, 10.90. MS (EI, $70 \mathrm{eV}): \mathrm{m} / z$ (\%) 460 (11.5) $\left[\mathrm{M}^{+}\right], 232$ (24.3), 230 (99.9), 182 (37.1), 123 (14.1). Anal. Calcd. for $\mathrm{C}_{26} \mathrm{H}_{24} \mathrm{~N}_{2} \mathrm{O}_{6}$ : C 67.82, H 5.25, N 6.08. Found: C 67.64, H 5.00, N 5.95\%.

9-(5-\{[(2-Hydroxy-5-nitrophenyl)methylene]amino\}-3-methylisoxazol-4-yl)-7-nitro-2,3,4,9tetrahydro-1H-xanthen-1-one (12e). Colorless solid; mp 195-196 ${ }^{\circ} \mathrm{C} .{ }^{1} \mathrm{H}$ NMR $(200 \mathrm{MHz}$, DMSO-d $\left.d_{6}\right) \delta 11.10(\mathrm{~s}, 1 \mathrm{OH}), 8.97(\mathrm{~s}, 1 \mathrm{H}), 8.38-6.89(\mathrm{~m}, 6 \mathrm{H}), 4.98(\mathrm{~s}, 1 \mathrm{H}), 2.68(\mathrm{~m}, 2 \mathrm{H}), 2.20(\mathrm{~s}$, $3 \mathrm{H}), 2.28(\mathrm{~m}, 2 \mathrm{H}), 2.04(\mathrm{~m}, 2 \mathrm{H}) \mathrm{ppm} ;{ }^{13} \mathrm{C}$ NMR $\left(100 \mathrm{MHz}, \mathrm{DMSO}-d_{6}\right) \delta 195.15,170.28$, $168.23,164.20,155.65,145.43,135.82,130.40,129.61,125.96,123.46,120.79,120.26,117.52$, 116.94, 114.93, 109.76, 48.94, 38.68, 32.31, 30.92, 29.41, 27.89, 11.10. MS (EI, $70 \mathrm{eV}): \mathrm{m} / z(\%)$ 490 (7.4) $\left[\mathrm{M}^{+}\right], 245$ (99.9), 182 (36.6), 175 (14.3), 139 (24.2), 95 (18.9), 77 (44.2). Anal. Calcd. for $\mathrm{C}_{24} \mathrm{H}_{18} \mathrm{~N}_{4} \mathrm{O}_{8}$ : C 58.78, H 3.70, N 11.42. Found: C 58.60, H 3.41, N 11.33\%.

9-(5-\{[(2-Hydroxyphenyl)methylene]amino\}-3-methylisoxazol-4-yl)-3,3-dimethyl-2,3,4,9tetrahydro-1H-xanthen-1-one (12f). Colorless solid; mp 180-181 ${ }^{\circ} \mathrm{C} .{ }^{1} \mathrm{H}$ NMR (200 MHz, DMSO-d $)_{6} \delta 11.10(\mathrm{~s}, 1 \mathrm{OH}), 8.96(\mathrm{~s}, 1 \mathrm{H}), 7.80-6.96(\mathrm{~m}, 8 \mathrm{H}), 4.90(\mathrm{~s}, 1 \mathrm{H}), 2.64$ (d, J 16.9 Hz, $1 \mathrm{H}), 2.40(\mathrm{~d}, J 16.9 \mathrm{~Hz}, 1 \mathrm{H}), 2.30(\mathrm{~d}, J 16.1 \mathrm{~Hz}, 1 \mathrm{H}), 2.10(\mathrm{~d}, J 17.0 \mathrm{~Hz}, 1 \mathrm{H}), 2.25(\mathrm{~s}, 3 \mathrm{H}), 1.02$ $(\mathrm{s}, 3 \mathrm{H}), 0.83$ (s, 3H) ppm; ${ }^{13} \mathrm{C}$ NMR (100 MHz, DMSO-d $) \delta 196.65,165.18,161.23,160.20$, $149.65,135.43,131.82$, 130.40, 128.61, 125.76, 123.46, 120.79, 120.26, 117.52, 116.94, 114.93, 110.76, 50.84, 39.68, 32.31, 29.12, 27.41, 26.49, 10.95. MS (EI, $70 \mathrm{eV}): \mathrm{m} / \mathrm{z}(\%) 428(3.5)\left[\mathrm{M}^{+}\right]$, 322 (19.6), 228 (99.9), 202 (37.5), 123 (45.6), 95 (18.5). Anal. Calcd. for $\mathrm{C}_{26} \mathrm{H}_{24} \mathrm{~N}_{2} \mathrm{O}_{4}: \mathrm{C} 72.88$, H 5.65, N 6.54. Found: C 72.70, H 5.36, N 6.44\%.

7-Chloro-9-(5-\{[(5-chloro-2-hydroxyphenyl)methylene]amino\}-3-methylisoxazol-4-yl)-3,3dimethyl-2,3,4,9-tetrahydro-1H-xanthen-1-one (12g). Colorless solid; mp 241-242 ${ }^{\circ} \mathrm{C}$. ${ }^{1} \mathrm{H}$ NMR (200 MHz, DMSO-d $) \delta 10.68(\mathrm{~s}, 1 \mathrm{OH}), 8.80(\mathrm{~s}, 1 \mathrm{H}), 7.34-6.69(\mathrm{~m}, 6 \mathrm{H}), 5.01(\mathrm{~s}, 1 \mathrm{H})$, $2.64(\mathrm{~d}, J 17.0 \mathrm{~Hz}, 1 \mathrm{H}), 2.40(\mathrm{~d}, J 17.0 \mathrm{~Hz}, 1 \mathrm{H}), 2.30$ (d, $J 16.1 \mathrm{~Hz}, 1 \mathrm{H}), 2.10$ (d, $J 16.1 \mathrm{~Hz}, 1 \mathrm{H})$, 2.21 (s, 3H), 1.02 (s, 3H), 0.83 (s, 3H) ppm; ${ }^{13} \mathrm{C}$ NMR (100 MHz, DMSO-d $) \delta 193.45,170.18$, 
$168.45,156.21,150.65,135.58,131.95,130.10,128.56,124.96,123.90,122.15,120.28,118.14$, 117.84, 115.25, 106.12, 50.04, 39.85, 32.49, 30.92, 28.08, 27.10, 11.02. MS (EI, $70 \mathrm{eV}): \mathrm{m} / \mathrm{z}(\%)$ 496 (11.2) $\left[\mathrm{M}^{+}\right], 263$ (99.9), 235 (30.1), 165 (28.3), 129 (43.5), 122(16.7). Anal. Calcd. for $\mathrm{C}_{26} \mathrm{H}_{22} \mathrm{Cl}_{2} \mathrm{~N}_{2} \mathrm{O}_{4}$ : C 62.79, H 4.46, N 5.63. Found: C 62.61, H 4.18, N 5.55\%.

7-Bromo-9-(5-[[(5-bromo-2-hydroxyphenyl)methylene]amino]-3-methylisoxazol-4-yl)-3,3dimethyl-2,3,4,9-tetrahydro-1 $\boldsymbol{H}$-xanthen-1-one (12h). Colorless solid; mp 256-257 ${ }^{\circ} \mathrm{C} .{ }^{1} \mathrm{H}$ NMR $\left(200 \mathrm{MHz}\right.$, DMSO- $\left.d_{6}\right) \delta 11.07(\mathrm{~s}, 1 \mathrm{OH}), 8.82(\mathrm{~s}, 1 \mathrm{H}), 7.80-7.01(\mathrm{~m}, 6 \mathrm{H}), 4.92(\mathrm{~s}, 1 \mathrm{H})$, $2.58(\mathrm{~d}, J 17.0 \mathrm{~Hz}, 1 \mathrm{H}), 2.39(\mathrm{~d}, J 17.0 \mathrm{~Hz}, 1 \mathrm{H}), 2.32(\mathrm{~d}, J 16.1 \mathrm{~Hz}, 1 \mathrm{H}), 2.11(\mathrm{~d}, J 16.1 \mathrm{~Hz}, 1 \mathrm{H})$, $2.30(\mathrm{~s}, 3 \mathrm{H}), 1.02(\mathrm{~s}, 3 \mathrm{H}), 0.88$ (s, 3H) ppm; ${ }^{13} \mathrm{C}$ NMR (100 MHz, DMSO- $\left.d_{6}\right) \delta 190.45,173.08$, $168.25,155.21,150.65,135.48,130.95,130.01,127.56,125.86,123.40,122.10,120.23,118.14$, $117.84,111.55,108.01,50.84,39.90,32.39,30.82,27.08,26.09,11.08$. MS (EI, $70 \mathrm{eV}): \mathrm{m} / \mathrm{z}(\%)$ 586 (13.4) $\left[\mathrm{M}^{+}\right], 402$ (34.4), 307 (99.9), 281 (54.1), 171 (17.8), 123 (34.1). Anal. Calcd. for $\mathrm{C}_{26} \mathrm{H}_{22} \mathrm{Br}_{2} \mathrm{~N}_{2} \mathrm{O}_{4}$ : C 53.27, H 3.78, N 4.78. Found: C 53.10, H 3.49, N 4.67\%.

9-(5-\{[(2-Hydroxy-3-methoxyphenyl)methylene]amino\}-3-methylisoxazol-4-yl)-5-methoxy3,3-dimethyl-2,3,4,9-tetrahydro-1 $\boldsymbol{H}$-xanthen-1-one (12i). Colorless solid; mp 211-212 ${ }^{\circ} \mathrm{C} .{ }^{1} \mathrm{H}$ NMR $\left(200 \mathrm{MHz}\right.$, DMSO- $\left.d_{6}\right) \delta 10.77(\mathrm{~s}, 1 \mathrm{OH}), 8.98(\mathrm{~s}, 1 \mathrm{H}), 7.42-6.62(\mathrm{~m}, 6 \mathrm{H}), 4.90(\mathrm{~s}, 1 \mathrm{H})$, $3.81(\mathrm{~s}, 6 \mathrm{H}), 2.64(\mathrm{~d}, J 16.9 \mathrm{~Hz}, 1 \mathrm{H}), 2.40(\mathrm{~d}, J 16.9 \mathrm{~Hz}, 1 \mathrm{H}), 2.30(\mathrm{~d}, J 16.0 \mathrm{~Hz}, 1 \mathrm{H}), 2.10(\mathrm{~d}, J$ $16.0 \mathrm{~Hz}, 1 \mathrm{H}), 2.26(\mathrm{~s}, 3 \mathrm{H}), 1.02(\mathrm{~s}, 3 \mathrm{H}), 0.84(\mathrm{~s}, 3 \mathrm{H}) \mathrm{ppm} ;{ }^{13} \mathrm{C}$ NMR $\left(100 \mathrm{MHz}\right.$, DMSO- $\left.d_{6}\right) \delta$ 194.65, 164.90, 160.25, 158.21, 150.65, 145.48, 130.81, 130.08, 128.69, 125.06, 124.41, 122.80, $120.86,118.40,117.04,115.93,110.01,55.64,54.35,50.04,39.88,32.35,30.12,28.48,27.49$,

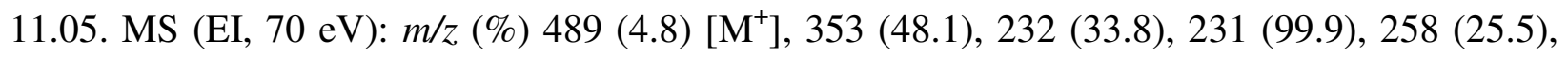
124 (48.9), 77 (16.9). Anal. Calcd. for $\mathrm{C}_{28} \mathrm{H}_{28} \mathrm{~N}_{2} \mathrm{O}_{6}$ : C 68.84, H 5.78, N 5.73. Found: C 68.67, H $5.50, \mathrm{~N} 5.64 \%$.

9-(5-\{[(2-Hydroxy-5-nitrophenyl)methylene]amino\}-3-methylisoxazol-4-yl)-3,3-dimethyl-7nitro-2,3,4,9-tetrahydro-1H-xanthen-1-one (12j). Colorless solid; mp 214-215 ${ }^{\circ} \mathrm{C} .{ }^{1} \mathrm{H}$ NMR $\left(200 \mathrm{MHz}\right.$, DMSO- $\left.d_{6}\right) \delta 10.98(\mathrm{~s}, 1 \mathrm{OH}), 8.82(\mathrm{~s}, 1 \mathrm{H}), 7.84-7.05(\mathrm{~m}, 6 \mathrm{H}), 4.99(\mathrm{~s}, 1 \mathrm{H}), 2.62(\mathrm{~d}, J$ $17.0 \mathrm{~Hz}, 1 \mathrm{H}), 2.40(\mathrm{~d}, J 17.0 \mathrm{~Hz}, 1 \mathrm{H}), 2.28(\mathrm{~d}, J 16.1 \mathrm{~Hz}, 1 \mathrm{H}), 2.10(\mathrm{~d}, J 17.0 \mathrm{~Hz}, 1 \mathrm{H}), 2.22(\mathrm{~s}$, $3 \mathrm{H}), 1.01$ (s, 3H), 0.89 (s, 3H) ppm; ${ }^{13} \mathrm{C}$ NMR (100 MHz, DMSO- $\left.d_{6}\right) \delta 188.45,170.18,168.45$, 158.21, 152.65, 145.58, 135.95, 128.10, 126.56, 124.96, 122.90, 122.15, 120.28, 118.14, 117.84, 111.25, 104.12, 51.04, 40.05, 37.49, 34.92, 28.08, 27.10, 11.01. MS (EI, $70 \mathrm{eV}): \mathrm{m} / \mathrm{z}$ (\%) 518 (4.5) $\left[\mathrm{M}^{+}\right], 368$ (99.9), 272 (28.3), 247 (43.5), 139 (56.8), 122 (16.6), 95 (9.8). Anal. Calcd. for $\mathrm{C}_{26} \mathrm{H}_{22} \mathrm{~N}_{4} \mathrm{O}_{8}$ : C 60.23, H 4.28, N 10.81. Found: C 60.01, H 4.00, N $10.70 \%$.

3-(4-Chlorophenyl)-9-(5-\{[(2-hydroxyphenyl)methylene]amino\}-3-methylisoxazol-4-yl)2,3,4,9-tetrahydro-1 $\boldsymbol{H}$-xanthen-1-one (12k). Colorless solid; ${ }^{1} \mathrm{H}$ NMR $\left(200 \mathrm{MHz}\right.$, DMSO- $\left.d_{6}\right) \delta$ 11.35(11.18) (s, 1OH), 9.03(8.95), (s, 1H), 7.80-6.97 (m, 12H), 5.02(4.97) (s, 1H), $3.52(\mathrm{~m}, 1 \mathrm{H})$, 2.98(2.94) (m, 2H), 2.75(2.68) (m, 2H), 2.28(2.21) (s, 3H) ppm; ${ }^{13} \mathrm{C}$ NMR (100 MHz, DMSO$\left.d_{6}\right) \delta 195.84,166.29,165.32,164.40,162.14,160.48,149.31,142.19,135.35,131.30,130.70$, $129.33,125.82,123.15,120.60,117.36,116.82,111.07,43.88,37.59,34.55,26.33,10.98$. MS (EI, $70 \mathrm{eV}): m / z(\%) 510(14.8)\left[\mathrm{M}^{+}\right], 400$ (10.1), 294 (29.5), 205 (19.8), 199 (99.9), 113 (14.9), 
93 (27.5). Anal. Calcd. for $\mathrm{C}_{30} \mathrm{H}_{23} \mathrm{ClN}_{2} \mathrm{O}_{4}$ : C 70.52, H 4.54, N 5.48. Found: C 70.34, H 4.23, N $5.40 \%$.

7-Chloro-9-(5-\{[(5-chloro-2-hydroxyphenyl)methylene]amino\}-3-methylisoxazol-4-yl)-3-(4chlorophenyl)-2,3,4,9-tetrahydro-1H-xanthen-1-one (12l). Colorless solid; ${ }^{1} \mathrm{H}$ NMR (200 MHz, DMSO- $\left.d_{6}\right) \delta 11.09(\mathrm{~s}, 1 \mathrm{OH}), 8.89(8.75)(\mathrm{s}, 1 \mathrm{H}), 7.83-6.93(\mathrm{~m}, 10 \mathrm{H}), 4.98(4.91)(\mathrm{s}, 1 \mathrm{H})$, $3.53(\mathrm{~m}, 1 \mathrm{H}), 3.08(3.04)(\mathrm{m}, 2 \mathrm{H}), 2.66(2.63)(\mathrm{m}, 2 \mathrm{H}), 2.40(2.39)(\mathrm{s}, 3 \mathrm{H}) \mathrm{ppm} ;{ }^{13} \mathrm{C}$ NMR $(100$ MHz, DMSO- $\left.d_{6}\right) \delta 195.67,165.72,161.10,158.29,148.27,142.08,134.49,131.68,129.74$, $128.91,125.69,123.89,122.82,119.34,118.47,115.55,111.34,43.90,37.56,34.50,26.32$,

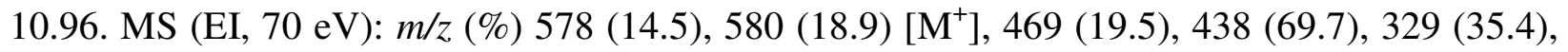
237 (16.6), 234 (99.9), 204 (17.2), 129 (34.5), 95 (14.2). Anal. Calcd. for $\mathrm{C}_{30} \mathrm{H}_{21} \mathrm{Cl}_{3} \mathrm{~N}_{2} \mathrm{O}_{4}$ : C 62.14, H 3.65, N 4.83. Found: C 61.91, H 3.33, N 4.71\%.

7-Bromo-9-(5-\{[(5-bromo-2-hydroxyphenyl)methylene]amino\}-3-methylisoxazol-4-yl)-3-(4chlorophenyl)-2,3,4,9-tetrahydro-1H-xanthen-1-one (12m). Colorless solid; ${ }^{1} \mathrm{H}$ NMR (200 MHz, DMSO- $\left.d_{6}\right) \delta 11.09(\mathrm{~s}, 1 \mathrm{OH}), 8.87(8.72)(\mathrm{s}, 1 \mathrm{H}), 7.95-6.91(\mathrm{~m}, 10 \mathrm{H}), 4.97(4.89)(\mathrm{s}, 1 \mathrm{H})$, $3.52(\mathrm{~m}, 1 \mathrm{H}), 3.07(3.00)(\mathrm{m}, 2 \mathrm{H}), 2.67(2.60)(\mathrm{m}, 2 \mathrm{H}), 2.41(2.40)(\mathrm{s}, 3 \mathrm{H}) \mathrm{ppm} ;{ }^{13} \mathrm{C}$ NMR $(100$ $\left.\mathrm{MHz}, \mathrm{DMSO}-d_{6}\right) \delta 194.59,165.66,164.72,160.40,159.14,148.48,142.09,137.09,132.35$, $129.30,128.70,126.13,123.34,119.15,118.60,117.06,111.39,43.91,42.57,37.59,36.61$,

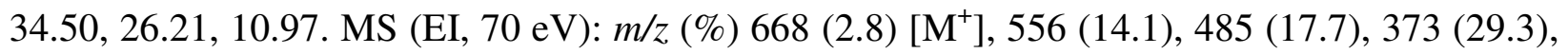
279 (29.1), 198 (99.9), 172 (19.5), 113 (14.5). Anal. Calcd. for $\mathrm{C}_{30} \mathrm{H}_{21} \mathrm{Br}_{2} \mathrm{ClN}_{2} \mathrm{O}_{4}$ : C 53.88, $\mathrm{H}$ 3.17, N 4.19. Found: C 53.70, H 2.85, N 4.10\%.

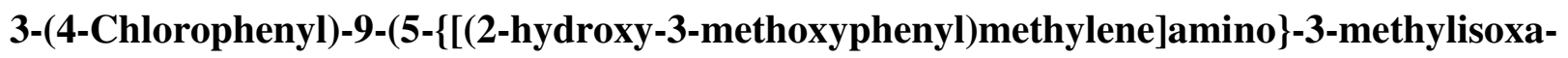
zol-4-yl)-5-methoxy-2,3,4,9-tetrahydro-1H-xanthen-1-one (12n). Colorless solid; ${ }^{1} \mathrm{H}$ NMR $\left(200 \mathrm{MHz}, \mathrm{DMSO}-d_{6}\right) \delta 10.91(\mathrm{~s}, 1 \mathrm{OH}), 9.04(8.99)(\mathrm{s}, 1 \mathrm{H}), 7.35-6.40(\mathrm{~m}, 10 \mathrm{H}), 5.00(4.94)(\mathrm{s}$, $1 \mathrm{H}), 3.81(3.74)(\mathrm{s}, 6 \mathrm{H}), 3.46(\mathrm{~m}, 1 \mathrm{H}), 2.96(2.88)(\mathrm{m}, 2 \mathrm{H}), 2.71(2.61)(\mathrm{m}, 2 \mathrm{H}), 2.24(2.13)(\mathrm{s}, 3 \mathrm{H})$ ppm; ${ }^{13} \mathrm{C}$ NMR (100 MHz, DMSO- $\left.d_{6}\right) \delta 194.67,163.72,162.10,156.29,149.27,141.08,135.49$, $131.68,129.84,128.91,124.69,123.89,122.80,118.34,116.47,115.55,109.34,55.47,53.98$,

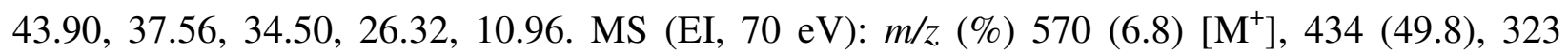
(17.4), 231 (14.3), 228 (99.9), 123 (74.1), 111 (19.4), 95 (32.1). Anal. Calcd. for $\mathrm{C}_{32} \mathrm{H}_{27} \mathrm{ClN}_{2} \mathrm{O}_{6}$ : C 67.31, H 4.77, N 4.91. Found: C 67.13, H 4.45, N 4.83\%.

3-(4-Chlorophenyl)-9-(5-\{[(2-hydroxy-5-nitrophenyl)methylene]amino\}-3-methylisoxazol-4yl)-7-nitro-2,3,4,9-tetrahydro-1H-xanthen-1-one (12o). Colorless solid; ${ }^{1} \mathrm{H}$ NMR (200 MHz, DMSO-d $\left.d_{6}\right) \delta 11.01(\mathrm{~s}, 1 \mathrm{OH}), 9.03(8.99)(\mathrm{s}, 1 \mathrm{H}), 7.48-6.40(\mathrm{~m}, 10 \mathrm{H}), 5.01(4.95)(\mathrm{s}, 1 \mathrm{H}), 3.49(\mathrm{~m}$, 1H), 2.99(2.90) (m, 2H), 2.79(2.68) (m, 2H), 2.34(2.23) (s, 3H) ppm; ${ }^{13} \mathrm{C}$ NMR (100 MHz, DMSO- $\left.d_{6}\right) \delta 191.67,165.72,162.30,155.29,150.27,141.08,132.49,131.68,129.84,127.91$, $124.69,123.89,122.80,115.34,111.47,110.55,105.34,42.90,37.56,34.50,25.32,11.06 . \mathrm{MS}$ (EI, $70 \mathrm{eV}): m / z(\%) 600(4.5)\left[\mathrm{M}^{+}\right], 490$ (18.5), 451 (34.1), 339 (74.1), 247 (17.1), 244 (99.9), 139 (71.1), 113 (14.4), 95 (21.7). Anal. Calcd. for $\mathrm{C}_{30} \mathrm{H}_{21} \mathrm{ClN}_{4} \mathrm{O}_{8}$ : C 59.96, H 3.52, N 9.32. Found: C 59.77, H 3.20, N 9.23\%. 


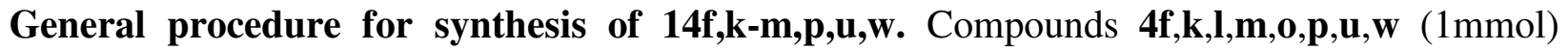
were dissolved in methanol at reflux, and then NBS $(1 \mathrm{mmol})$ was added. The mixture was heated for an $1 \mathrm{~h}$, then cooled to room temperature and the precipitate formed was filtered off.

3-Methyl-4-(4-nitrophenyl)-7,8-dihydroisoxazolo[5,4-b]quinolin-5(6H)-one (14f). Colorless solid, mp 179-180 ${ }^{\circ} \mathrm{C} .{ }^{1} \mathrm{H}$ NMR $\left(200 \mathrm{MHz}, \mathrm{DMSO}-d_{6}\right) \delta 8.30-7.58(\mathrm{~m}, 4 \mathrm{H}), 3.23(\mathrm{~m}, 2 \mathrm{H}), 2.62$ $(\mathrm{m}, 2 \mathrm{H}), 2.09(\mathrm{~m}, 2 \mathrm{H}), 1.80(\mathrm{~s}, 3 \mathrm{H}) \mathrm{ppm} ;{ }^{13} \mathrm{C}$ NMR (100 MHz, DMSO-d $d_{6} \delta 200.02,167.78$, $164.01,160.45,150.60,148.35,139.85,135.44,130.28,122.95,116.97,36.87,27.89,22.33$, 10.45 ppm. MS (EI, $70 \mathrm{eV}): \mathrm{m} / z$ (\%) 323 (56.5) $\left[\mathrm{M}^{+}\right], 267$ (43.1), 201 (99.9), 123 (17.6), 95 (12.3). Anal. Calcd. for $\mathrm{C}_{17} \mathrm{H}_{13} \mathrm{~N}_{3} \mathrm{O}_{4}$ : C 63.16, H 4.05, N 13.00. Found: C 62.94, H 3.76, N $12.91 \%$.

4-(4-Chlorophenyl)-3,7,7-trimethyl-7,8-dihydroisoxazolo[5,4-b]quinolin-5(6H)-one (14k). Colorless solid, mp 240-241 ${ }^{\circ} \mathrm{C} .{ }^{1} \mathrm{H}$ NMR (200 MHz, DMSO-d $\left.d_{6}\right) \delta 7.50-7.30(\mathrm{~m}, 4 \mathrm{H}), 3.17$ (s, 2H), $2.52(\mathrm{~s}, 2 \mathrm{H}), 1.80(\mathrm{~s}, 3 \mathrm{H}), 1.04(\mathrm{~s}, 6 \mathrm{H}) \mathrm{ppm} ;{ }^{13} \mathrm{C}$ NMR (100 MHz, DMSO-d 6$) \delta 199.65$, $165.67,163.80,158.94,151.78,148.12$, 138.90, 129.52, 128.92, 128.63, 119.28, 52.95, 45.14,

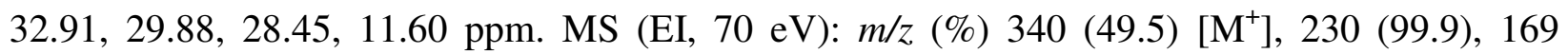
(52.3), 113 (77.4), 96 (17.8). Anal. Calcd. for $\mathrm{C}_{19} \mathrm{H}_{17} \mathrm{ClN}_{2} \mathrm{O}_{2}$ : C 66.96, H 5.03, N 8.22. Found: C $66.74, \mathrm{H} 4.73, \mathrm{~N} 8.10 \%$.

4-(4-Bromophenyl)-3,7,7-trimethyl-7,8-tetrahydroisoxazolo[5,4-b]quinolin-5(6H)-one (14l). Colorless solid, mp 256-257 ${ }^{\circ} \mathrm{C} .{ }^{1} \mathrm{H}$ NMR (200 MHz, DMSO-d $\left.d_{6}\right) \delta$ 7.63-7.26 (m, 4H), 3.17 (s, 2H), $2.51(\mathrm{~s}, 2 \mathrm{H}), 1.81(\mathrm{~s}, 3 \mathrm{H}), 1.05(\mathrm{~s}, 6 \mathrm{H}) \mathrm{ppm} ;{ }^{13} \mathrm{C}$ NMR (100 MHz, DMSO-d 6$) \delta 199.56$, $166.80,163.88,160.95,146.44,140.87,131.40,130.24,127.88,125.76,119.52,50.98,41.88$,

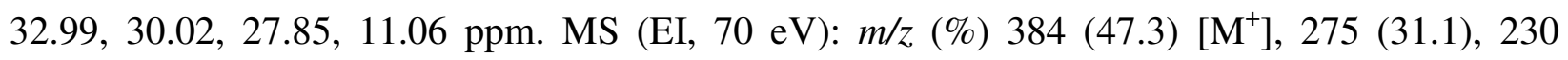
(99.9), 156 (27.1), 123 (18.5). Anal. Calcd. for $\mathrm{C}_{19} \mathrm{H}_{17} \mathrm{BrN}_{2} \mathrm{O}_{2}$ : C 59.24, H 4.45, N 7.27. Found: C 59.05, H 4.14, N 7.18\%.

3,7,7-Trimethyl-(4-methylphenyl)-7,8-tetrahydroisoxazolo[5,4-b]quinolin-5(6H)-one (14m). Colorless solid, mp 218-219 ${ }^{\circ} \mathrm{C} .{ }^{1} \mathrm{H}$ NMR (200 MHz, DMSO- $\left.d_{6}\right)$ 7.25-7.13 (m, 4H), 3.16 (s, 2H), $2.50(\mathrm{~s}, 2 \mathrm{H}), 2.38(\mathrm{~s}, 3 \mathrm{H}), 1.78(\mathrm{~s}, 3 \mathrm{H}), 1.04(\mathrm{~s}, 3 \mathrm{H}) \mathrm{ppm} ;{ }^{13} \mathrm{C}$ NMR (100 MHz, DMSO- $\left.d_{6}\right)$ $\delta 199.89,166.78,164.02,160.78,148.19,140.45,139.00,133.73,131.22,125.52,119.01,51.89$,

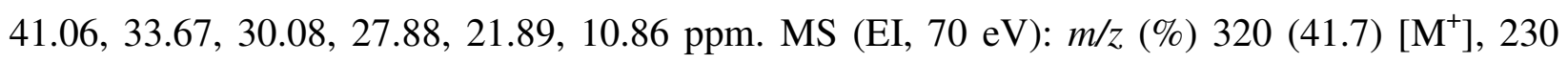
(99.9), 223 (19.9), 123 (19.3), 92(24.1). Anal. Calcd. for $\mathrm{C}_{20} \mathrm{H}_{20} \mathrm{~N}_{2} \mathrm{O}_{2}$ : C 74.98, H 6.29, N 8.74. Found: C 74.77, H 6.01, N 8.64\%.

Methyl-4-(3,7,7-trimethyl-5-oxo-5,6,7,8-tetrahydroisoxazolo[5,4-b]quinolin-4-yl)benzoate (14p). Colorless solid, mp 164-165 ${ }^{\circ} \mathrm{C} .{ }^{1} \mathrm{H}$ NMR (200 MHz, DMSO- $\left.d_{6}\right) \delta$ 8.04-7.42 (m, 4H), $3.89(\mathrm{~s}, 3 \mathrm{H}), 3.18(\mathrm{~s}, 2 \mathrm{H}), 2.52(\mathrm{~s}, 2 \mathrm{H}), 1.74(\mathrm{~s}, 3 \mathrm{H}), 1.05(\mathrm{~s}, 6 \mathrm{H}) \mathrm{ppm} ;{ }^{13} \mathrm{C} \mathrm{NMR}(100 \mathrm{MHz}$, DMSO- $\left.d_{6}\right) \delta 198.99,167.07,165.25,163.56,160.07,142.89,140.22,138.76,132.10,121.65$, 118.12, 51.95, 50.88, 41.87, 30.93, 28.72, 26.99, 10.14 ppm. MS (EI, $70 \mathrm{eV}): \mathrm{m} / z$ (\%) 364 (17.9) $\left[\mathrm{M}^{+}\right], 230$ (99.9), 169 (51.1), 135 (68.5). Anal. Calcd. for $\mathrm{C}_{21} \mathrm{H}_{20} \mathrm{~N}_{2} \mathrm{O}_{4}$ : C 69.22, H 5.53, N 7.69. Found: C 69.03, H 5.22, N 7.60\%.

4-(4-Bromophenyl)-7-(4-chlorophenyl)-3-methyl-7,8-dihydroisoxazolo[5,4-b]quinolin5(6H)-one (14u). Colorless solid, mp 188-189 ${ }^{\circ} \mathrm{C} .{ }^{1} \mathrm{H}$ NMR $\left(200 \mathrm{MHz}, \mathrm{DMSO}-d_{6}\right) \delta$ 7.68-7.28 
$(\mathrm{m}, 8 \mathrm{H}), 3.60(\mathrm{~m}, 2 \mathrm{H}), 3.27(\mathrm{~m}, 1 \mathrm{H}), 3.01(\mathrm{~m}, 1 \mathrm{H}), 2.71(\mathrm{~m}, 1 \mathrm{H}), 1.83(\mathrm{~s}, 3 \mathrm{H}) \mathrm{ppm} ;{ }^{13} \mathrm{C}$ NMR $\left(100 \mathrm{MHz}, \mathrm{DMSO}-d_{6}\right) \delta 198.11,166.95,164.12,160.76,146.54,142.56,138.89,136.26,133.45$, 132.11, 129.86, 127.44, 124.25, 122.19, 119.50, 47.01, 40.14, 37.31, 11.34 ppm. MS (EI, 70 $\mathrm{eV}): m / z(\%) 468$ (9.8) $\left[\mathrm{M}^{+}\right], 357$ (81.2), 311 (99.9), 201 (41.2), 156 (17.8), 113 (14.3). Anal. Calcd. for $\mathrm{C}_{23} \mathrm{H}_{16} \mathrm{BrClN}_{2} \mathrm{O}_{2}$ : C 59.06, H 3.45, N 5.99. Found: C 58.84, H 3.17, N 5.88\%.

7-(4-Chlorophenyl)-4-(4-methoxylphenyl)-3-methyl-7,8-dihydroisoxazolo[5,4-b]quinolin-

5(6H)-one (14w). Colorless solid; mp 168-169 ${ }^{\circ} \mathrm{C} .{ }^{1} \mathrm{H}$ NMR $\left(200 \mathrm{MHz}, \mathrm{DMSO}-d_{6}\right) \delta$ 7.75-7.04 $(\mathrm{m}, 8 \mathrm{H}), 3.65(\mathrm{~s}, 3 \mathrm{H}), 3.60(\mathrm{~m}, 2 \mathrm{H}), 3.27(\mathrm{~m}, 1 \mathrm{H}), 3.01(\mathrm{~m}, 1 \mathrm{H}), 2.71(\mathrm{~m}, 1 \mathrm{H}), 1.83(\mathrm{~s}, 3 \mathrm{H}) \mathrm{ppm}$; ${ }^{13} \mathrm{C}$ NMR (100 MHz, DMSO-d $\left.d_{6}\right) \delta 199.89,167.99,164.59,160.05,158.98,151.19,144.45$, $141.22,138.30,131.98,130.31,128.78,126.84,123.87,119.58,56.32,46.18,42.35,37.16$, 10.92 ppm. MS (EI, $70 \mathrm{eV}): \mathrm{m} / z(\%) 418$ (49.8) $\left[\mathrm{M}^{+}\right], 360$ (20.9), 280 (99.9), 279 (30.9), 140 (18.6), 125 (18.6), 102 (14.6). Anal. Calcd. for $\mathrm{C}_{24} \mathrm{H}_{19} \mathrm{ClN}_{2} \mathrm{O}_{3}: \mathrm{C}$ 68.82, H 4.57, N 6.69. Found: C 68.64, H 4.25, N 6.61\%.

General procedure for synthesis of 15a-f. A mixture of $\mathbf{4 c}, \mathbf{h}, \mathbf{n}, \mathbf{v}, \mathbf{z}(1 \mathrm{mmol})$, ethyl or benzyl bromide $(1 \mathrm{mmol})$ and $\mathrm{K}_{2} \mathrm{CO}_{3}(3 \mathrm{mmol})$ was heated in DMF $(0.3 \mathrm{~mL})$ for $2 \mathrm{~h}$. The reaction mixture was poured into water and the precipitate formed was filtered off, recrystallized from ethanol and dried in air.

4-(4-Bromophenyl)-9-ethyl-3-methyl-4,7,8,9-tetrahydroisoxazolo[5,4-b]quinolin-5(6H)-one (15a). Colorless solid, mp 235-236 ${ }^{\circ} \mathrm{C}$. ${ }^{1} \mathrm{H}$ NMR (200 MHz, DMSO- $\left.d_{6}\right) \delta 7.42-7.10(\mathrm{~m}, 4 \mathrm{H})$, $5.01(\mathrm{~s}, 1 \mathrm{H}), 3.85(\mathrm{q}, J 7.0 \mathrm{~Hz}, 2 \mathrm{H}), 2.74(\mathrm{~m}, 2 \mathrm{H}), 2.18(\mathrm{~m}, 2 \mathrm{H}), 1.87(\mathrm{~s}, 3 \mathrm{H}), 1.83(\mathrm{~m}, 2 \mathrm{H}), 1.24$ $(\mathrm{t}, J 7.0 \mathrm{~Hz}, 3 \mathrm{H}) \mathrm{ppm} ;{ }^{13} \mathrm{C}$ NMR $\left(100 \mathrm{MHz}, \mathrm{DMSO}-d_{6}\right) \delta 195.04,158.60,156.80,151.13$, $145.88,130.11,128.19,119.51,111.12,95.75,42.15,37.34,35.83,27.64,21.35,14.29,10.29$

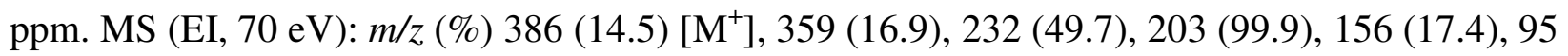
(37.8). Anal. Calcd. for $\mathrm{C}_{19} \mathrm{H}_{19} \mathrm{BrN}_{2} \mathrm{O}_{2}$ : C 58.93, $\mathrm{H}$ 4.95, $\mathrm{N}$ 7.23. Found: C 58.71, H 4.66, N $7.09 \%$.

\section{9-Benzyl-4-[4-(dimethylamino)phenyl]-3-methyl-4,7,8,9-tetrahydroisoxazolo[5,4-b]quino-} lin-5(6H)-one (15b). Colorless solid, mp 228-229 ${ }^{\circ} \mathrm{C} .{ }^{1} \mathrm{H}$ NMR $\left(200 \mathrm{MHz}, \mathrm{DMSO}-d_{6}\right) \delta$ 7.34$6.60(\mathrm{~m}, 9 \mathrm{H}), 5.11(\mathrm{~s}, 2 \mathrm{H}), 4.94(\mathrm{~s}, 1 \mathrm{H}), 2.83(\mathrm{~m}, 2 \mathrm{H}), 2.80(\mathrm{~s}, 6 \mathrm{H}), 2.15(\mathrm{~m}, 2 \mathrm{H}), 1.89(\mathrm{~s}, 3 \mathrm{H})$, $1.81(\mathrm{~m}, 2 \mathrm{H}) \mathrm{ppm} ;{ }^{13} \mathrm{C}$ NMR $\left(100 \mathrm{MHz}, \mathrm{DMSO}-d_{6}\right) \delta 194.42,158.92,158.35,151.99,148.77$, 137.13, 135.84, 128.76, 127.93, 127.09, 112.31, 111.63, 96.06, 48.76, 40.34, 36.98, 34.33, 27.54, 20.12, 9.89 ppm. MS (EI, $70 \mathrm{eV}): \mathrm{m} / z$ (\%) 413 (9.2) $\left[\mathrm{M}^{+}\right], 391$ (19.8), 343 (46.9), 341 (99.9), 153 (10.3), 138 (11.6), 125 (15.8), 103 (17.7), 91 (19.3), 77 (18.1). Anal. Calcd. for $\mathrm{C}_{26} \mathrm{H}_{27} \mathrm{~N}_{3} \mathrm{O}_{2}$ : C 75.52, H 6.58, N 10.16. Found: C 75.34, H 6.26, N 10.03\%.

9-Benzyl-4-(4-methoxyphenyl)-3,7,7-trimethyl-4,7,8,9-tetrahydroisoxazolo[5,4-b]quinolin5(6H)-one (15c). Colorless solid, mp 243-244 ${ }^{\circ} \mathrm{C}$. ${ }^{1} \mathrm{H}$ NMR $\left(200 \mathrm{MHz}\right.$, DMSO- $\left.d_{6}\right) \delta$ 7.42-6.75 $(\mathrm{m}, 9 \mathrm{H}), 5.12(\mathrm{~s}, 2 \mathrm{H}), 4.99(\mathrm{~s}, 1 \mathrm{H}), 3.68(\mathrm{~s}, 3 \mathrm{H}), 2.65-2.45(\mathrm{dd}, 2 \mathrm{H}), 2.16-1.99(\mathrm{dd}, 2 \mathrm{H}), 1.87$ (s, $3 \mathrm{H}), 0.93$ (s, 3H), 0.83 (s, 3H) ppm; ${ }^{13} \mathrm{C}$ NMR (100 MHz, DMSO-d $) \delta 195.16,159.76,158.93$, 153.32 , 150.44, 139.60, 137.23, 129.10, 128.19, 127.65, 113.98, 112.13, 96.53, 55.76, 52.31, 49.37, 37.81, 35.23, 32.56, 27.91, 21.59, 10.85 ppm. MS (EI, $70 \mathrm{eV}): \mathrm{m} / z(\%) 429(12.3)\left[\mathrm{M}^{+}\right]$, 
339 (99.9), 323 (14.5), 231 (19.6), 170 (38.4), 108 (41.3). Anal. Calcd. for $\mathrm{C}_{27} \mathrm{H}_{28} \mathrm{~N}_{2} \mathrm{O}_{3}$ : C 75.68, H 6.59, N 6.54. Found: C 75.50, H 6.27, N 6.42\%.

\section{7-(4-Chlorophenyl)-9-ethyl-3-methyl-4-(4-methylphenyl)-4,7,8,9-tetrahydroisoxazolo-}

[5,4-b]quinolin-5(6H)-one (15d). Colorless solid, mp 139-140 ${ }^{\circ} \mathrm{C}$. ${ }^{1} \mathrm{H}$ NMR $(200 \mathrm{MHz}$, DMSO$\left.d_{6}\right) \delta 7.33-6.97(\mathrm{~m}, 8 \mathrm{H}), 4.95(\mathrm{~s}, 1 \mathrm{H}), 3.88(\mathrm{~m}, 2 \mathrm{H}), 3.46(\mathrm{~m}, 1 \mathrm{H}), 3.00(\mathrm{~m}, 2 \mathrm{H}), 2.40(\mathrm{~m}, 2 \mathrm{H})$, $2.20(\mathrm{~s}, 3 \mathrm{H}), 1.87(\mathrm{~s}, 3 \mathrm{H}), 1.22(\mathrm{~m}, 3 \mathrm{H}) \mathrm{ppm} ;{ }^{13} \mathrm{C}$ NMR (100 MHz, DMSO- $\left.d_{6}\right) \delta 193.12,159.40$, $158.15,155.25,151.30,147.20,143.95,142.67,135.37,131.67,129.38,127.94,111.25,96.55$, 44.16, 41.25, 38.38, 36.01, 34.38, 21.08, 14.29, 10.34 ppm. MS (EI, $70 \mathrm{eV}): \mathrm{m} / z$ (\%) 432 (33.1) $\left[\mathrm{M}^{+}\right], 431$ (40.9), 343 (50.8), 341 (99.9), 225 (13.1), 174 (16.3), 153 (13.7), 127 (15.8), 102 (18.0). Anal. Calcd. for $\mathrm{C}_{26} \mathrm{H}_{25} \mathrm{~N}_{2} \mathrm{O}_{2}$ : C 72.13, H 5.82, N 6.47. Found: C 71.91, H 5.53, N 6.35\%.

\section{9-Benzyl-7-(4-chlorophenyl)-3-methyl-4-(4-methylphenyl)-4,7,8,9-tetrahydroisoxazolo-} [5,4-b]quinolin-5(6H)-one (15e). Colorless solid, mp 225-226 ${ }^{\circ} \mathrm{C} .{ }^{1} \mathrm{H}$ NMR (200 MHz, DMSO$\left.d_{6}\right) \delta 7.38-6.95(\mathrm{~m}, 13 \mathrm{H}), 5.13(\mathrm{~s}, 2 \mathrm{H}), 5.00(\mathrm{~s}, 1 \mathrm{H}), 3.46(\mathrm{~m}, 1 \mathrm{H}), 2.98(\mathrm{~m}, 2 \mathrm{H}), 2.54(\mathrm{~m}, 2 \mathrm{H})$, $2.20(\mathrm{~s}, 3 \mathrm{H}), 1.88(\mathrm{~s}, 3 \mathrm{H}) \mathrm{ppm} ;{ }^{13} \mathrm{C}$ NMR (100 MHz, DMSO- $\left.d_{6}\right) \delta$ 193.72, 159.42, 158.90, $155.35,147.18,143.90,142.54,138.18,135.17,132.23,129.18,127.04,126.93,125.16,124.88$, 110.18, 96.17, 47.65, 44.18, 38.42, 36.16, 34.25, 21.38, 10.85 ppm. MS (EI, 70 eV): m/z (\%) 494 (10.7) $\left[\mathrm{M}^{+}\right], 453$ (22.3), 405 (46.6), 403 (97.3), 127 (11.2), 115 (14.8), 92 (44.3), 91 (99.9), 77 (12.1). Anal. Calcd. for $\mathrm{C}_{31} \mathrm{H}_{27} \mathrm{ClN}_{2} \mathrm{O}_{2}$ : C 75.22, H 5.50, N 5.66. Found: C 75.01, H 5.19, N $5.56 \%$.

9-Benzyl-7-(4-chlorophenyl)-4-[4-(dimethylamino)phenyl]-3-methyl-4,7,8,9-tetrahydroisoxazolo[5,4-b]quinolin-5(6H)-one (15f). Colorless solid, mp 152-153 ${ }^{\circ} \mathrm{C}$. ${ }^{1} \mathrm{H}$ NMR (200 MHz, DMSO-d $)_{6} \delta 7.38-6.49(\mathrm{~m}, 13 \mathrm{H}), 5.13(\mathrm{~s}, 2 \mathrm{H}), 4.92(\mathrm{~s}, 1 \mathrm{H}), 3.54(\mathrm{~m}, 1 \mathrm{H}), 2.97(\mathrm{~m}, 2 \mathrm{H}), 2.81(\mathrm{~s}$, $6 \mathrm{H}), 2.51(\mathrm{~m}, 2 \mathrm{H}), 1.89(\mathrm{~s}, 3 \mathrm{H}) \mathrm{ppm} ;{ }^{13} \mathrm{C}$ NMR (100 MHz, DMSO-d $\left.d_{6}\right) \delta 194.87,161.12,155.90$, $150.35,147.18,143.90,138.18,135.17,130.23,129.18,127.04,126.93,125.66,114.98,109.18$, 95.88, 47.15, 44.20, 41.95, 38.28, 36.22, 33.28, 10.85 ppm. MS (EI, $70 \mathrm{eV}): \mathrm{m} / z$ (\%) 523 (17.4) $\left[\mathrm{M}^{+}\right], 482$ (33.8), 391 (15.7), 91 (99.9), 65 (18.0). Anal. Calcd. for $\mathrm{C}_{32} \mathrm{H}_{30} \mathrm{ClN}_{3} \mathrm{O}_{2}: \mathrm{C} 73.34, \mathrm{H}$ 5.77, N 8.02. Found: C 73.12, H 5.48, N 7.91\%.

General procedure for synthesis of 13a,b. A mixture of 5-amino-3-methylisoxazole 1 (1 mmol), aldehydes 9a,d ( $1 \mathrm{mmol})$, cyclic $\beta$-di-ketones $3 \mathbf{b}(1 \mathrm{mmol})$ and $\mathrm{Et}_{3} \mathrm{~N}(0.1 \mathrm{mmol})$ in DMF $(2 \mathrm{~mL})$ was heated to reflux for $10 \mathrm{~min}$ in a roundbottom flask equipped with a condenser. Then after cooling ethanol $(10 \mathrm{~mL})$ was added and the precipitate formed was filtered off to give the solid compounds.

9-(2-Hydroxy-4,4-dimethyl-6-oxocyclohex-1-enyl)-3,3-dimethyl-3,4-dihydro-2H-xanthen1(9H)-one (13a). Colorless solid, mp 209-210 ${ }^{\circ} \mathrm{C} .{ }^{1} \mathrm{H}$ NMR (200 MHz, DMSO- $\left.d_{6}\right) \delta 10.29$ (s, $1 \mathrm{OH}), 7.00-6.82(\mathrm{~m}, 4 \mathrm{H}), 5.02(\mathrm{~s}, 1 \mathrm{H}), 2.50-2.20(\mathrm{~m}, 4 \mathrm{H}), 2.09-1.94(\mathrm{~m}, 4 \mathrm{H}), 1.02(\mathrm{~s}, 3 \mathrm{H}), 0.96$ $(\mathrm{s}, 3 \mathrm{H}), 0.87(\mathrm{~s}, 6 \mathrm{H}) \mathrm{ppm}$; Anal. Calcd. for $\mathrm{C}_{23} \mathrm{H}_{26} \mathrm{O}_{4}: \mathrm{C} 75.38, \mathrm{H}$ 7.15. Found: C 75.12, $\mathrm{H}$ $6.86 \%$.

9-(2-Hydroxy-4,4-dimethyl-6-oxocyclohex-1-enyl)-5-methoxy-3,3-dimethyl-3,4-dihydro-2Hxanthen-1(9H)-one (13b). Colorless solid, mp 235-236 ${ }^{\circ} \mathrm{C} .{ }^{1} \mathrm{H}$ NMR (200 MHz, DMSO- $\left.d_{6}\right) \delta$ 
10.29 (s, 1OH), 6.92-6.49 (m, 3H), $5.01(\mathrm{~s}, 1 \mathrm{H}), 3.76(\mathrm{~s}, 3 \mathrm{H}), 2.54-2.27(\mathrm{~m}, 4 \mathrm{H}), 2.14-1.99$ (m, $2 \mathrm{H}), 1.02(\mathrm{~s}, 3 \mathrm{H}), 0.96(\mathrm{~s}, 3 \mathrm{H}), 0.86(\mathrm{~s}, 6 \mathrm{H}) \mathrm{ppm}$; Anal. Calcd. for $\mathrm{C}_{24} \mathrm{H}_{28} \mathrm{O}_{5}$ : C 72.70, $\mathrm{H} 7.12$. Found: C 72.46, H $6.84 \%$.

\section{Acknowledgements}

VAC was supported by DAAD scholarship for scientists. Authors thank to Dr. V. Polovinko and Dr. A. Tverdokhlebov (Enamine Ltd, Ukraine) for recording some ${ }^{13} \mathrm{C}$ NMR spectra.

\section{References}

1. Shin, K. D.; Lee, M. Y.; Shin, D. S.; Lee, S.; Son, K. H.; Koh, S.; Paik, Y. K.; Kwon, B. M.; Han, D. C. J. Biol. Chem. 2005, 280, 41439.

http://dx.doi.org/10.1074/jbc.M507209200

PMid:16234246

2. Demers, J.; Hageman, W.; Johnson, S.; Klaubert, D.; Look, R.; Moore, J. Bioorg. Med. Chem. Lett. 1994, 4, 2451. http://dx.doi.org/10.1016/S0960-894X(01)80408-X

3. Simoni, D.; Roberti, M.; Paolo, I. F.; Rondanin, R.; Baruchello, R.; Malagutti, C.; Mazzali, A.; Rossi, M.; Grimaudo, S.; Capone, F.; Dusonchet, L.; Meli, M.; Raimondi, M. V.; Landino, M.; D'Alessandro, N.; Tolomeo, M.; Arindam, D.; Lu, S.; Benbrook, D. M. J. Med. Chem. 2001, 44, 2308. http://dx.doi.org/10.1021/jm0010320

4. Rowley, M.; Broughton, H. B.; Collins, I.; Baker, R.; Emms, F.; Marwood, R.; Patel, S.; Patel, S.; Ragan, C. I.; Freedman, S. B.; Leeson, P. D. J. Med. Chem. 1996, 39, 1943. http://dx.doi.org/10.1021/jm960072u PMid:8642551

5. Wittenberger, S. J. J. Org. Chem. 1996, 61, 356. http://dx.doi.org/10.1021/jo9509723

6. Dannhardt, G.; Dominiak, P.; Laufer, S. Arznei-Forschung. 1993, 43, 441. PMid:8494574

7. Tatee, T.; Narita, K.; Kurashige, S.; Ito, S; Miyazaki, H.; Yamanaka, H.; Mizugaki, M.; Sakamoto, T.; Fukuda, H. Chem. Pharm. Bull. 1987, 35, 3676. http://dx.doi.org/10.1248/cpb.35.3676

8. Shi, F.; Zeng, X.-N.; Cao, X.-D.; Zhang, S.; Jiang, B.; Zheng, W. F.; Tu, S.-J. Bioorg. Med. Chem. Lett. 2012, 22, 743.

http://dx.doi.org/10.1016/j.bmcl.2011.09.081

PMid:22137847 
9. Kamal, A.; Suresh, P.; Mallareddy, A.; Kumar, B. A.; Reddy, P. V.; Raju, P.; Tamboli, J. R.; Shaik, T. B.; Jain, N.; Kalivendi, S. V. Bioorg. Med. Chem. 2011, 19, 2349.

http://dx.doi.org/10.1016/j.bmc.2011.02.020

PMid:21402478

10. Tu, S.-J.; Zhang, X.-H.; Han, Z.-G.; Cao, X.-D.; Wu, S.-S.; Yan, S.; Hao, W.-J.; Zhang, G.; Ma, N. J. Comb. Chem. 2009, 11, 428.

http://dx.doi.org/10.1021/cc800212v

PMid:19364093

11. Ma, N.; Jiang, B.; Zhang, G.; Tu, S.-J.; Weverb, W.; Li, G. Green Chem. 2010, 12, 1357. http://dx.doi.org/10.1039/c0gc00073f

12. Jiang, B.; Zhang, G.; Ma, N.; Shi, F.; Tu, S.-J.; Kaurb, P.; Li, G. Org. Biomol. Chem. 2011, 9, 3834.

http://dx.doi.org/10.1039/c1ob05034f

PMid:21445382

13. Chebanov, V. A.; Gura, K. A; Desenko S. M. Top. Heterocycl. Chem. 2010, 23, 41. http://dx.doi.org/10.1007/7081_2009_21

14. Chebanov, V. A.; Desenko, S. M. Chem. Heterocycl. Comp. 2012, 48, 566. http://dx.doi.org/10.1007/s10593-012-1030-2

15. Sedash, Yu. V.; Gorobets, N. Yu.; Chebanov, V. A.; Konovalova, I. S.; Shishkin, O. V.; Desenko, S. M. RSC Advances 2012, 2, 6719.

http://dx.doi.org/10.1039/c2ra20195j

16. Muravyova, E. A.; Desenko, S. M.; Rudenko, R. V.; Shishkina, S. V.; Shishkin, O. V.; Sen'ko, Y. V.; Vashchenko, E. V.; Chebanov, V. A. Tetrahedron 2011, 67, 9389. http://dx.doi.org/10.1016/j.tet.2011.09.138

17. Sakhno, Y. I.; Shishkina, S. V.; Shishkin, O. V.; Musatov, V. I.; Vashchenko, E. V.; Desenko, S. M.; Chebanov, V. A. Mol. Divers. 2010, 9, 523.

http://dx.doi.org/10.1007/s11030-010-9226-9

PMid:20229319

18. Muravyova, E. A.; Shishkina, S. V.; Musatov, V. I.; Knyazeva, I. V.; Shishkin, O. V.; Desenko, S. M.; Chebanov, V. A. Synthesis 2009, 1375.

19. Sakhno, Y. I.; Desenko, S. M.; Shishkina, S. V.; Shishkin, O. V.; Sysoyev, D. O.; Groth, U.; Kappe, C. O.; Chebanov, V. A. Tetrahedron 2008, 64, 11041. http://dx.doi.org/10.1016/j.tet.2008.09.089

20. Chebanov, V. A.; Saraev, V. E.; Desenko, S. M.; Chernenko, V. N.; Knyazeva, I. V.; Groth, U.; Glasnov, T. N.; Kappe, C. O. J. Org. Chem. 2008, 73, 5110.

http://dx.doi.org/10.1021/jo800825c

PMid:18512991

21. Chebanov, V. A.; Sakhno, Y. I.; Desenko, S. M.; Chernenko, V. N.; Musatov, V. I.; Shishkina, S. V.; Shishkin, O. V.; Kappe, C. O. Tetrahedron 2007, 63, 1229. http://dx.doi.org/10.1016/j.tet.2006.11.048 
22. Chebanov, V. A.; Saraev, V. E.; Shishkina, S. V.; Shishkin, O. V.; Musatov, V. I.; Desenko, S. M. Eur. J. Org. Chem. 2012, 5515. http://dx.doi.org/10.1002/ejoc.201200669

23. Gorobets, N. Yu.; Sedash, Yu. V.; Ostras, K. S.; Zaremba, O. V.; Shishkina, S. V.; Baumer, V. N.; Shishkin, O. V.; Kovalenko, S. N.; Desenko, S. M.; van der Eycken, E. Tetrahedron Lett. 2010, 51, 2095.

http://dx.doi.org/10.1016/j.tetlet.2010.02.045

24. Svělík, J.; Kettmann, V. Tetrahedron Lett. 2011, 52, 1062.

http://dx.doi.org/10.1016/j.tetlet.2010.12.051

25. Frolova, L. V.; Malik, I.; Uglinskii, P. Y.; Rogelj, S.; Kornienko, A.; Magedov, I. V. Tetrahedron Lett. 2011, 52, 6643.

http://dx.doi.org/10.1016/j.tetlet.2011.10.012

PMid:22162894, PMCid:3234110

26. Svělík, J.; Veizerová, L.; Mayer, T. U.; Catarinella, M. Bioorg. Med. Chem. Lett. 2010, 20, 4073.

http://dx.doi.org/10.1016/j.bmcl.2010.05.085

PMid:20542426 